Network Working Group

Request for Comments: 2741

Obsoletes: 2257

Category: Standards Track
M. Daniele Compaq Computer Corporation B. Wijnen T.J. Watson Research Center, IBM Corp. M. Ellison, Ed. Ellison Software Consulting, Inc. D. Francisco. Ed. Cisco Systems, Inc. January 2000

\title{
Agent Extensibility (Agentx) Protocol Version 1
}

Status of this Memo

This document specifies an Internet standards track protocol for the Internet community, and requests discussion and suggestions for improvements. Please refer to the current edition of the "Internet Official Protocol Standards" (STD 1) for the standardization state and status of this protocol. Distribution of this memo is unlimited.

Copyright Notice

Copyright (C) The Internet Society (2000). All Rights Reserved.

Abstract

This memo defines a standardized framework for extensible SNMP agents. It defines processing entities called master agents and subagents, a protocol (Agentx) used to communicate between them, and the elements of procedure by which the extensible agent processes SNMP protocol messages. This memo obsoletes RFC 2257.

Table of Contents

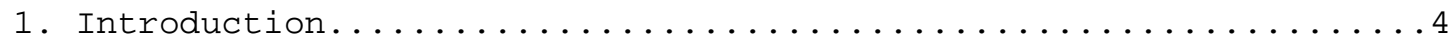

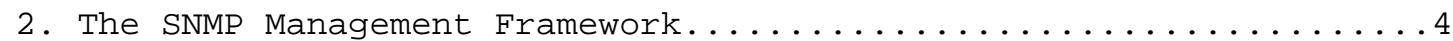

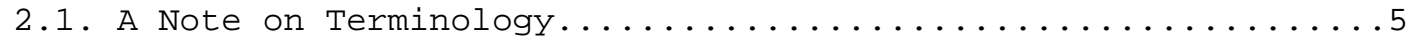

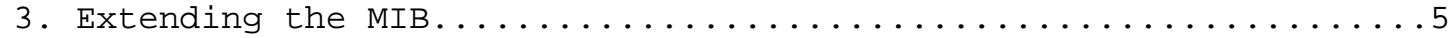

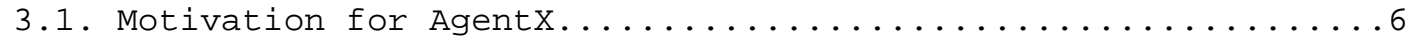

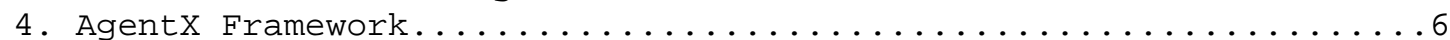

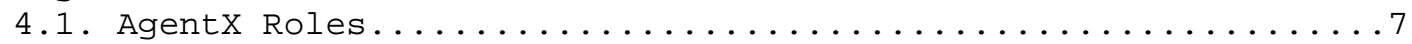

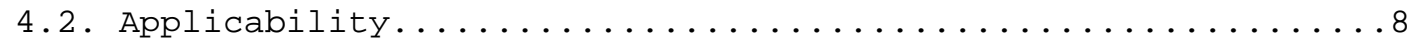

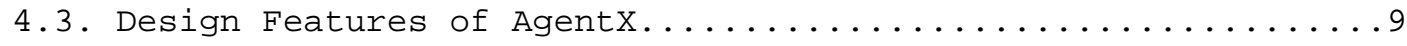

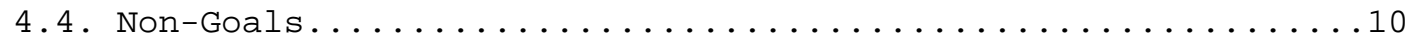




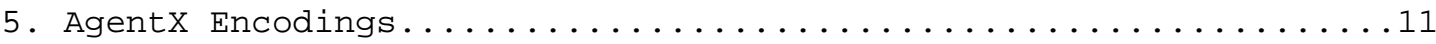

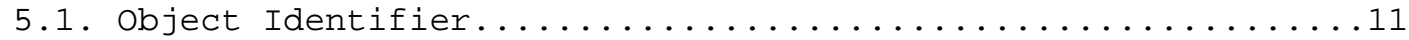

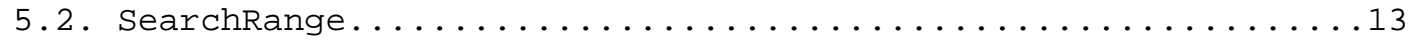

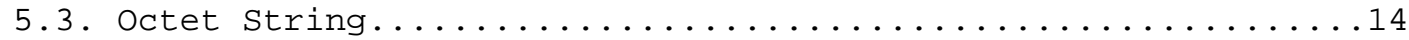

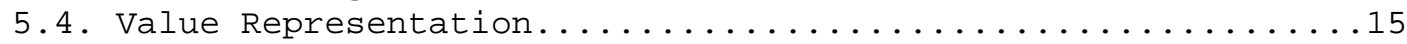

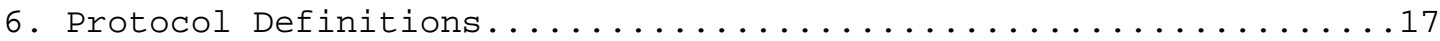

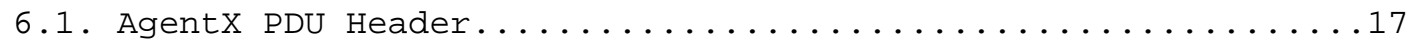

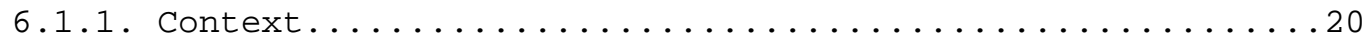

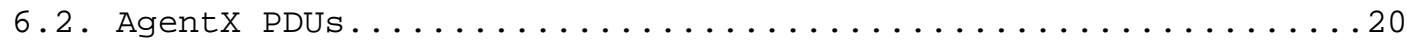

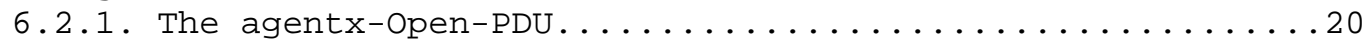

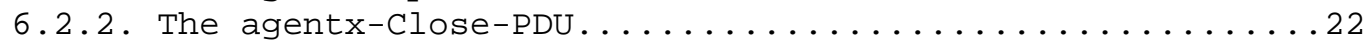

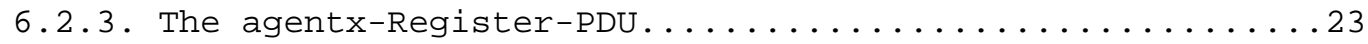

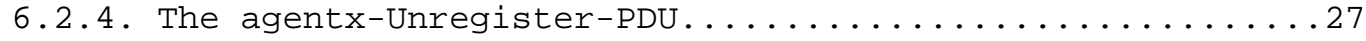

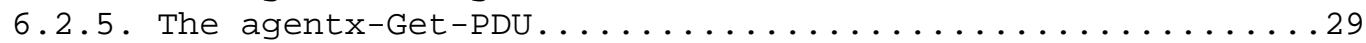

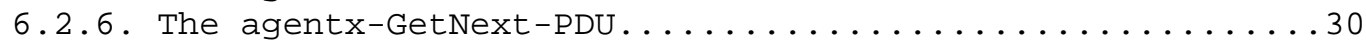

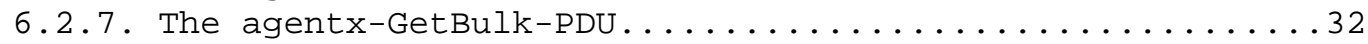

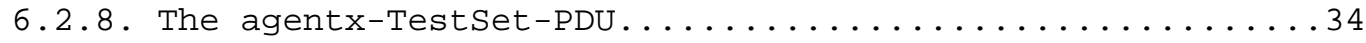

6.2.9. The agentx-Commitset, -Undoset, -Cleanupset PDUs......35

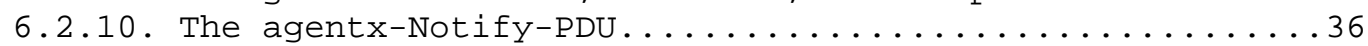

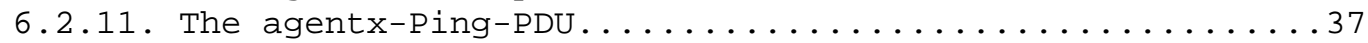

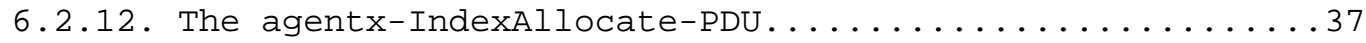

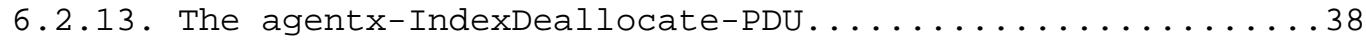

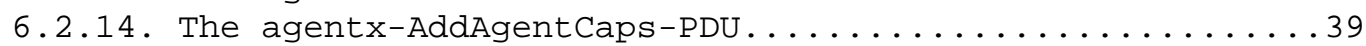

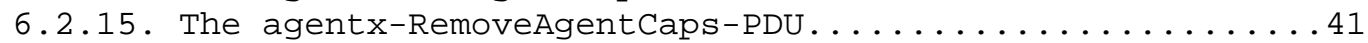

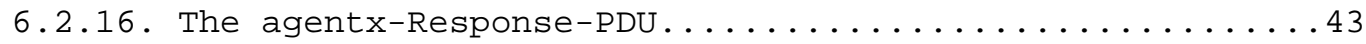

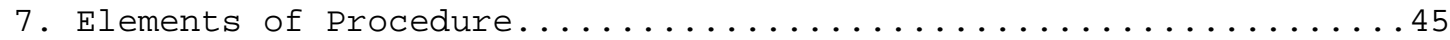

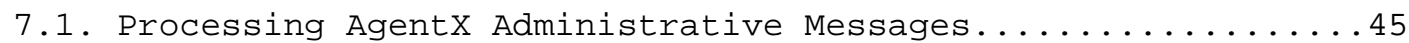

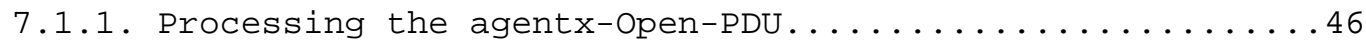

7.1.2. Processing the agentx-IndexAllocate-PDU.....................

7.1.3. Processing the agentx-IndexDeallocate-PDU...........49

7.1.4. Processing the agentx-Register-PDU.......................50

7.1.4.1. Handling Duplicate and Overlapping Subtrees......50

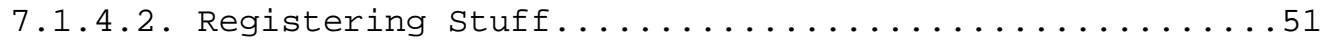

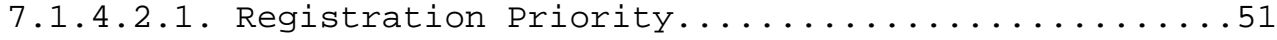

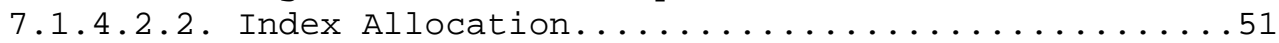

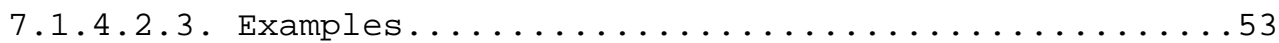

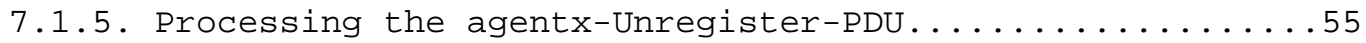

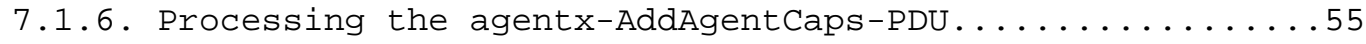

7.1.7. Processing the agentx-RemoveAgentCaps-PDU...........55

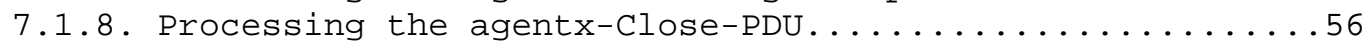

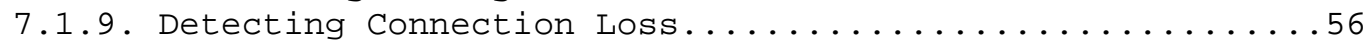

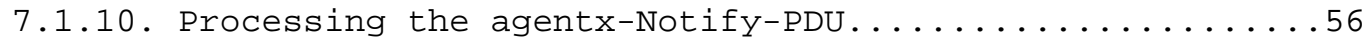

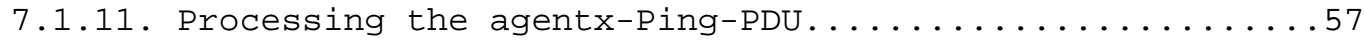

7.2. Processing Received SNMP Protocol Messages............58

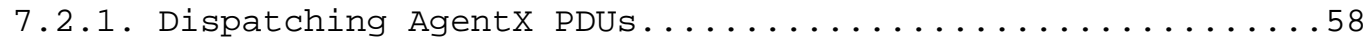

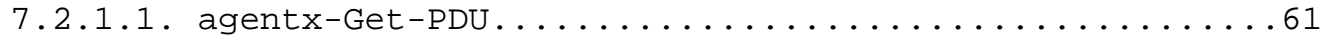

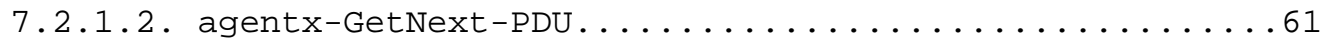

7.2 .1 .3 . agentx-GetBulk-PDU...................62 


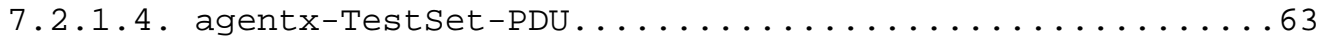

7.2 .1 .5$. Dispatch......................64

7.2 .2 . Subagent Processing......................64

7.2.3. Subagent Processing of agentx-Get, GetNext, GetBulk-PDUs65

7.2.3.1. Subagent Processing of the agentx-Get-PDU.......65

7.2.3.2. Subagent Processing of the agentx-GetNext-PDU....66

7.2.3.3. Subagent Processing of the agentx-GetBulk-PDU.....66

7.2.4. Subagent Processing of agentx-Testset, -Commitset,

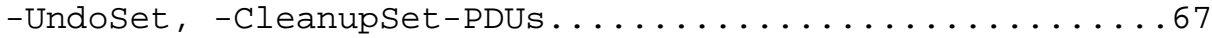

7.2.4.1. Subagent Processing of the agentx-TestSet-PDU.....68

7.2.4.2. Subagent Processing of the agentx-CommitSet-PDU....69

7.2.4.3. Subagent Processing of the agentx-UndoSet-PDU.....69

7.2.4.4. Subagent Processing of the agentx-CleanupSet-PDU...70

7.2.5. Master Agent Processing of AgentX Responses..........70

7.2.5.1. Common Processing of All AgentX Response PDUs......70

7.2.5.2. Processing of Responses to agentx-Get-PDUs........70

7.2.5.3. Processing of Responses to agentx-GetNext-PDU and agentx-GetBulk-PDU..............................

7.2.5.4. Processing of Responses to agentx-TestSet-PDUs....72

7.2.5.5. Processing of Responses to agentx-CommitSet-PDUs...73

7.2.5.6. Processing of Responses to agentx-UndoSet-PDUs.....74

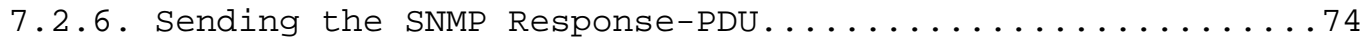

7.2 .7$. MIB Views......................... 74

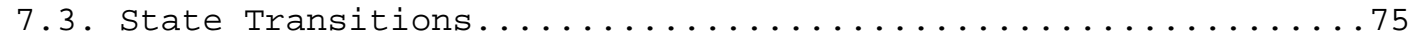

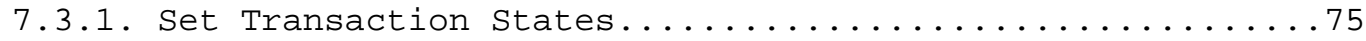

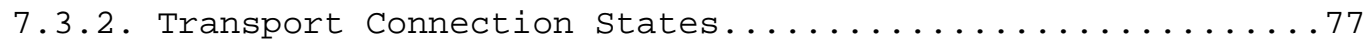

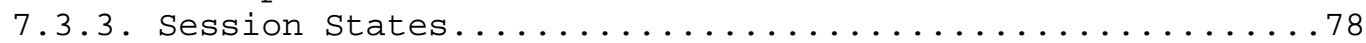

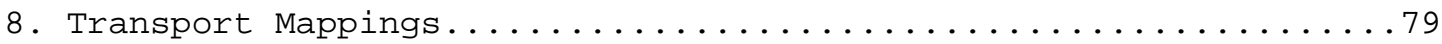

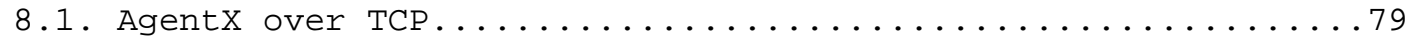

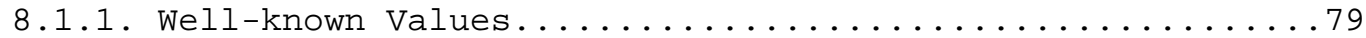

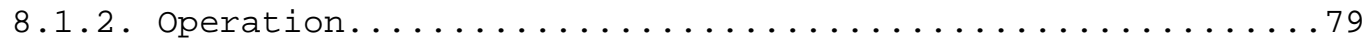

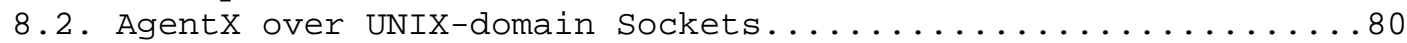

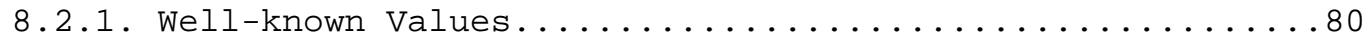

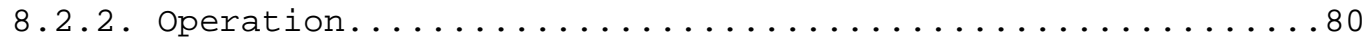

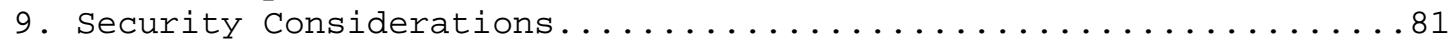

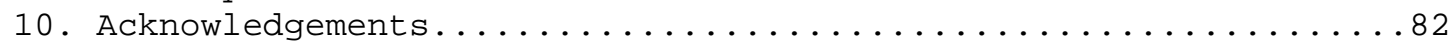

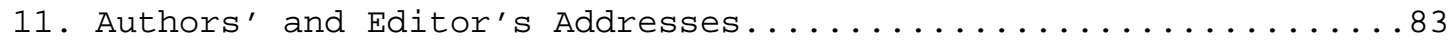

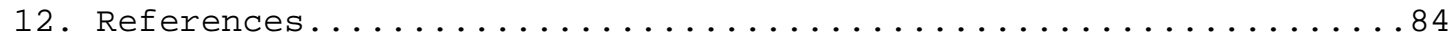

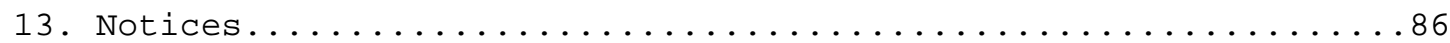

Appendix A. Changes relative to $\operatorname{RFC} 2257 \ldots \ldots \ldots \ldots \ldots \ldots$

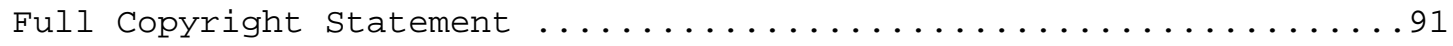




\section{Introduction}

This memo defines a standardized framework for extensible SNMP agents. It defines processing entities called master agents and subagents, a protocol (Agentx) used to communicate between them, and the elements of procedure by which the extensible agent processes SNMP protocol messages.

This memo obsoletes RFC 2257. It is worth noting that most of the changes are for the purpose of clarification. The only changes affecting Agentx protocol messages on the wire are:

- The agentx-Notify-PDU and agentx-Close-PDU now generate an agent $x-$ Response-PDU

- Three new error codes are available: parsefailed(266), requestDenied(267), and processingError(268)

Appendix A provides a detailed list of changes relative to RFC 2257 . The key words "MUST", "MUST NOT", "REQUIRED", "SHALL", "SHALL NOT", "SHOULD", "SHOULD NOT", "RECOMMENDED", "MAY", and "OPTIONAL" in this document are to be interpreted as described in [27].

2. The SNMP Management Framework

The SNMP Management Framework presently consists of five major components:

An overall architecture, described in RFC 2571 [1].

Mechanisms for describing and naming objects and events for the purpose of management. The first version of this structure of Management Information (SMI) is called SMIv1 and described in STD 16, $\mathrm{RFC} 1155$ [2], STD 16, RFC 1212 [3] and RFC 1215 [4]. The second version, called SMIv2, is described in STD 58, RFC 2578 [5], STD 58, RFC 2579 [6] and STD 58, RFC 2580 [7].

Message protocols for transferring management information. The first version of the SNMP message protocol is called SNMPVI and described in STD 15, RFC 1157 [8]. A second version of the SNMP message protocol, which is not an Internet standards track protocol, is called SNMPV2c and described in RFC 1901 [9] and RFC 1906 [10]. The third version of the message protocol is called SNMPv3 and described in RFC 1906 [10], RFC 2572 [11] and RFC 2574 [12].

Protocol operations for accessing management information. The first set of protocol operations and associated PDU formats is described in 
STD 15, RFC 1157 [8]. A second set of protocol operations and associated PDU formats is described in RFC 1905 [13].

A set of fundamental applications described in RFC 2573 [14] and the view-based access control mechanism described in RFC 2575 [15].

A more detailed introduction to the current SNMP Management Framework can be found in RFC 2570 [16].

Managed objects are accessed via a virtual information store, termed the Management Information Base or MIB. Objects in the MIB are defined using the mechanisms defined in the SMI.

\subsection{A Note on Terminology}

The term "variable" refers to an instance of a non-aggregate object type defined according to the conventions set forth in the SMIV2 (STD 58, RFC 2578, [5]) or the textual conventions based on the SMIV2 (STD 58, RFC 2579 [6]). The term "variable binding" normally refers to the pairing of the name of a variable and its associated value. However, if certain kinds of exceptional conditions occur during processing of a retrieval request, a variable binding will pair a name and an indication of that exception.

A variable-binding list is a simple list of variable bindings.

The name of a variable is an OBJECT IDENTIFIER, which is the concatenation of the OBJECT IDENTIFIER of the corresponding object type together with an OBJECT IDENTIFIER fragment identifying the instance. The OBJECT IDENTIFIER of the corresponding object-type is called the OBJECT IDENTIFIER prefix of the variable.

3. Extending the MIB

New MIB modules that extend the Internet-standard MIB are continuously being defined by various IETF working groups. It is also common for enterprises or individuals to create or extend enterprise-specific or experimental MIBs.

As a result, managed devices are frequently complex collections of manageable components that have been independently installed on a managed node. Each component provides instrumentation for the managed objects defined in the MIB module(s) it implements.

The SNMP framework does not describe how the set of managed objects supported by a particular agent may be changed dynamically . 


\subsection{Motivation for AgentX}

This very real need to dynamically extend the management objects within a node has given rise to a variety of "extensible agents", which typically comprise

- a "master" agent that is available on the standard transport address and that accepts SNMP protocol messages

- a set of "subagents" that each contain management instrumentation

- a protocol that operates between the master agent and subagents, permitting subagents to "connect" to the master agent, and the master agent to multiplex received SNMP protocol messages amongst the subagents.

- a set of tools to aid subagent development, and a runtime (API) environment that hides much of the protocol operation between a subagent and the master agent.

The wide deployment of extensible SNMP agents, coupled with the lack of Internet standards in this area, makes it difficult to field SNMP-manageable applications. A vendor may have to support several different subagent environments (APIs) in order to support different target platforms.

It can also become quite cumbersome to configure subagents and (possibly multiple) master agents on a particular managed node.

Specifying a standard protocol for agent extensibility (AgentX) provides the technical foundation required to solve both of these problems. Independently developed AgentX-capable master agents and subagents will be able to interoperate at the protocol level. Vendors can continue to differentiate their products in all other respects.

4. AgentX Framework

Within the SNMP framework, a managed node contains a processing entity, called an agent, which has access to management information.

Within the Agentx framework, an agent is further defined to consist of: 
- a single processing entity called the master agent, which sends and receives SNMP protocol messages in an agent role (as specified by the SNMP framework documents) but typically has little or no direct access to management information.

- zero or more processing entities called subagents, which are "shielded" from the SNMP protocol messages processed by the master agent, but which have access to management information.

The master and subagent entities communicate via Agentx protocol messages, as specified in this memo. Other interfaces (if any) on these entities, and their associated protocols, are outside the scope of this document. While some of the Agentx protocol messages appear similar in syntax and semantics to the SNMP, bear in mind that Agentx is not SNMP.

The internal operations of Agentx are invisible to an SNMP entity operating in a manager role. From a manager's point of view, an extensible agent behaves exactly as would a non-extensible (monolithic) agent that has access to the same management instrumentation.

This transparency to managers is a fundamental requirement of Agentx, and is what differentiates Agentx subagents from SNMP proxy agents.

\subsection{Agentx Roles}

An entity acting in a master agent role performs the following functions:

- Accepts Agentx session establishment requests from subagents.

- Accepts registration of MIB regions by subagents.

- Sends and accepts SNMP protocol messages on the agent's specified transport addresses.

- Implements the agent role Elements of Procedure specified for the administrative framework applicable to the SNMP protocol message, except where they specify performing management operations. (The application of MIB views, and the access control policy for the managed node, are implemented by the master agent.)

- Provides instrumentation for the MIB objects defined in RFC 1907 [17], and for any MIB objects relevant to any administrative framework it supports. 
- Sends and receives Agentx protocol messages to access management information, based on the current registry of MIB regions.

- Forwards notifications on behalf of subagents.

An entity acting in a subagent role performs the following functions:

- Initiates AgentX sessions with the master agent.

- Registers MIB regions with the master agent.

- Instantiates managed objects.

- Binds OIDs within its registered MIB regions to actual variables.

- Performs management operations on variables.

- Initiates notifications.

\subsection{Applicability}

It is intended that this memo specify the smallest amount of required behavior necessary to achieve the largest benefit, that is, to cover a very large number of possible MIB implementations and configurations with minimum complexity and low "cost of entry".

This section discusses several typical usage scenarios.

1) Subagents implement separate MIB modules -- for example, subagent ' $A$ ' implements "mib-2", subagent 'B' implements "host-resources".

It is anticipated that this will be the most common subagent configuration.

2) Subagents implement rows in a "simple table". A simple table is one in which row creation is not specified, and for which the MIB does not define an object that counts entries in the table. Examples of simple tables are rdbmsDbTable, udpTable, and hrSWRunTable.

This is the most commonly defined type of MIB table, and probably represents the next most typical configuration that Agentx would support. 
3) Subagents share MIBs along non-row partitions. Subagents register "chunks" of the MIB that represent multiple rows, due to the nature of the MIB's index structure. Examples include registering ipNetToMediaEntry.n, where $\mathrm{n}$ represents the ifIndex value for an interface implemented by the subagent, and tcpconnEntry.a.b.c.d, where a.b.c.d represents an IP address on an interface implemented by the subagent.

Agentx supports these three common configurations, and all permutations of them, completely. The consensus is that they comprise a very large majority of current and likely future uses of multi-vendor extensible agent configurations.

4) Subagents implement rows in tables that permit row creation, for example, the RMON historyControlTable. To implement row creation in such tables, at least one Agentx subagent must register at a point "higher" in the OID tree than an individual row (per AgentX's dispatching procedure).

5) Subagents implement rows in tables whose MIB also defines an object that counts entries in the table, for example the MIB-2 iftable (due to ifNumber). The subagent that implements such a counter object (like ifNumber) must go beyond Agentx to correctly implement it. This is an implementation issue (and most new MIB designs no longer include such objects).

Scenarios in these latter 2 categories were thought to occur somewhat rarely in configurations where subagents are independently implemented by different vendors. The focus of a standard protocol, however, must be in just those areas where multi-vendor interoperability must be assured.

Note that it would be inefficient (due to Agentx registration overhead) to share a table among Agentx subagents if the table contains very dynamic instances, and each subagent registers fully qualified instances. ipRouteTable could be an example of such a table in some environments.

\subsection{Design Features of Agentx}

The primary features of the design described in this memo are:

1) A general architectural division of labor between master agent and subagent: The master agent is MIB ignorant and SNMP omniscient, while the subagent is SNMP ignorant and MIB omniscient (for the MIB variables it instantiates). That is, master agents, exclusively, are concerned with SNMP protocol operations and the translations to and from Agentx protocol operations needed to 
carry them out; subagents are exclusively concerned with management instrumentation; and neither should intrude on the other's territory.

2) A standard protocol and "rules of engagement" to enable interoperability between management instrumentation and extensible agents.

3) Mechanisms for independently developed subagents to integrate into the extensible agent on a particular managed node in such a way that they need not be aware of any other existing subagents.

4) A simple, deterministic registry and dispatching algorithm. For a given extensible agent configuration, there is a single subagent who is "authoritative" for any particular region of the MIB (where "region" may extend from an entire MIB down to a single objectinstance).

5) Performance considerations. It is likely that the master agent and all subagents will reside on the same host, and in such cases Agentx is more a form of inter-process communication than a traditional communications protocol.

Some of the design decisions made with this in mind include:

- 32-bit alignment of data within PDUs

- Native byte-order encoding by subagents

- Large AgentX PDU payload sizes.

4.4. Non-Goals

1) Subagent-to-subagent communication. This is out of scope, due to the security ramifications and complexity involved.

2) Subagent access (via the master agent) to MIB variables. This is not addressed, since various other mechanisms are available and it was not a fundamental requirement.

3) The ability to accommodate every conceivable extensible agent configuration option. This was the most contentious aspect in the development of this protocol. In essence, certain features currently available in some commercial extensible agent products are not included in Agentx. Although useful or even vital in some implementation strategies, the rough consensus was that these features were not appropriate for an Internet standard, or not 
typically required for independently developed subagents to coexist. The set of supported extensible agent configurations is described above, in section 4.2, "Applicability".

Some possible future version of the Agentx protocol may provide coverage for one or more of these "non-goals" or for new goals that might be identified after greater deployment experience.

\section{Agentx Encodings}

AgentX PDUs consist of a common header, followed by PDU-specific data of variable length. Unlike SNMP PDUs, AgentX PDUs are not encoded using the BER (as specified in ISO 8824 [18]), but are transmitted as a contiguous byte stream. The data within this stream is organized to provide natural alignment with respect to the start of the PDU, permitting direct (integer) access by the processing entities.

The first four fields in the header are single-byte values. A bit (NETWORK_BYTE_ORDER) in the third field (h.flags) is used to indicate the byte ordering of all multi-byte integer values in the PDU, including those which follow in the header itself. This is described in more detail in Section 6.1, "AgentX PDU Header", below.

PDUs are depicted in this memo using the following convention (where byte 1 is the first transmitted byte):

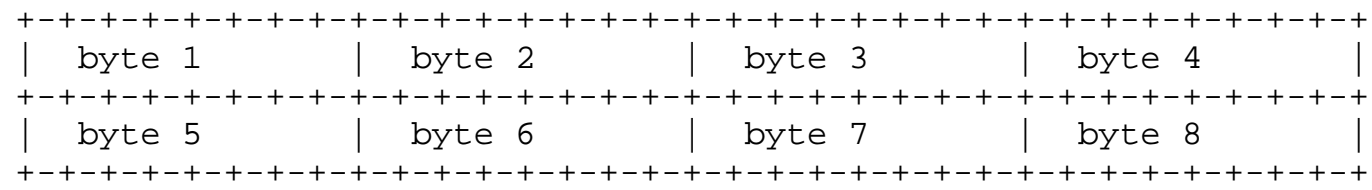

Fields marked "<reserved>" are reserved for future use and must be zero-filled.

5.1. Object Identifier

An object identifier is encoded as a 4-byte header, followed by a variable number of contiguous 4-byte fields representing subidentifiers. This representation (termed Object Identifier) is as follows: 
Object Identifier

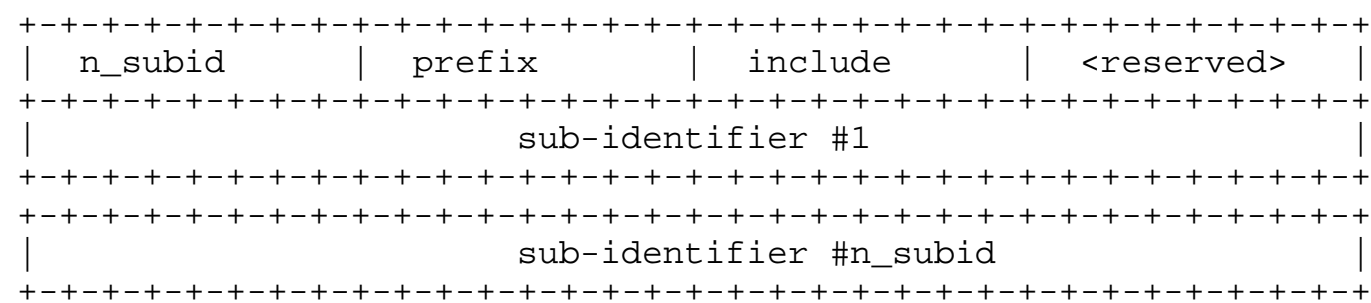

Object Identifier header fields:

n_subid

The number (0-128) of sub-identifiers in the object identifier. An ordered list of "n_subid" 4-byte sub-identifiers follows the 4-byte header.

prefix

An unsigned value used to reduce the length of object identifier encodings. A non-zero value "x" is interpreted as the first sub-identifier after "internet" (1.3.6.1), and indicates an implicit prefix "internet.x" to the actual subidentifiers encoded in the object Identifier. For example, a prefix field value 2 indicates an implicit prefix "1.3.6.1.2". A value of 0 in the prefix field indicates there is no prefix to the sub-identifiers.

include

Used only when the object Identifier is the start of a SearchRange, as described in section 5.2, "SearchRange".

sub-identifier $1,2, \ldots$ n_subid

A 4-byte unsigned integer, encoded according to the header's NETWORK_BYTE_ORDER bit.

A null Object Identifier consists of the 4-byte header with all bytes set to 0 . 


\section{Examples:}

sysDescr.0 (1.3.6.1.2.1.1.1.0)

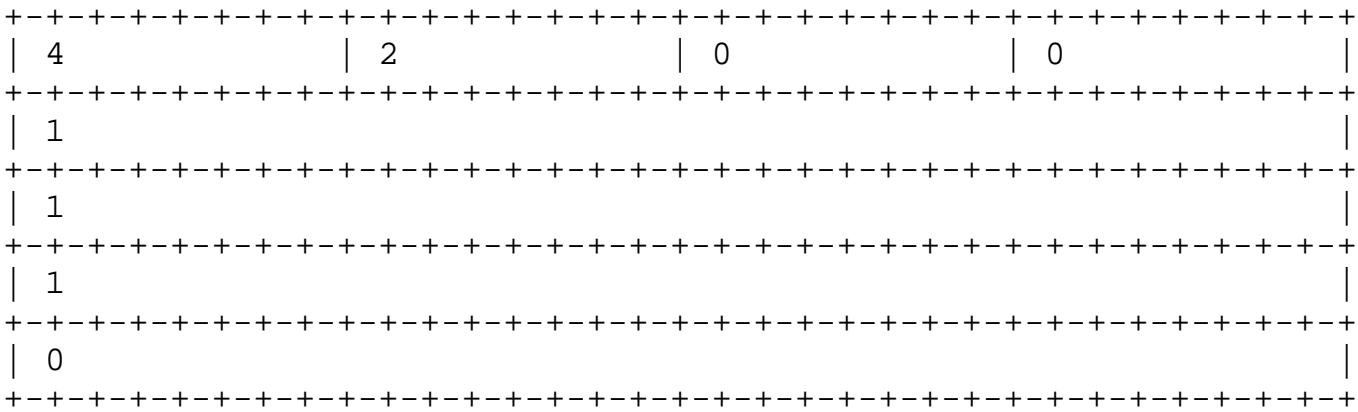

\section{2 .3 .4}

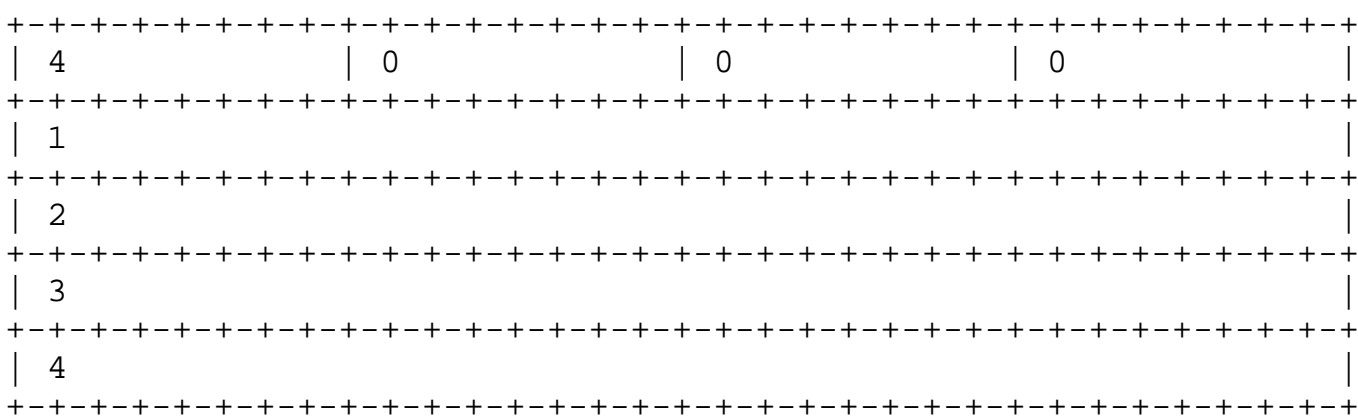

\subsection{SearchRange}

A SearchRange consists of two object Identifiers. In its communication with a subagent, the master agent uses a SearchRange to identify a requested variable binding, and, in GetNext and GetBulk operations, to set an upper bound on the names of managed object instances the subagent may send in reply.

The first Object Identifier in a SearchRange (called the starting OID) indicates the beginning of the range. It is frequently (but not necessarily) the name of a requested variable binding.

The "include" field in this OID's header is a boolean value (0 or 1$)$ indicating whether or not the starting OID is included in the range.

The second object identifier (ending OID) indicates the non-inclusive end of the range, and its "include" field is always 0 . A null value for ending OID indicates an unbounded SearchRange. 
Example: To indicate a search range from 1.3.6.1.2.1.25.2

(inclusive) to 1.3.6.1.2.1.25.2.1 (exclusive), the searchRange would be:

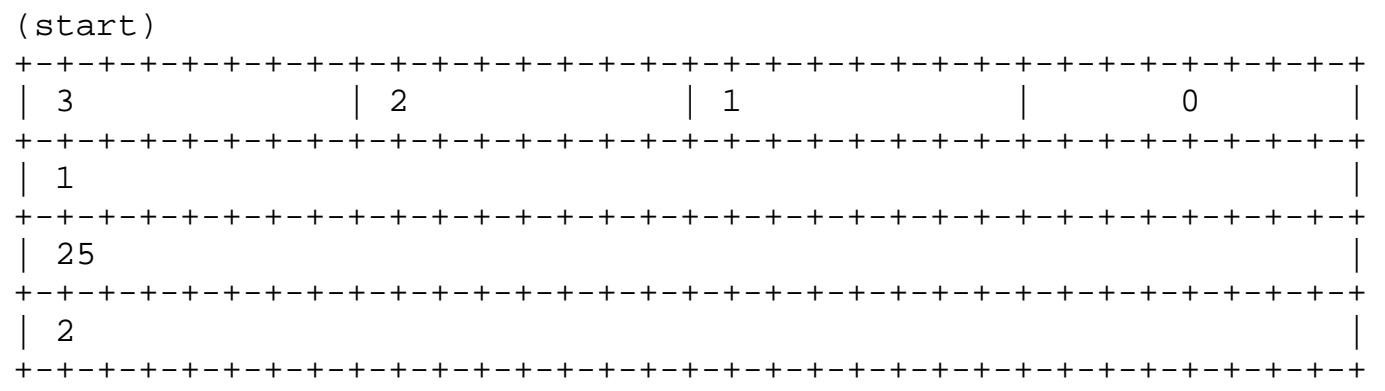

(end)

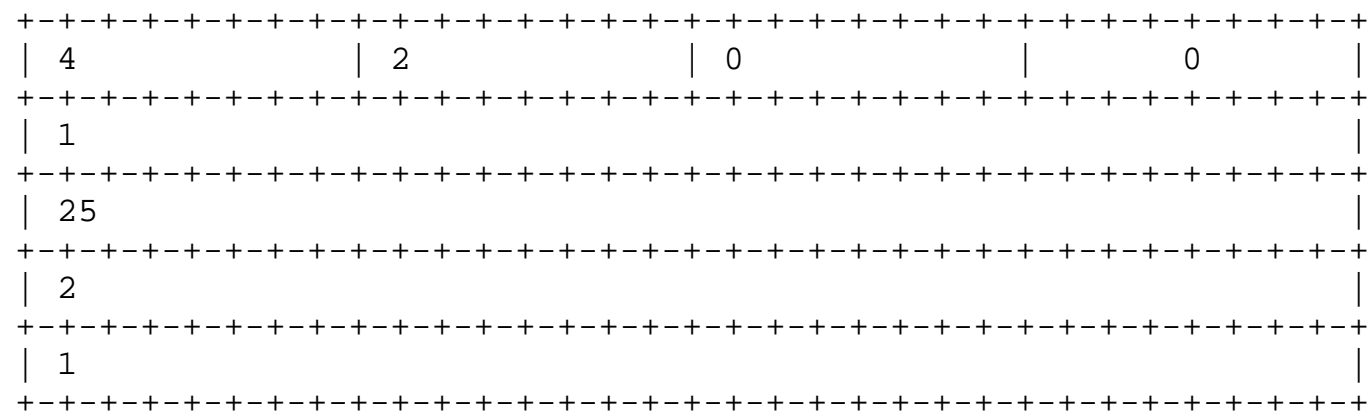

A SearchRangelist is a contiguous list of SearchRanges.

\subsection{Octet String}

An octet string is represented by a contiguous series of bytes, beginning with a 4-byte integer (encoded according to the header's NETWORK_BYTE_ORDER bit) whose value is the number of octets in the octet string, followed by the octets themselves. This representation is termed an octet String. If the last octet does not end on a 4byte offset from the start of the Octet String, padding bytes are appended to achieve alignment of following data. This padding must be added even if the octet string is the last item in the PDU. Padding bytes must be zero filled. 


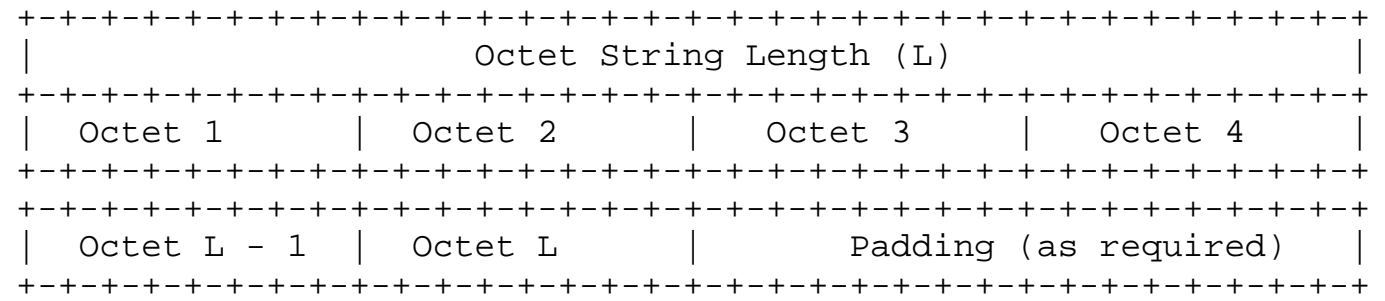

A null Octet String consists of a 4-byte length field set to 0 .

\subsection{Value Representation}

Variable bindings may be encoded within the variable-length portion of some PDUs. The representation of a variable binding (termed a VarBind) consists of a 2-byte type field, a name (Object Identifier), and the actual value data.

VarBind

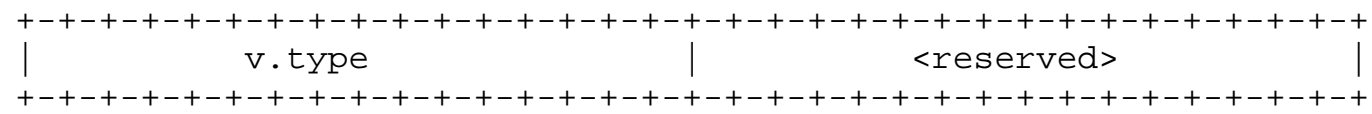

( $v$. name)

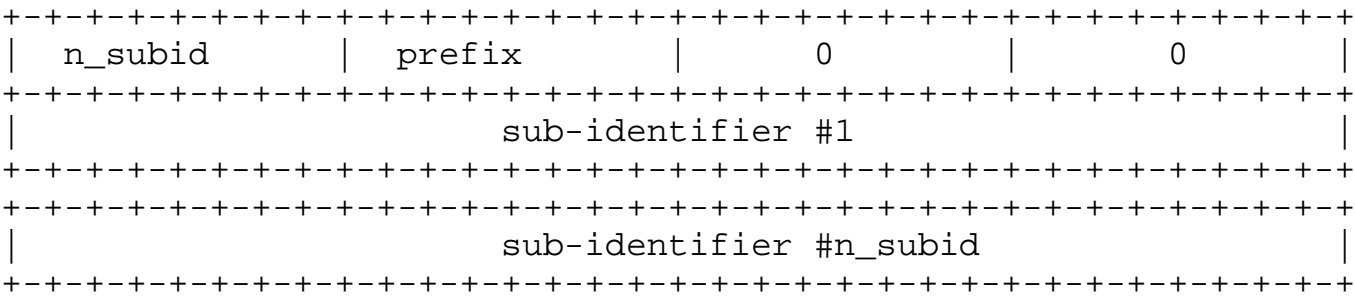

(v.data)

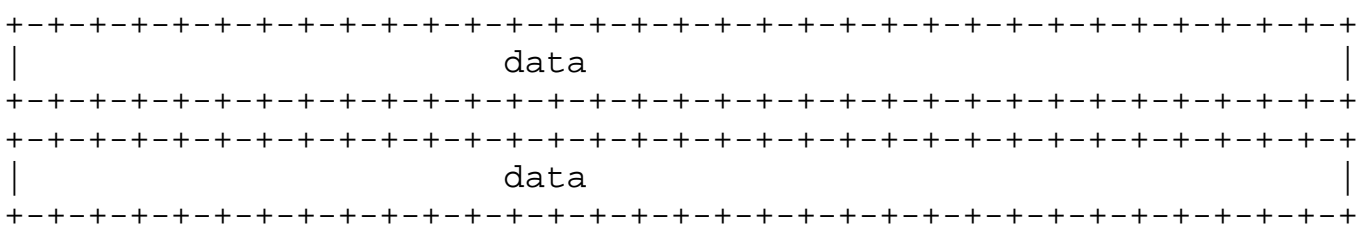

VarBind fields:

$$
\text { v. type }
$$

Indicates the variable binding's syntax, and must be one of the following values: 


$\begin{array}{lr}\text { Integer } & (2), \\ \text { Octet String } & (4), \\ \text { Null } & (5), \\ \text { Object Identifier } & (6), \\ \text { IpAddress } & (64), \\ \text { Counter32 } & (65), \\ \text { Gauge32 } & (66), \\ \text { TimeTicks } & (67), \\ \text { Opaque } & (68), \\ \text { Counter64 } & (70), \\ \text { noSuchObject } & (128), \\ \text { noSuchInstance } & (129), \\ \text { endOfMibView } & (130)\end{array}$

v. name

The Object Identifier which names the variable. v.data

The actual value, encoded as follows:

- Integer, Counter32, Gauge32, and TimeTicks are encoded as 4 contiguous bytes, according to the header's NETWORK_BYTE_ORDER bit.

- Counter64 is encoded as 8 contiguous bytes, according to the header's NETWORK_BYTE_ORDER bit.

- Object Identifiers are encoded as described in section 5.1, Object Identifier.

- IpAddress, Opaque, and Octet String are all octet strings and are encoded as described in section 5.3, "Octet String", Octet String. Note that the octets used to represent IpAddress are always ordered most significant to least significant.

Value data always follows $v$.name whenever $v . t y p e$ is one of the above types. These data bytes are present even if they will not be used (as, for example, in certain types of index allocation).

- Null, noSuchobject, noSuchInstance, and endofMibView do not contain any encoded value. Value data never follows $v$.name in these cases. 
Note that the VarBind itself does not contain the value size. That information is implied for the fixed-length types, and explicitly contained in the encodings of variable-length types object Identifier and Octet string).

A VarBindList is a contiguous list of VarBinds. Within a VarBindList, a particular VarBind is identified by an index value. The first VarBind in a VarBindList has index value 1, the second has index value 2 , and so on.

6. Protocol Definitions

\subsection{AgentX PDU Header}

The AgentX PDU header is a fixed-format, 20-octet structure:

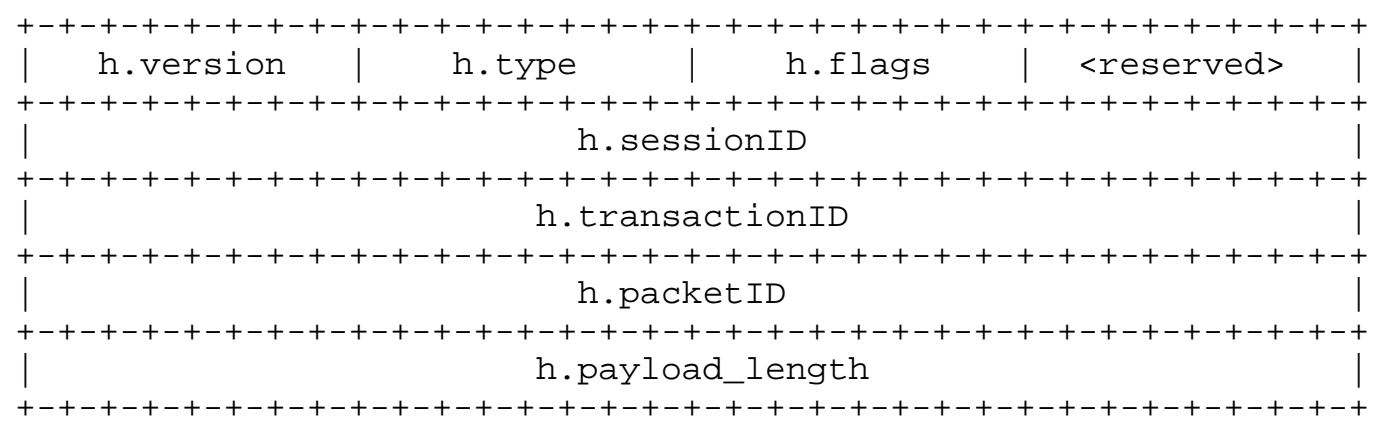

An Agentx PDU header contains the following fields:

h. version

The version of the Agentx protocol (1 for this memo).

h. type

The PDU type; one of the following values:

$\begin{array}{lc}\text { agentx-Open-PDU } & (1), \\ \text { agentx-Close-PDU } & (2), \\ \text { agentx-Register-PDU } & (3), \\ \text { agentx-Unregister-PDU } & (4), \\ \text { agentx-Get-PDU } & (5), \\ \text { agentx-GetNext-PDU } & (6), \\ \text { agentx-GetBulk-PDU } & (7), \\ \text { agentx-TestSet-PDU } & (8), \\ \text { agentx-CommitSet-PDU } & (9), \\ \text { agentx-UndoSet-PDU } & (10),\end{array}$




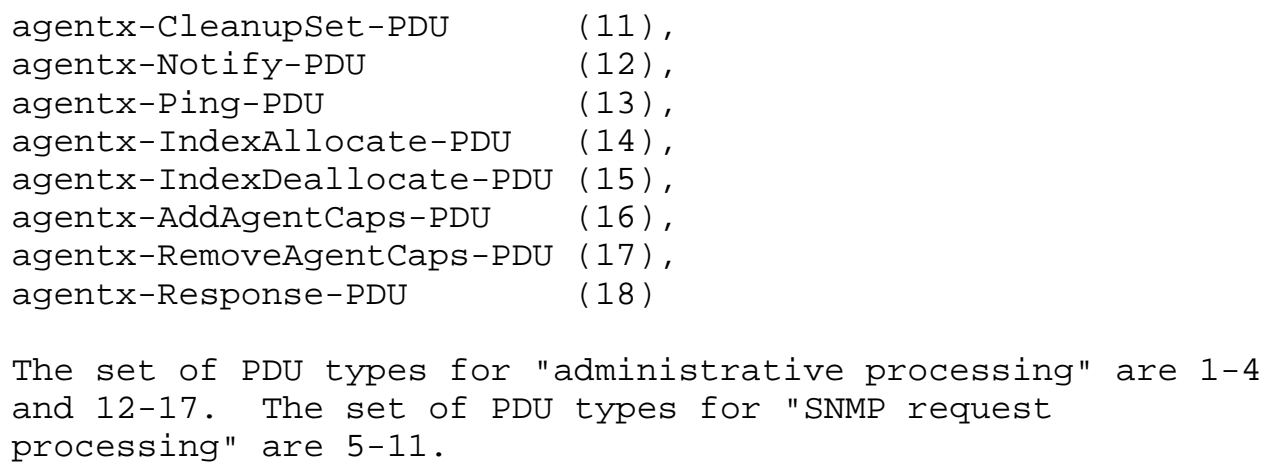

The set of PDU types for "administrative processing" are 1-4 and 12-17. The set of PDU types for "SNMP request processing" are 5-11.

h.flags

A bitmask, with bit 0 the least significant bit. The bit definitions are as follows:

$\begin{array}{ll}\text { Bit } & \text { Definition } \\ --- & -------- \\ 0 & \text { INSTANCE_REGISTRATION } \\ 1 & \text { NEW_INDEX } \\ 2 & \text { ANY_INDEX } \\ 3 & \text { NON_DEFAULT_CONTEXT } \\ 4 & \text { NETWORK_BYTE_ORDER } \\ 5-7 & \text { (reserved) }\end{array}$

The NETWORK_BYTE_ORDER bit applies to all multi-byte integer values in the entire Agentx packet, including the remaining header fields. If set, then network byte order (most significant byte first; "big endian") is used. If not set, then least significant byte first ("little endian") is used.

The NETWORK_BYTE_ORDER bit applies to all AgentX PDUs.

The NON_DEFAULT_CONTEXT bit is used only in the AgentX PDUs described in section 6.1.1, "Context".

The NEW_INDEX and ANY_INDEX bits are used only within the agentx-IndexAllocate-, and -IndexDeallocate-PDUs.

The INSTANCE_REGISTRATION bit is used only within the agentx-Register-PDU . 
h.sessionID

The session ID uniquely identifies a session over which AgentX PDUs are exchanged between a subagent and the master agent. The session ID has no significance and no defined value in the agentx-Open-PDU sent by a subagent to open a session with the master agent; in this case, the master agent will assign a unique session ID that it will pass back in the corresponding agentx-Response-PDU. From that point on, that same session ID will appear in every AgentX PDU exchanged over that session between the master and the subagent. A subagent may establish multiple Agentx sessions by sending multiple agentx-Open-PDUs to the master agent.

In master agents that support multiple transport protocols, the sessionID should be globally unique rather than unique just to a particular transport.

h.transactionID

The transaction ID uniquely identifies, for a given session, the single SNMP management request (and single SNMP PDU) with which an Agentx PDU is associated. If a single SNMP management request results in multiple AgentX PDUs being sent by the master agent with the same session ID, each of these AgentX PDUs must contain the same transaction ID; conversely, Agentx PDUs sent during a particular session, that result from distinct SNMP management requests, must have distinct transaction IDs within the limits of the 32bit field).

Note that the transaction ID is not the same as the SNMP PDU's request-id (as described in section 4.1 of RFC 1905 [13], nor is it the same as the SNMP Message's msgID (as described in section 6.2 of $\mathrm{RFC} 2572$ [11]), nor can it be, since a master agent might receive SNMP requests with the same request-ids or msgIDs from different managers.

The transaction ID has no significance and no defined value in AgentX administrative PDUs, i.e., AgentX PDUs that are not associated with an SNMP management request.

h.packetID

A packet ID generated by the sender for all AgentX PDUs except the agentx-Response-PDU. In an agentx-Response-PDU, the packet ID must be the same as that in the received AgentX PDU to which it is a response. A master agent might 
use this field to associate subagent response PDUs with their corresponding request PDUs. A subagent might use this field to correlate responses to multiple (batched) registrations.

h.payload_length

The size in octets of the PDU contents, excluding the 20byte header. As a result of the encoding schemes and PDU layouts, this value will always be either 0 , or a multiple of 4 .

\subsubsection{Context}

In the SNMPV1 or SNMPV2c, the community string may be used as an index into a local repository of configuration information that may include community profiles or more complex context information. In SNMPv3 this notion of "context" is formalized (see section 3.3 .1 in RFC 2571 [1].

Agentx provides a mechanism for transmitting a context specification within relevant PDUs, but does not place any constraints on the content of that specification.

An optional context field may be present in the agentx-Register-, UnRegister-, AddAgentCaps-, RemoveAgentCaps-, Get-, GetNext-, GetBulk-, IndexAllocate-, IndexDeallocate-, Notify-, TestSet-, and Ping- PDUs.

If the NON_DEFAULT_CONTEXT bit in the AgentX header field h.flags is clear, then there is no context field in the PDU, and the operation refers to the default context. (This does not mean there is a zerolength Octet String, it means there is no Octet string present.) If the NON_DEFAULT_CONTEXT bit is set, then a context field immediately follows the Agentx header, and the operation refers to that specific context. The context is represented as an Octet string. There are no constraints on its length or contents.

Thus, all of these AgentX PDUs (that is, those listed immediately above) refer to, or "indicate" a context, which is either the default context, or a non-default context explicitly named in the PDU.

\subsection{AgentX PDUs}

6.2.1. The agentx-Open-PDU

An agentx-Open-PDU is generated by a subagent to request establishment of an Agentx session with the master agent. 
(AgentX header)

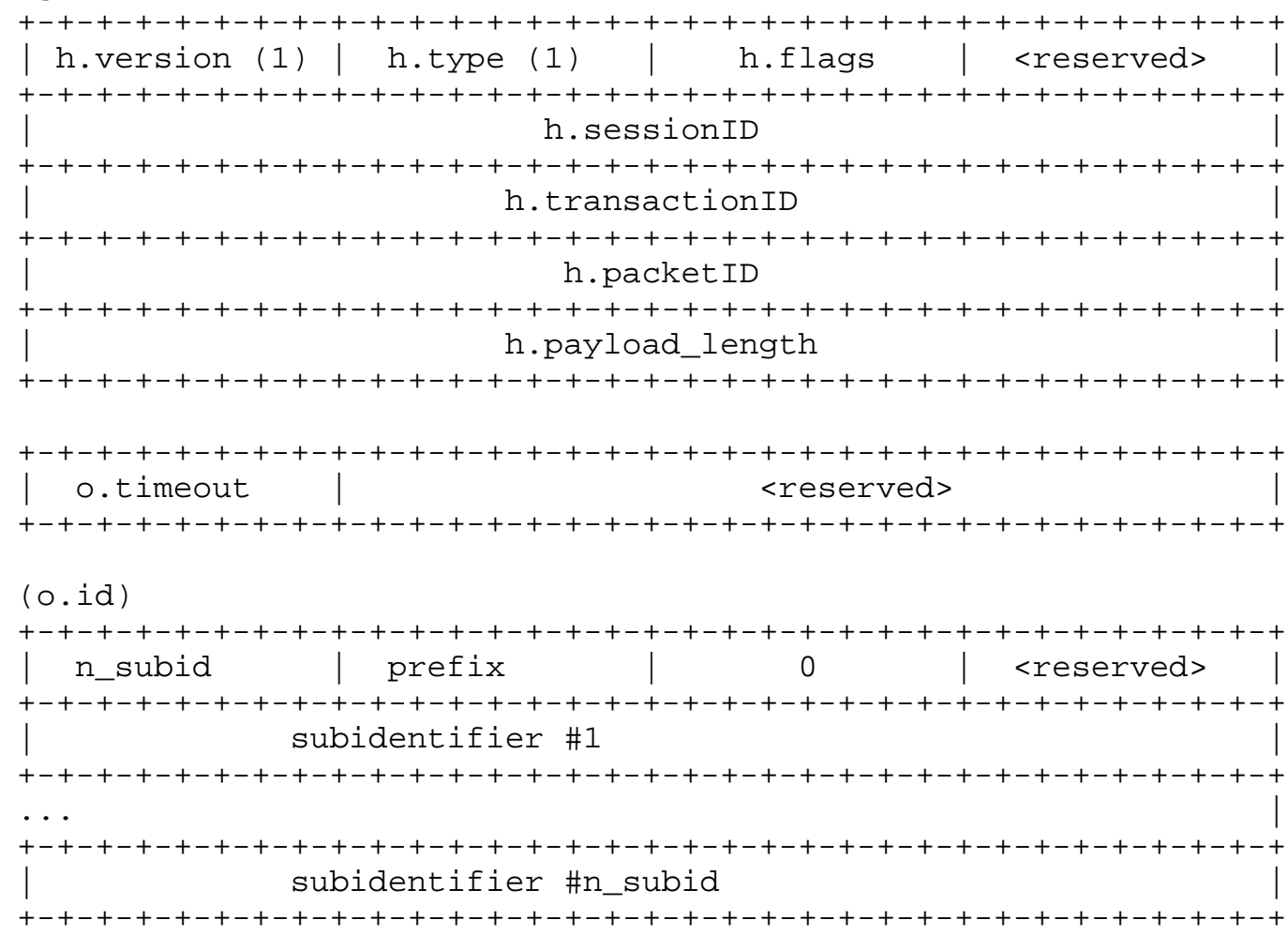

(o.descr)

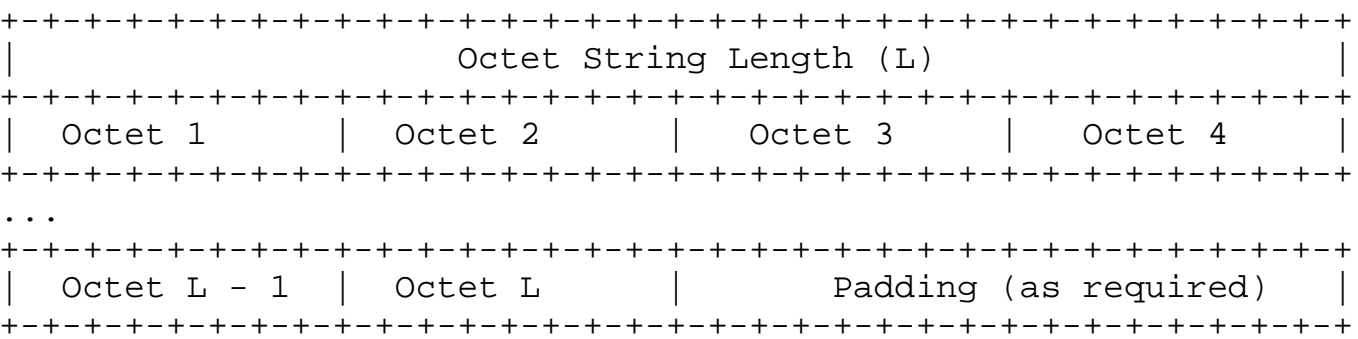

An agentx-Open-PDU contains the following fields:

o.timeout

The length of time, in seconds, that a master agent should allow to elapse after dispatching a message on a session before it regards the subagent as not responding. This is the default value for the session, and may be overridden by 
values associated with specific registered MIB regions. The default value of 0 indicates that there is no session-wide default value.

o.id

An Object Identifier that identifies the subagent.

Subagents that do not support such an notion may send a null object Identifier.

o.descr

An Octet String containing a Displaystring describing the subagent.

\subsubsection{The agentx-Close-PDU}

An agentx-Close-PDU issued by either a subagent or the master agent terminates an AgentX session.

(AgentX header)

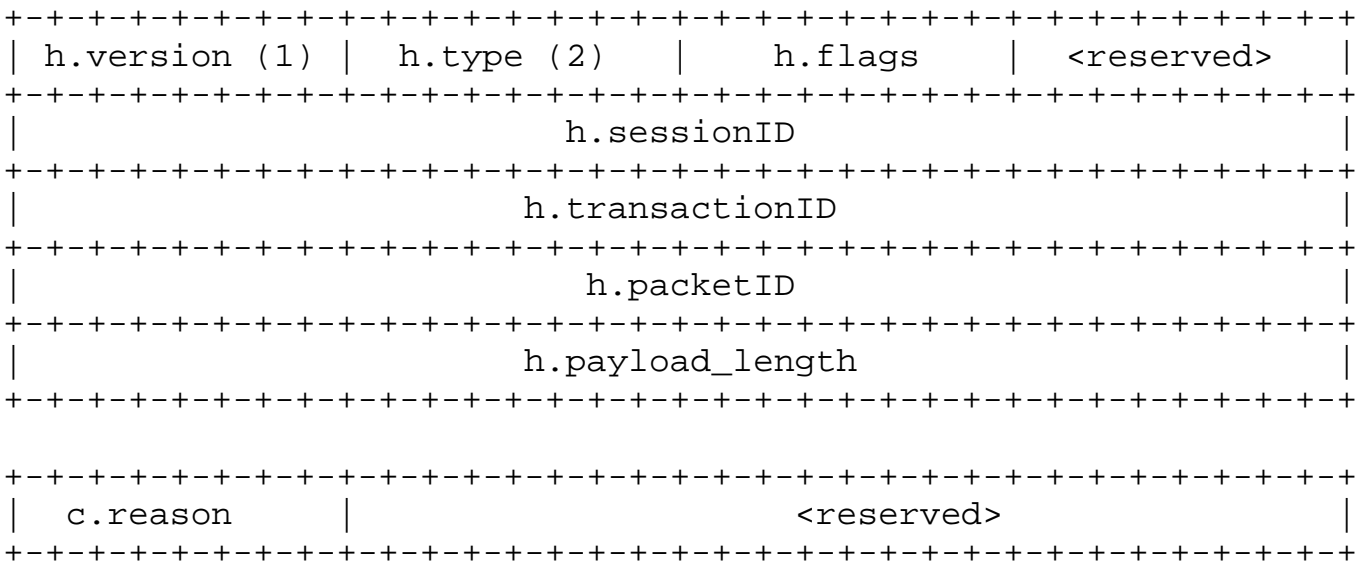

An agentx-Close-PDU contains the following field:

c.reason

An enumerated value that gives the reason that the master agent or subagent closed the Agentx session. This field may take one of the following values: 


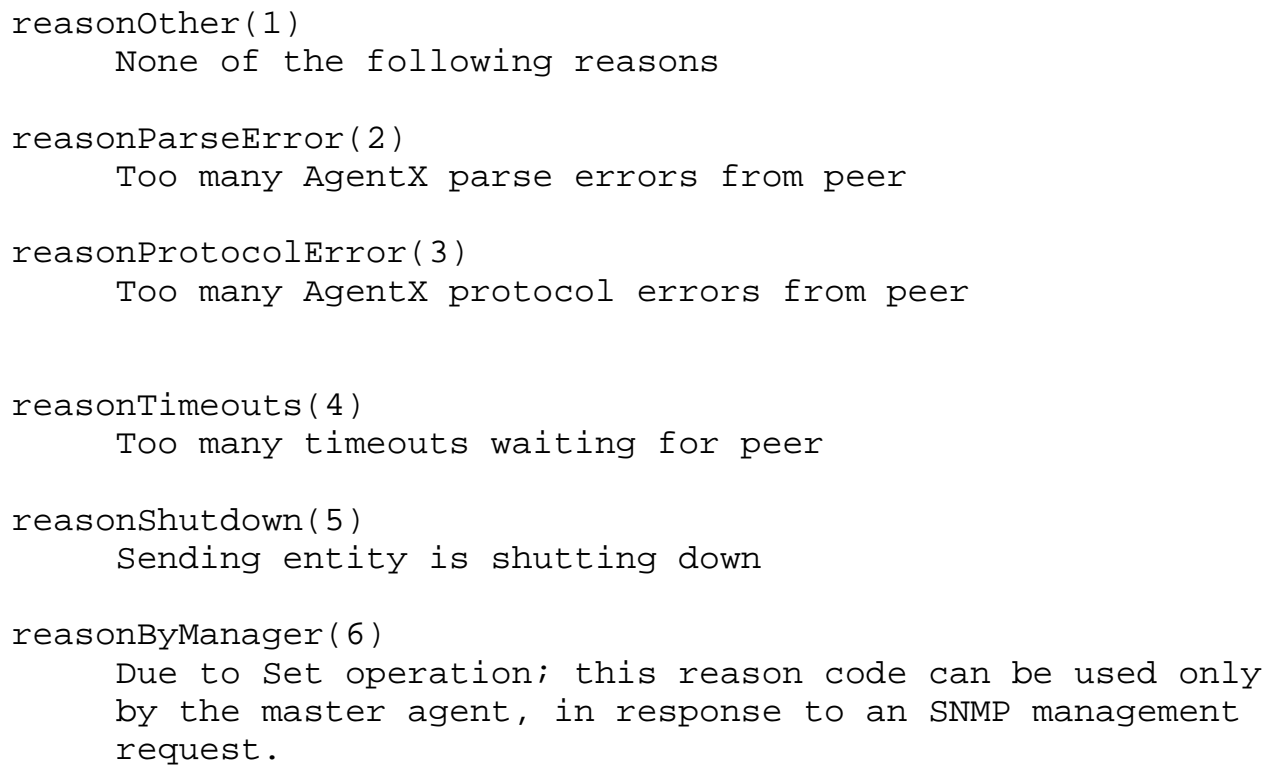

6.2.3. The agentx-Register-PDU

An agentx-Register-PDU is generated by a subagent for each region of the MIB variable naming tree (within one or more contexts) that it wishes to support.

(AgentX header)

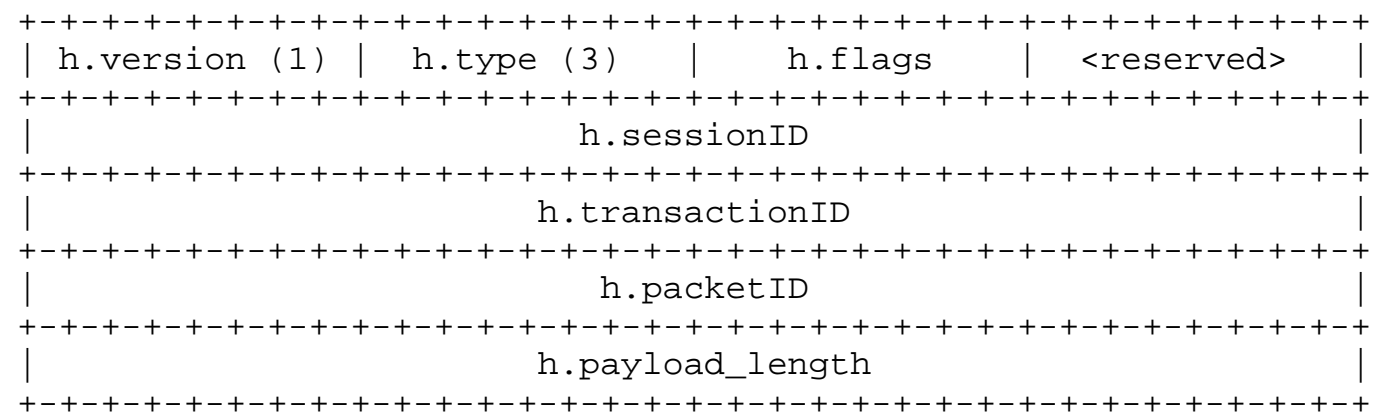

Daniele, et al. 
(r.context) (OPTIONAL)

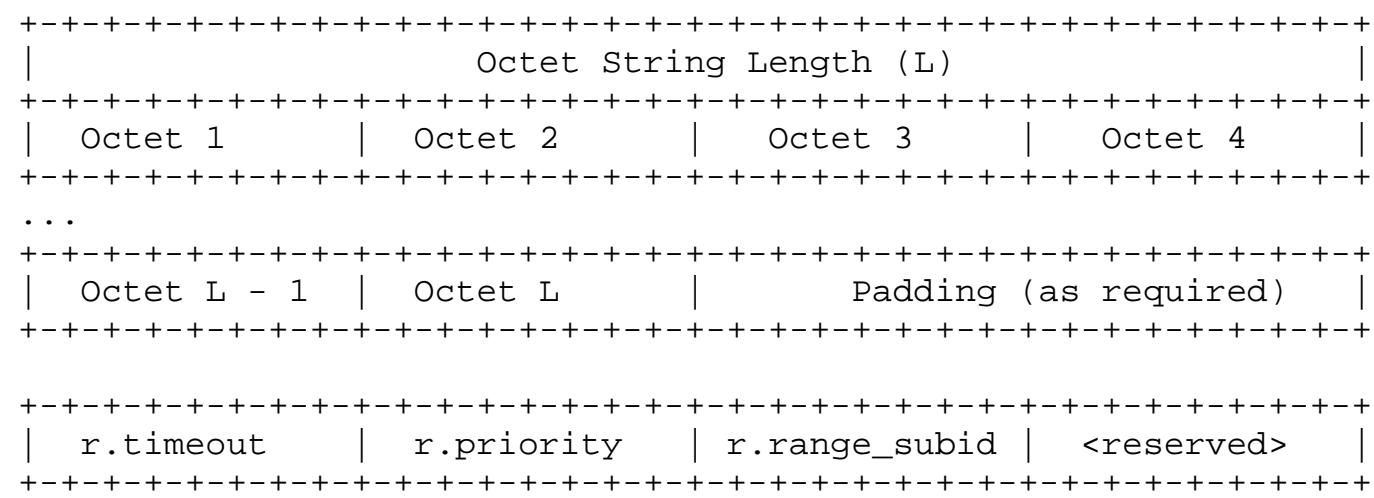

(r.subtree)

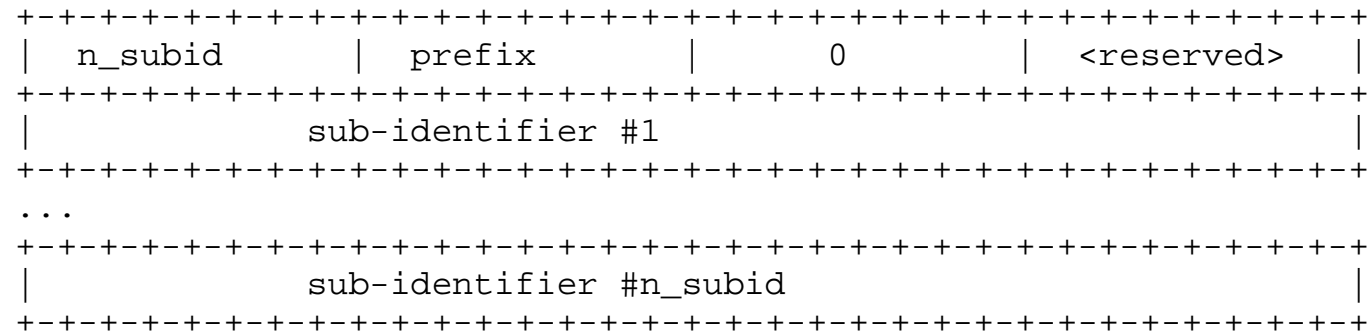

(r.upper_bound)

+-+-+-+-+-+-+-+-+-+-+-+-+-+-+-+-+-+-+-+-+-+-+-+-+-+-+-+-+-+-+-+-+
optional upper-bound sub-identifier
+-+-+-+-+-+-+-+-+-+-+-+-+-+-+-+-+-+-+-+-+-+-+-+-+-+-+-+-+-+-+-+-+

An agentx-Register-PDU contains the following fields:

r.context

An optional non-default context.

r.timeout

The length of time, in seconds, that a master agent should allow to elapse after dispatching a message on a session before it regards the subagent as not responding. r.timeout applies only to messages that concern MIB objects within r.subtree. It overrides both the session's default value (if any) indicated when the Agentx session with the master agent was established, and the master agent's default timeout. The default value for $r$.timeout is 0 (no override). 
r.priority

A value between 1 and 255, used to achieve a desired configuration when different sessions register identical or overlapping regions. Subagents with no particular knowledge of priority should register with the default value of 127 .

In the master agent's dispatching algorithm, smaller values of r.priority take precedence over larger values, as described in section 7.1.4.1, "Handling Duplicate and Overlapping subtrees".

r. subtree

An Object Identifier that names the basic subtree of a MIB region for which a subagent indicates its support. The term "subtree" is used generically here, it may represent a fully-qualified instance name, a partial instance name, a MIB table, an entire MIB, etc.

The choice of what to register is implementation-specific; this memo does not specify permissible values. Standard practice however is for a subagent to register at the highest level of the naming tree that makes sense.

Registration of fully- qualified instances is typically done only when a subagent can perform management operations only on particular rows of a conceptual table.

If $r$.subtree is in fact a fully qualified instance name, the INSTANCE_REGISTRATION bit in h.flags must be set, otherwise it must be cleared. The master agent may save this information to optimize subsequent operational dispatching.

r.range_subid

Permits specifying a range in place of one of $r$.subtree's sub-identifiers. If this value is 0 , no range is being specified and there is no r.upper_bound field present in the PDU. In this case the MIB region being registered is the single subtree named by $r$.subtree.

Otherwise the "r.range_subid"-th sub-identifier in r.subtree is a range lower bound, and the range upper bound subidentifier ( $r$.upper_bound) immediately follows $r$.subtree. In this case the MIB region being registered is the union of the subtrees formed by enumerating this range. 
Note that r.range_subid indicates the (1-based) index of this sub-identifier within the OID represented by r.subtree, regardless of whether or not $r$.subtree is encoded using a prefix. (See the example below.)

r.upper_bound

The upper bound of a sub-identifier's range. This field is present only if r.range_subid is not 0 .

The use of r.range_subid and r.upper_bound provide a general shorthand mechanism for specifying a MIB region. For example, if r.subtree is the OID 1.3.6.1.2.1.2.2.1.1.7, r.range_subid is 10 , and $r$.upper_bound is 22 , the specified MIB region can be denoted 1.3.6.1.2.1.2.2.1.[1-22].7. Registering this region is equivalent to registering the union of subtrees

$1.3 \cdot 6 \cdot 1 \cdot 2 \cdot 1 \cdot 2 \cdot 2 \cdot 1 \cdot 1 \cdot 7$

$1.3 \cdot 6 \cdot 1 \cdot 2 \cdot 1 \cdot 2 \cdot 2 \cdot 1 \cdot 2 \cdot 7$

$1.3 \cdot 6 \cdot 1 \cdot 2 \cdot 1 \cdot 2 \cdot 2 \cdot 1 \cdot 3 \cdot 7$

...

$1 \cdot 3 \cdot 6 \cdot 1 \cdot 2 \cdot 1 \cdot 2 \cdot 2 \cdot 1 \cdot 22 \cdot 7$

One expected use of this mechanism is registering a conceptual row with a single PDU. In the example above, the MIB region happens to be row 7 of the RFC 1573 iftable. The actual PDU would be:

(Agentx header)

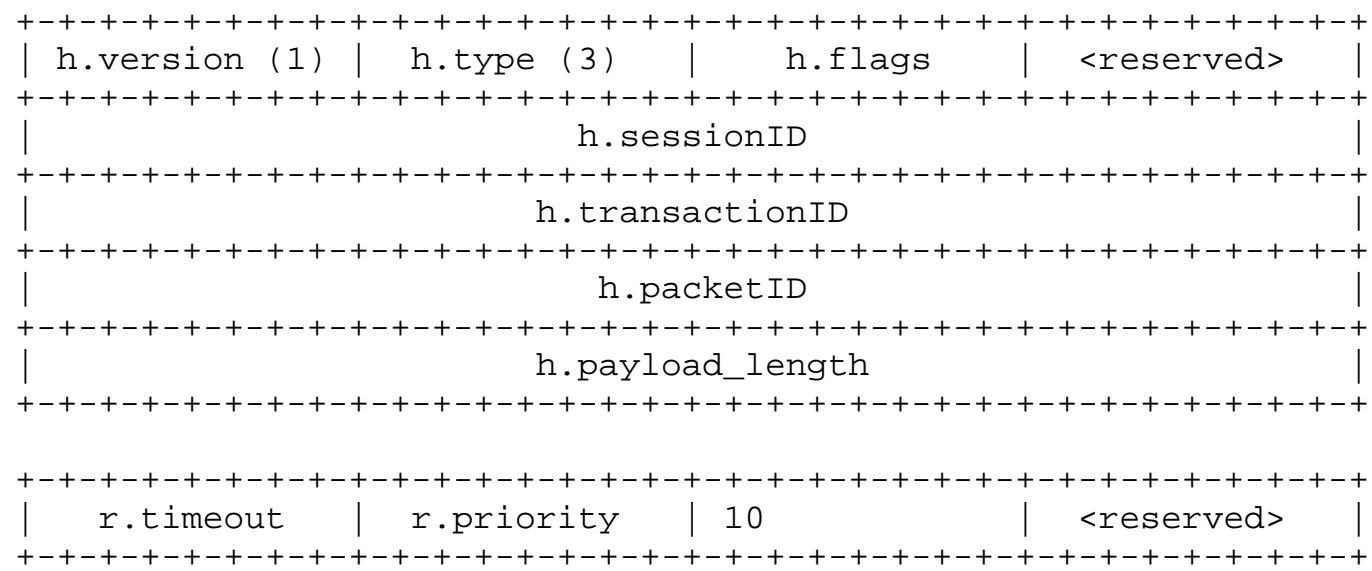


(r.subtree)

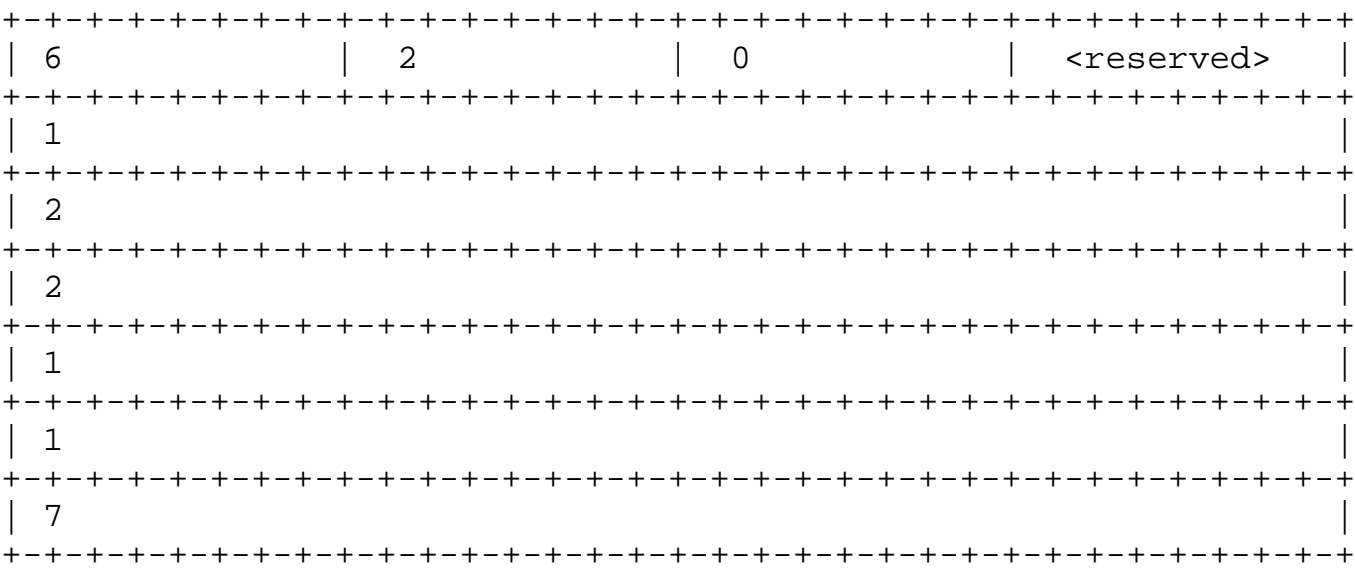

(r.upper_bound)

$+-+-+-+-+-+-+-+-+-+-+-+-+-+-+-+-+-+-+-+-+-+-+-+-+-+-+-+-+-+-+-+-+$ 22

$+-+-+-+-+-+-+-+-+-+-+-+-+-+-+-+-+-+-+-+-+-+-+-+-+-+-+-+-+-+-+-+-+$

Note again that here r.range_subid is 10 , even though n_subid in r.subtree is only 6.

r.range_subid may be used at any level within a subtree, it need not represent row-level registration. This mechanism may be used in any way that is convenient for a subagent to achieve its registrations.

\subsubsection{The agentx-Unregister-PDU}

The agentx-Unregister-PDU is sent by a subagent to remove a MIB region that was previously registered on this session.

(AgentX header)

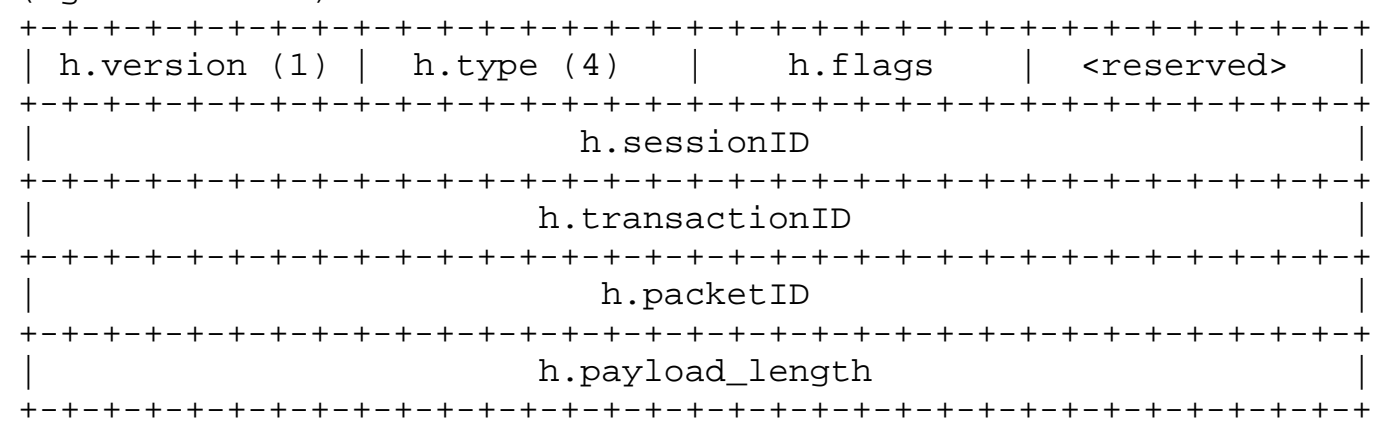

Daniele, et al. 


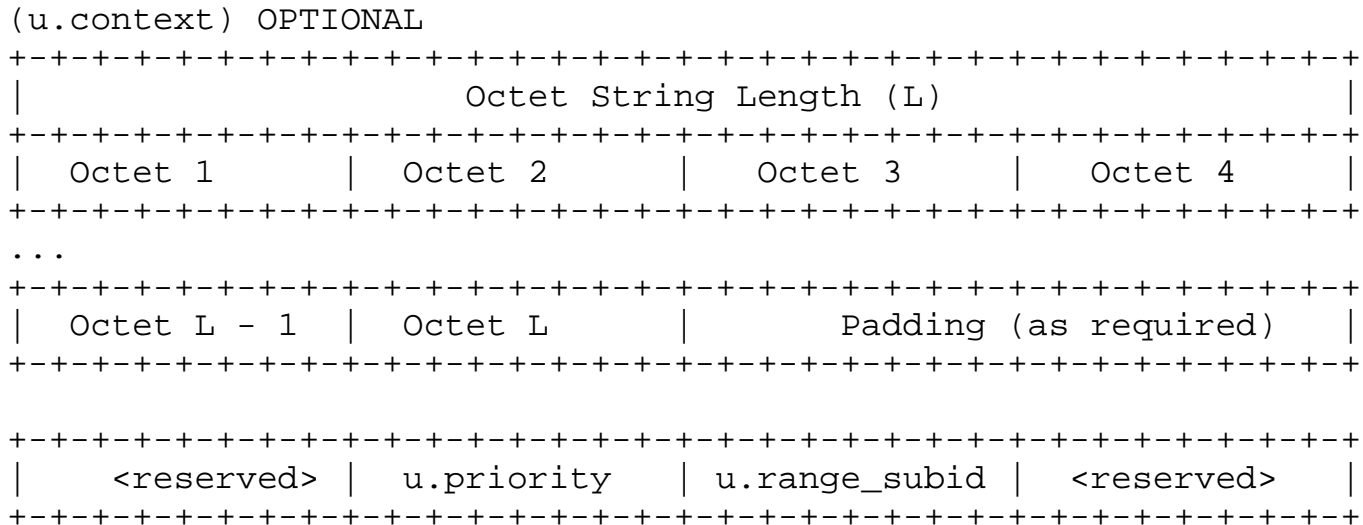

An agentx-Unregister-PDU contains the following fields:

u. context

An optional non-default context.

u.priority

The priority at which this region was originally registered. u. subtree

Indicates a previously-registered region of the MIB that a subagent no longer wishes to support. 
u.range_subid

Indicates a sub-identifier in u.subtree is a range lower bound.

u. upper_bound

The upper bound of the range sub-identifier. This field is present in the PDU only if u.range_subid is not 0 .

6.2.5. The agentx-Get-PDU

(AgentX header)

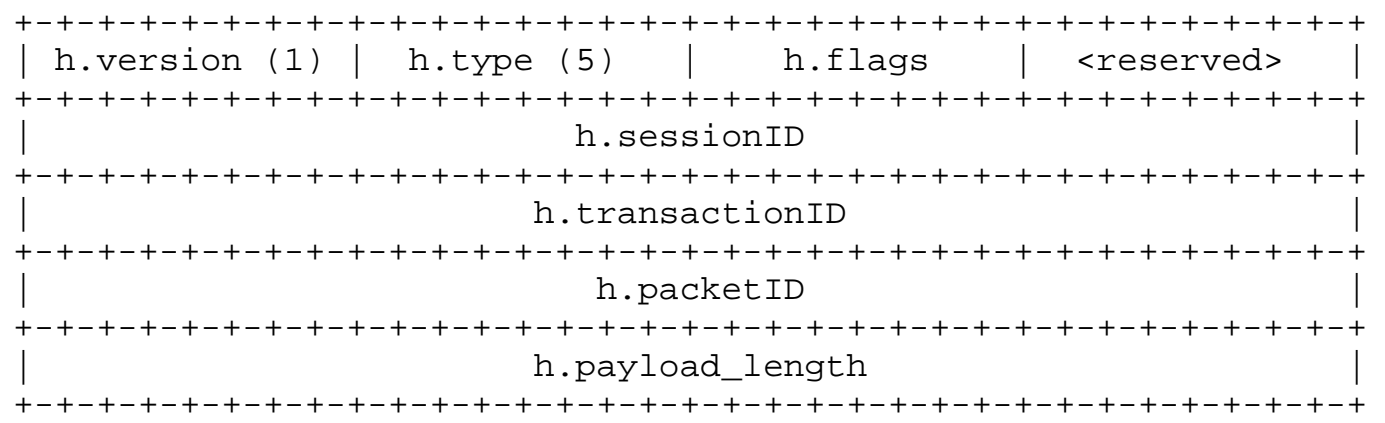

(g.context) OPTIONAL

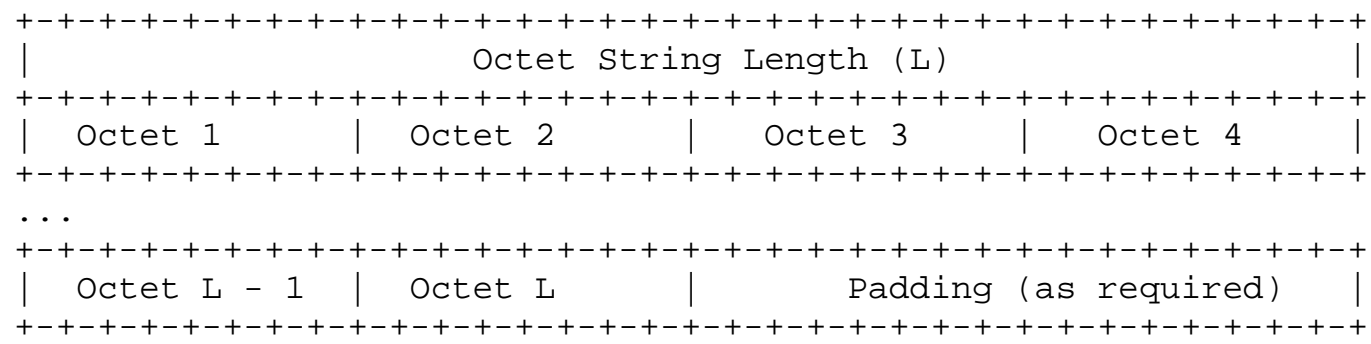

$(g \cdot s r)$

(start 1)

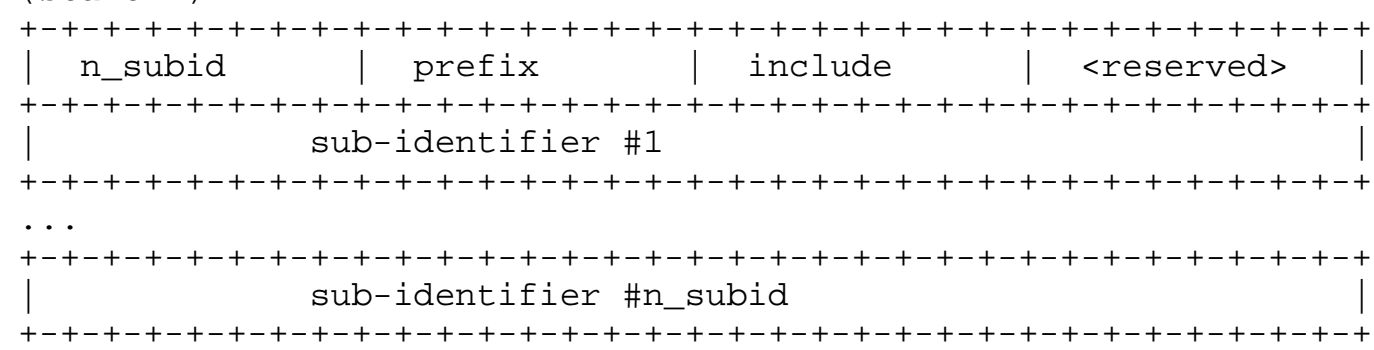

Daniele, et al. 
(end 1)

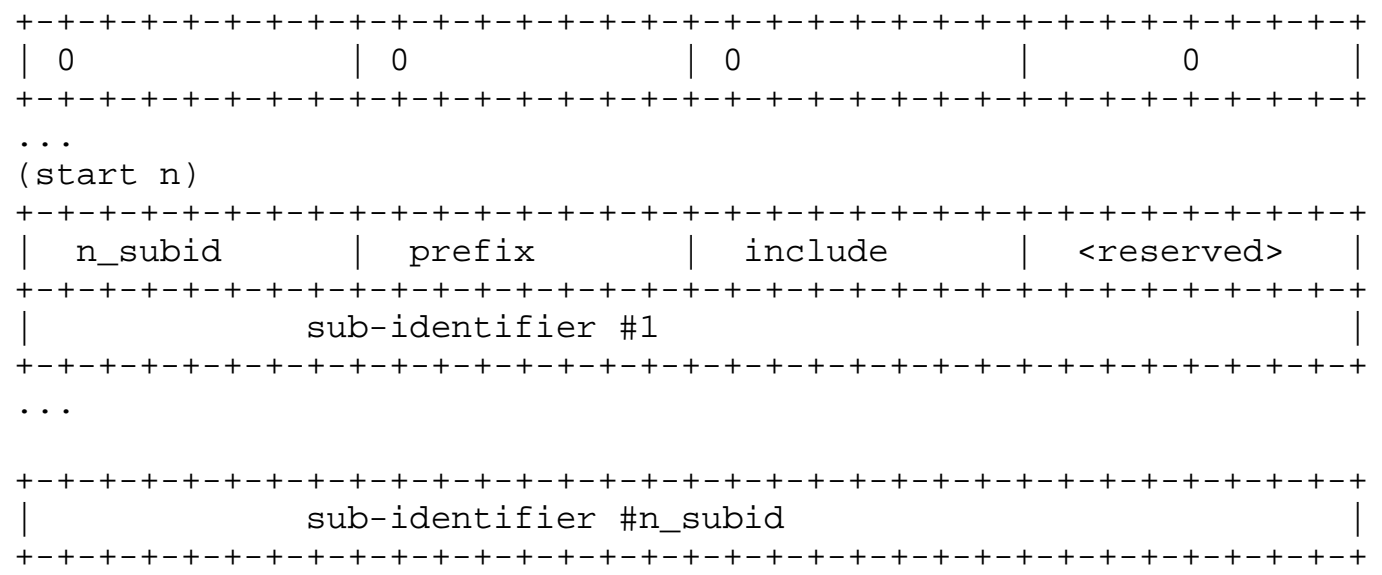

(end $\mathrm{n}$ )

\begin{tabular}{|c|c|c|c|}
\hline 0 & 0 & 0 & 0 \\
\hline
\end{tabular}

An agentx-Get-PDU contains the following fields:

g. context

An optional non-default context.

g.sr

A SearchRangelist containing the requested variables for this session. Within the agentx-Get-PDU, the Ending OIDs within SearchRanges are null-valued object Identifiers.

\subsubsection{The agentx-GetNext-PDU}

(AgentX header)

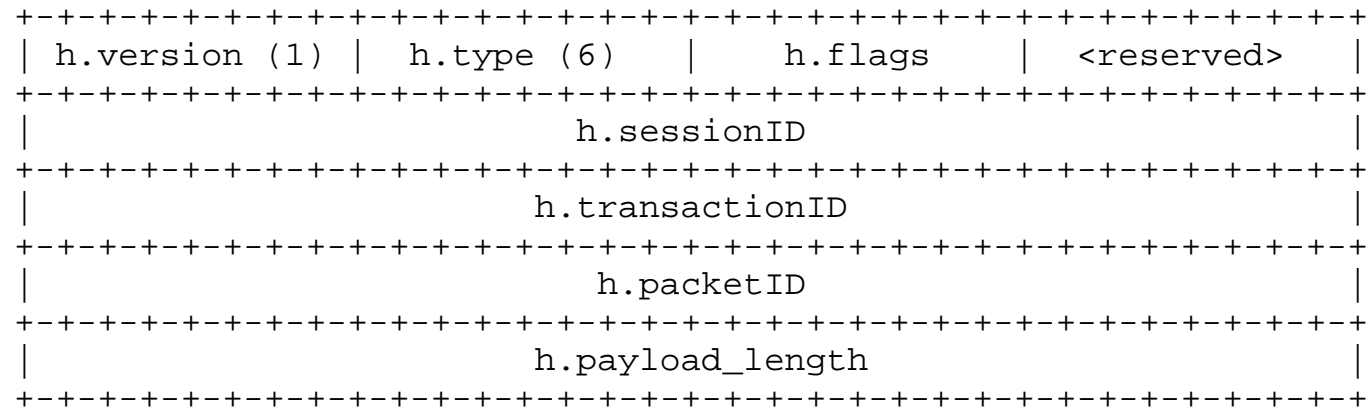


(g. context) OPTIONAL

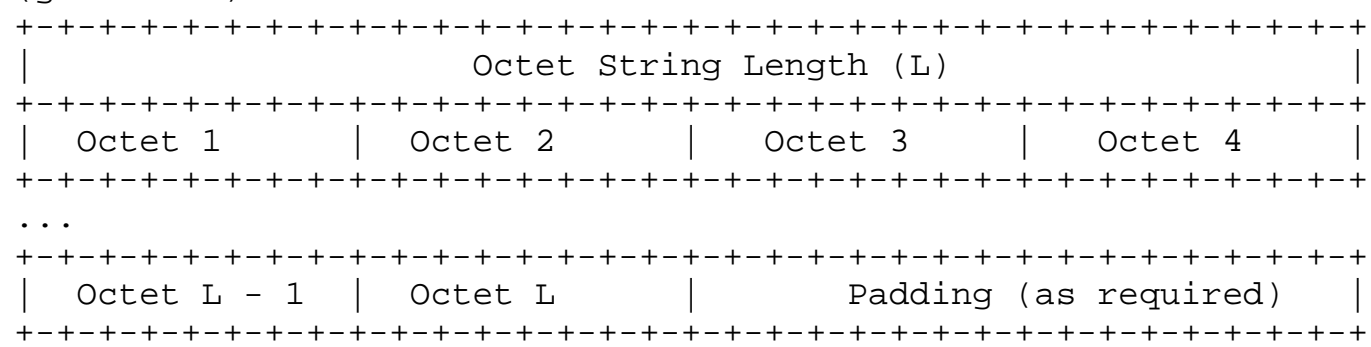

$(g \cdot s r)$

(start 1)

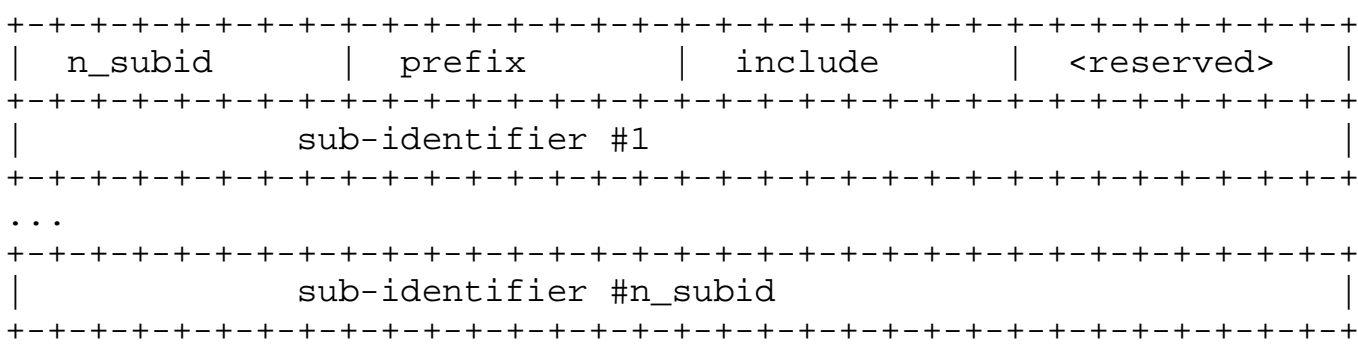

(end 1)

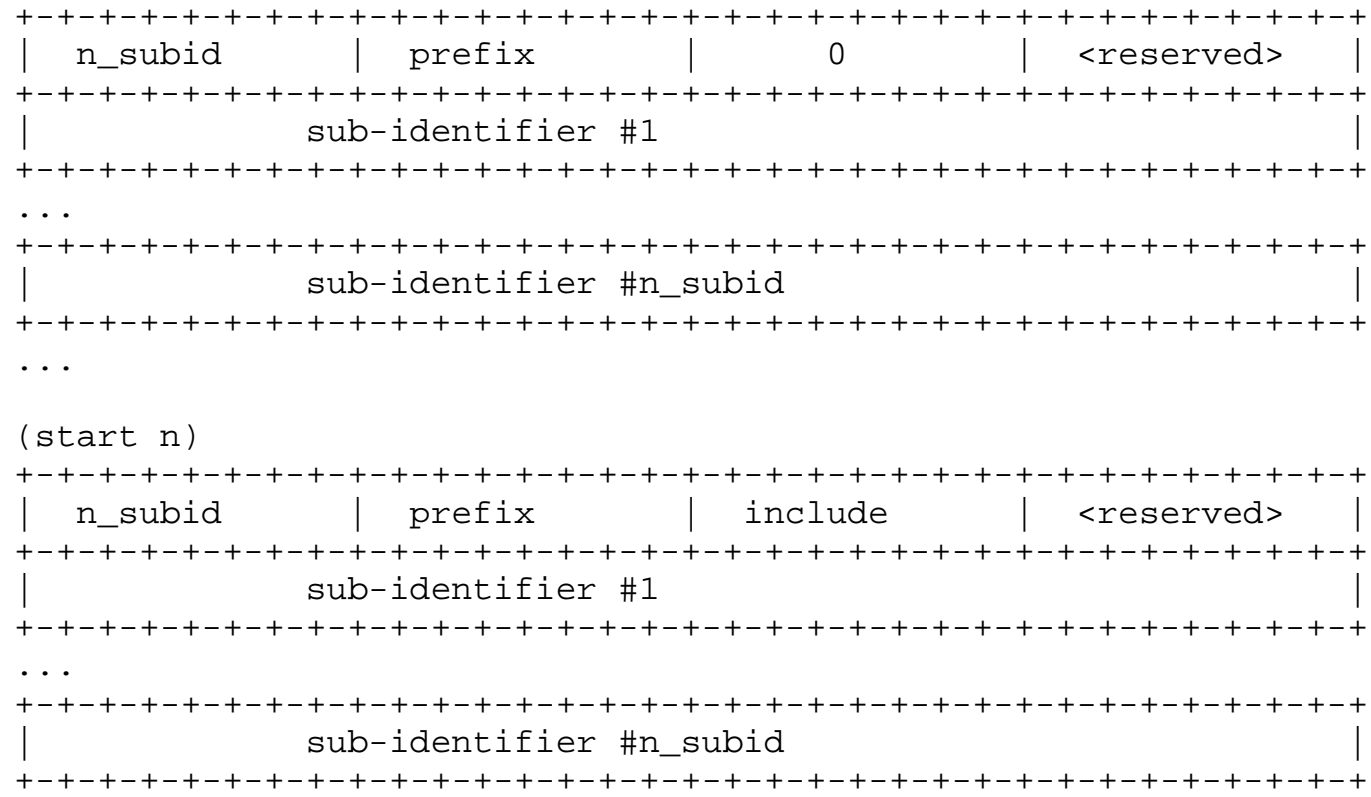


(end $\mathrm{n}$ )

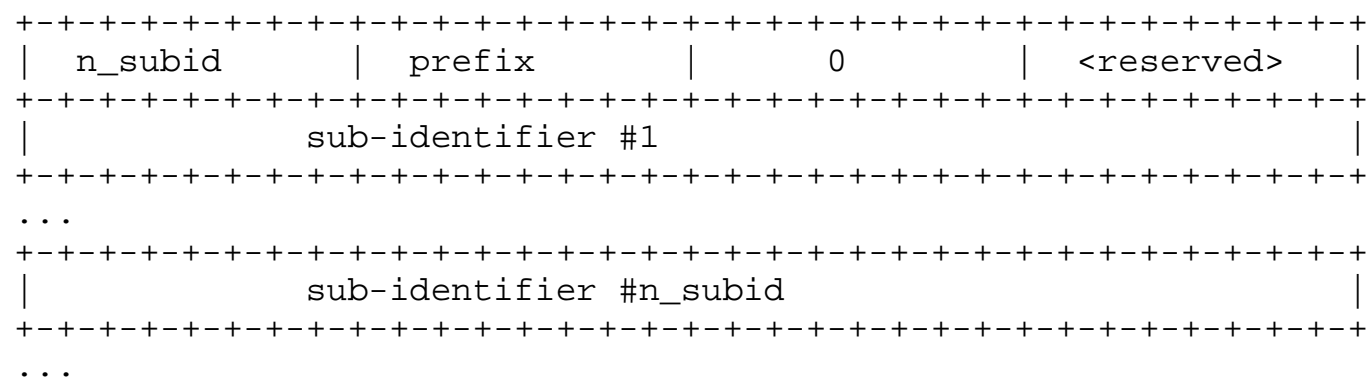

$\bullet \cdot$

An agentx-GetNext-PDU contains the following fields:

g. context

An optional non-default context.

$g \cdot s r$

A SearchRangelist containing the requested variables for this session.

6.2.7. The agentx-GetBulk-PDU

(AgentX header)

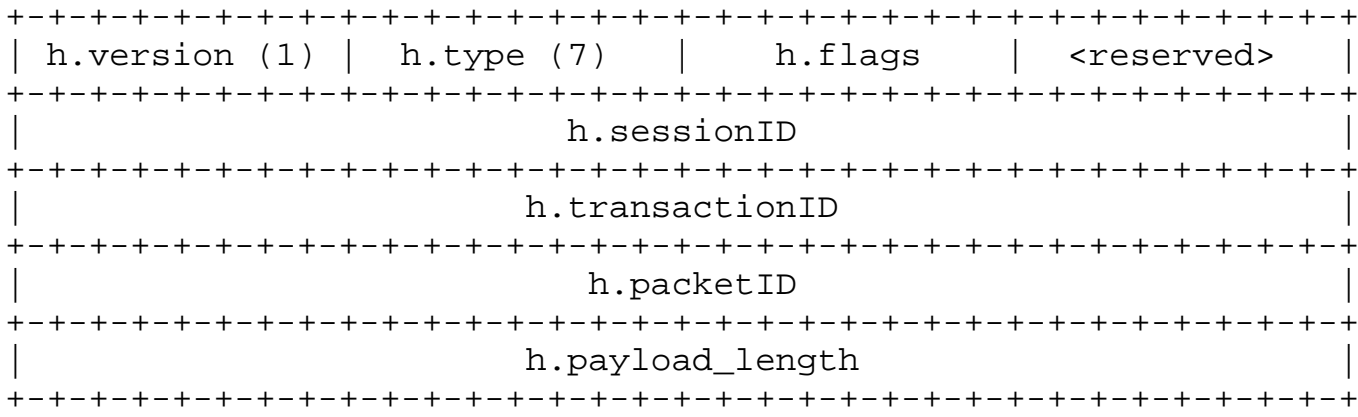

Daniele, et al.

Standards Track

[Page 32] 
(g. context) OPTIONAL

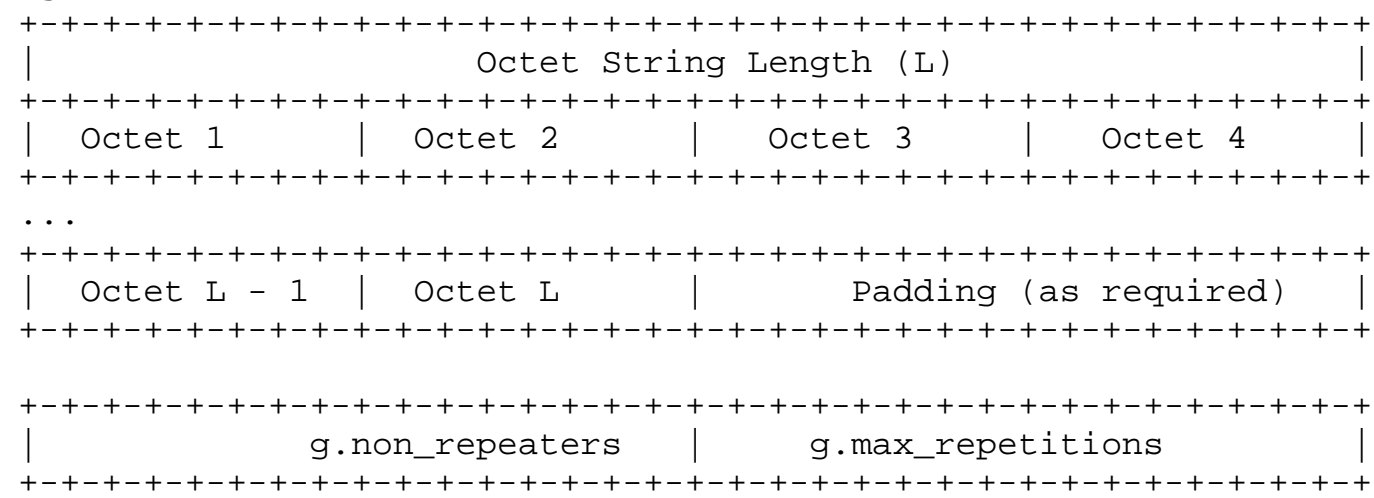

$(g \cdot s r)$

...

An agentx-GetBulk-PDU contains the following fields:

g. context

An optional non-default context.

g.non_repeaters

The number of variables in the SearchRangelist that are not repeaters.

g.max_repetitions

The maximum number of repetitions requested for repeating variables.

$g \cdot s r$

A SearchRangelist containing the requested variables for this session. 


\subsubsection{The agentx-TestSet-PDU}

(AgentX header)

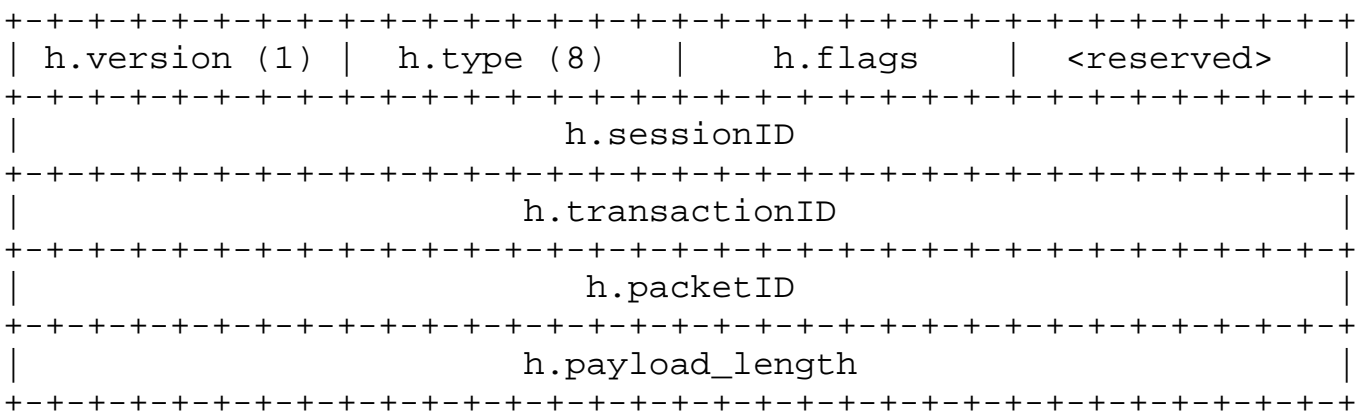

(t. context) OPTIONAL

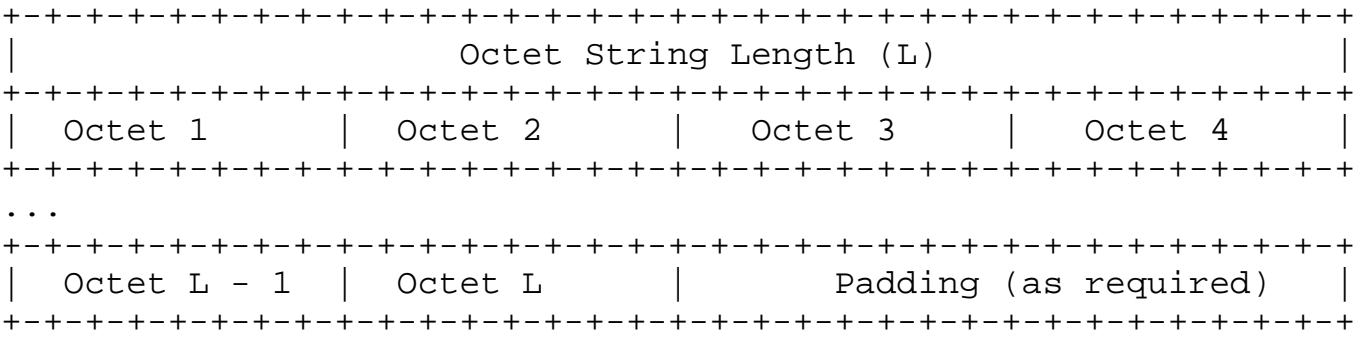

$(t \cdot v b)$

(VarBind 1)

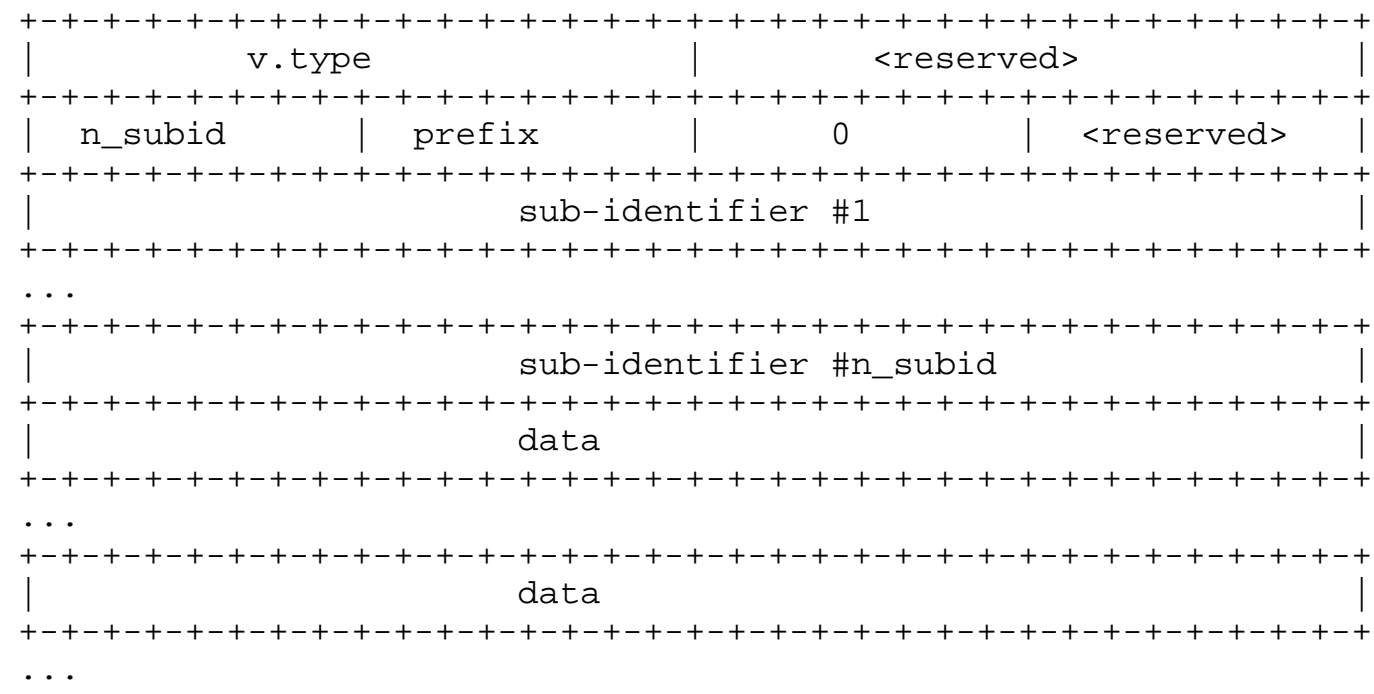

Daniele, et al. 


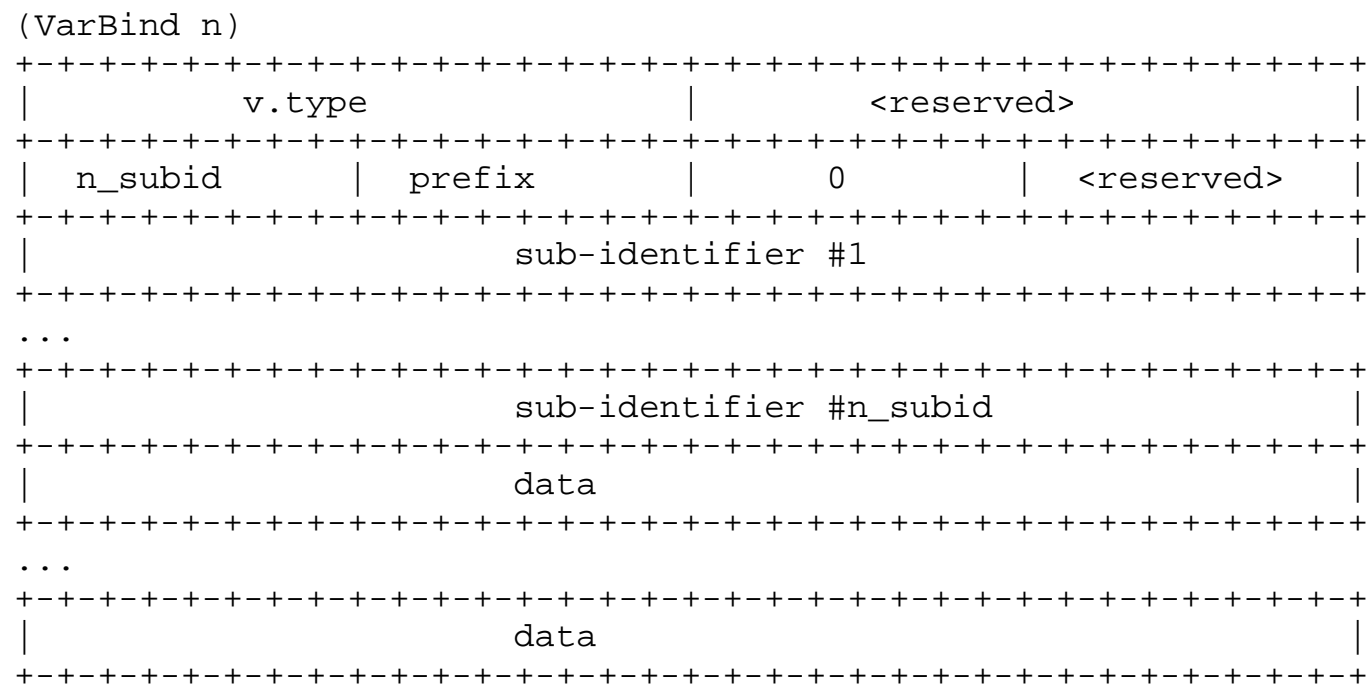

An agentx-Testset-PDU contains the following fields:

t.context

An optional non-default context.

$t \cdot v b$

A VarBindList containing the requested VarBinds for this subagent.

6.2.9. The agentx-CommitSet, -UndoSet, -CleanupSet PDUs

These PDUs consist of the Agentx header only.

The agentx-CommitSet-, -UndoSet-, and-Cleanup-PDUs are used in processing an SNMP SetRequest operation. 


\subsubsection{The agentx-Notify-PDU}

An agentx-Notify-PDU is sent by a subagent to cause the master agent to forward a notification.

(AgentX header)

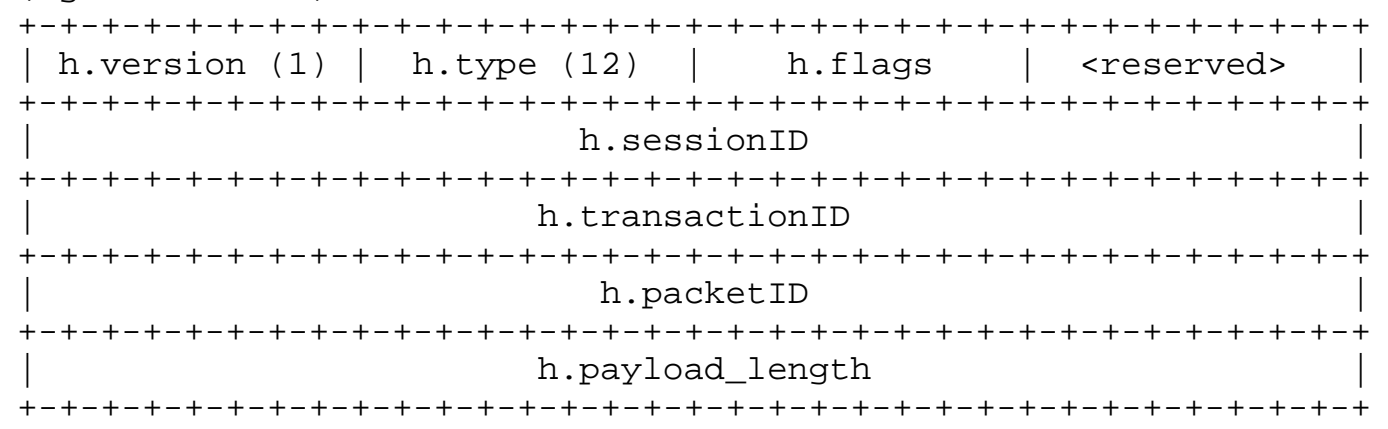

(n. context) OPTIONAL

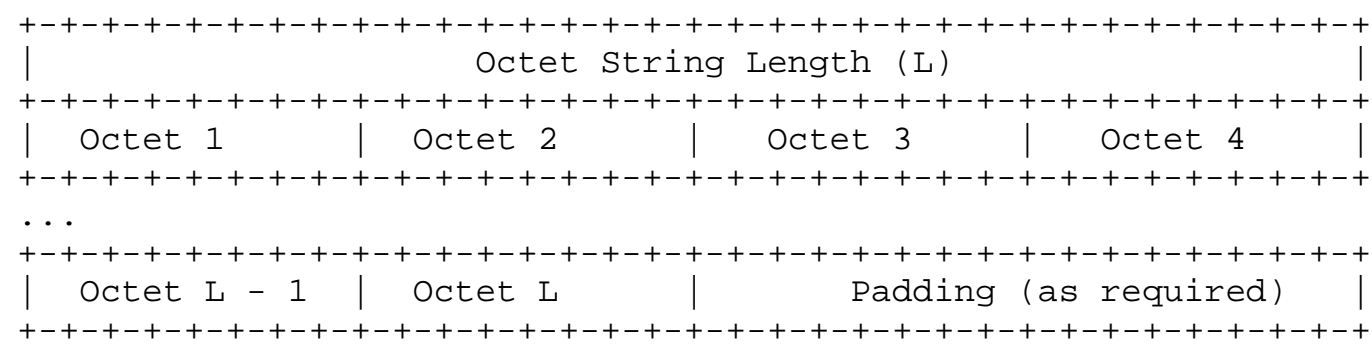

$(\mathrm{n} \cdot \mathrm{vb})$

-.

An agentx-Notify-PDU contains the following fields:

n. context

An optional non-default context.

$\mathrm{n} \cdot \mathrm{vb}$

A VarBindList whose contents define the actual PDU to be sent. This memo places the following restrictions on its contents:

- If the subagent supplies sysuptime.0, it must be present as the first varbind. 
- snmpTrapoid.0 must be present, as the second varbind if sysUpTime.0 was supplied, as the first if it was not.

\subsubsection{The agentx-Ping-PDU}

The agentx-Ping-PDU is sent by a subagent to the master agent to monitor the master agent's ability to receive and send AgentX PDUs over their AgentX session.

(AgentX header)

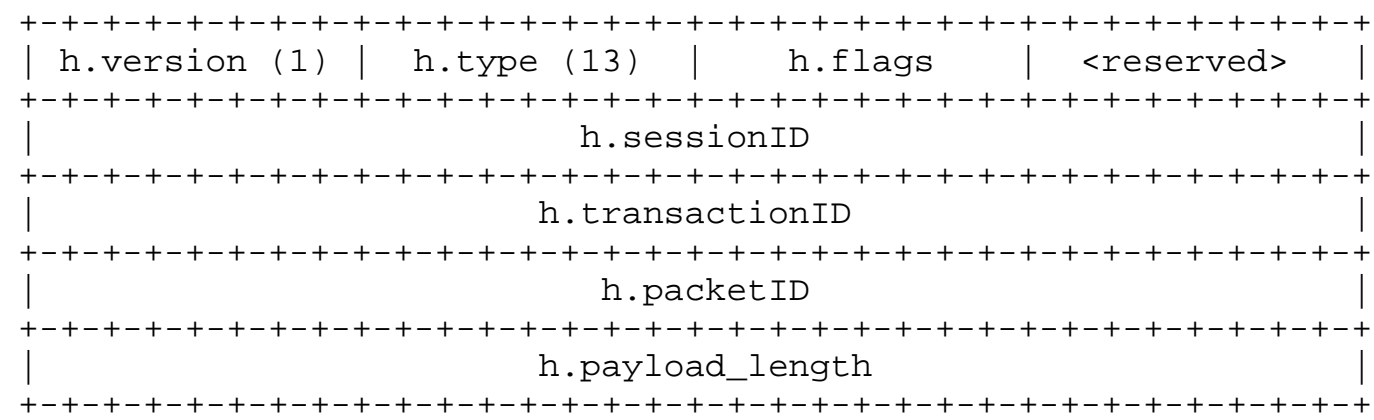

(p.context) OPTIONAL

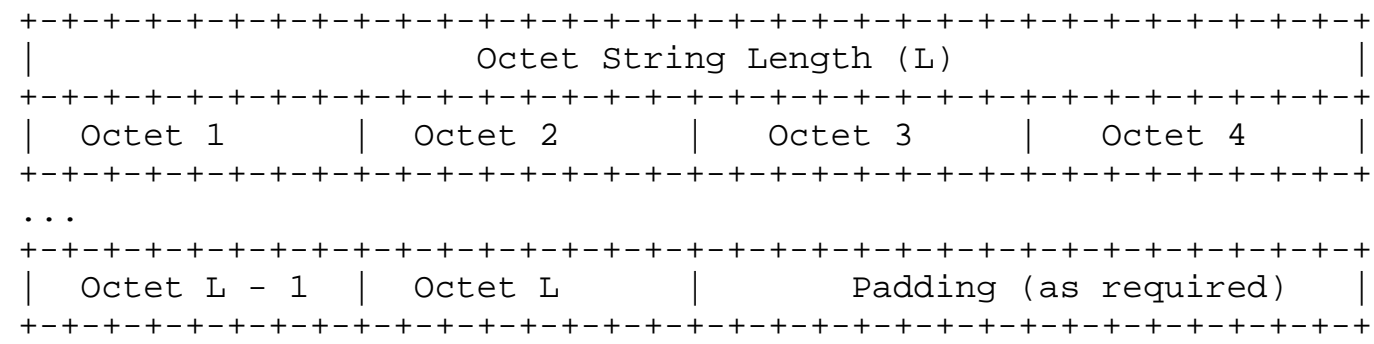

An agentx-Ping-PDU may contain the following field:

p.context

An optional non-default context.

Using p.context a subagent can retrieve the sysUpTime value for a specific context, if required.

6.2.12. The agentx-IndexAllocate-PDU

An agentx-IndexAllocate-PDU is sent by a subagent to request allocation of a value for specific index objects. Refer to section 7.1.4.2, "Registering stuff", for suggested usage. 
(AgentX header)

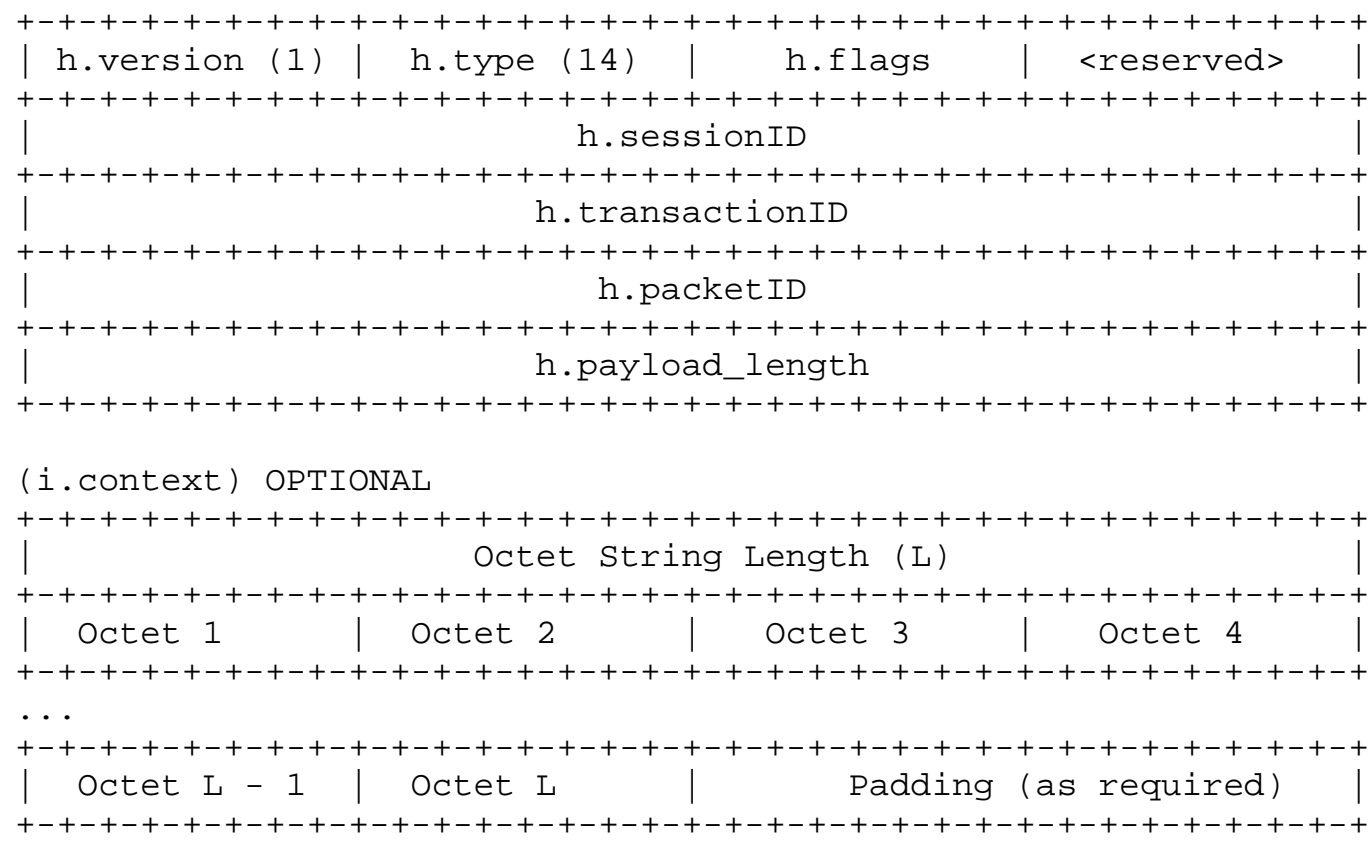

(i.vb)

- .

An agentx-IndexAllocate-PDU contains the following fields:

i. context

An optional non-default context.

i. vb

A VarBindList containing the index names and values requested for allocation.

6.2.13. The agentx-IndexDeallocate-PDU

An agentx-IndexDeallocate-PDU is sent by a subagent to release previously allocated index values. 


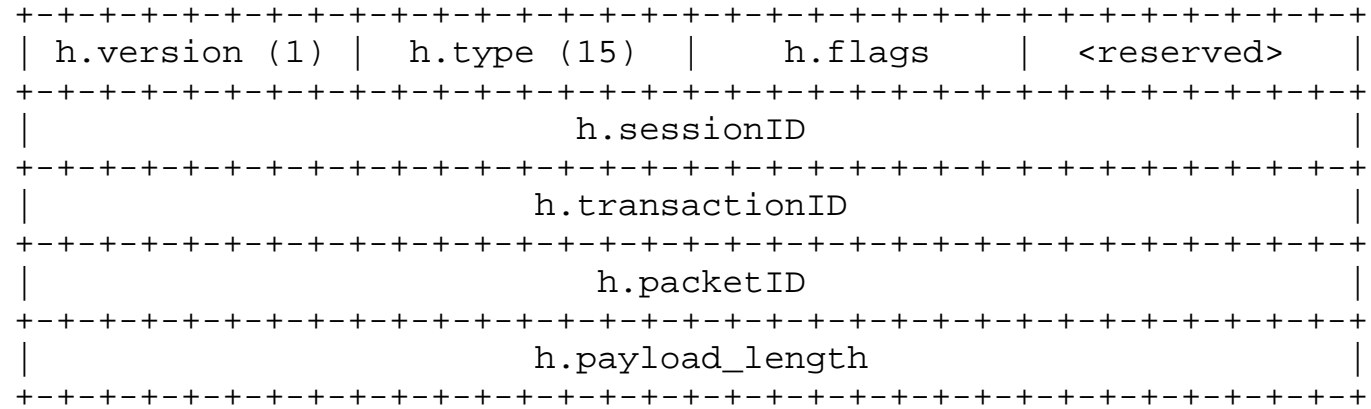

(i.context) OPTIONAL

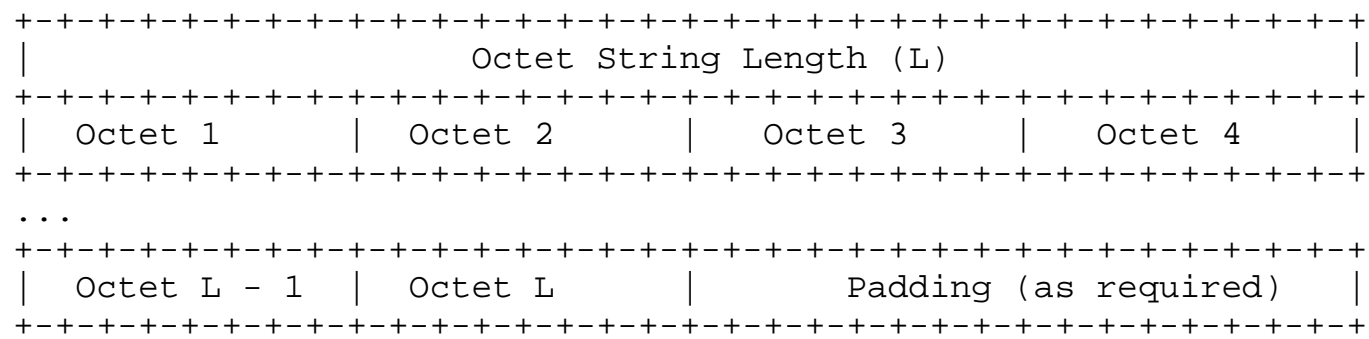

(i.vb)

$\cdots$

An agentx-IndexDeallocate-PDU contains the following fields:

i.context

An optional non-default context.

i. vb

A VarBindList containing the index names and values to be released.

6.2.14. The agentx-AddAgentCaps-PDU

An agentx-AddAgentCaps-PDU is generated by a subagent to inform the master agent of agent capabilities for the specified session. 
(AgentX header)

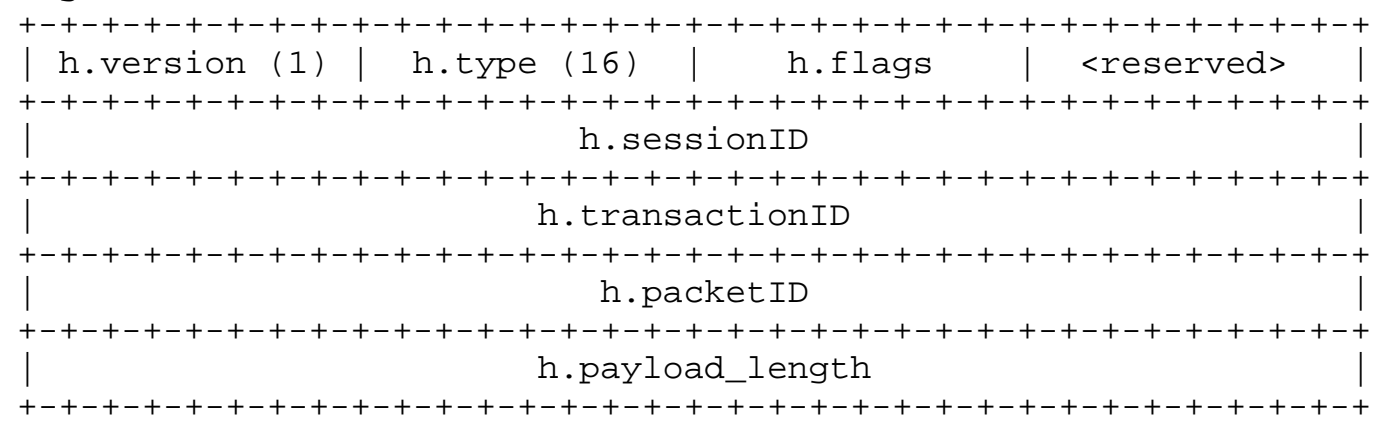

(a.context) (OPTIONAL)

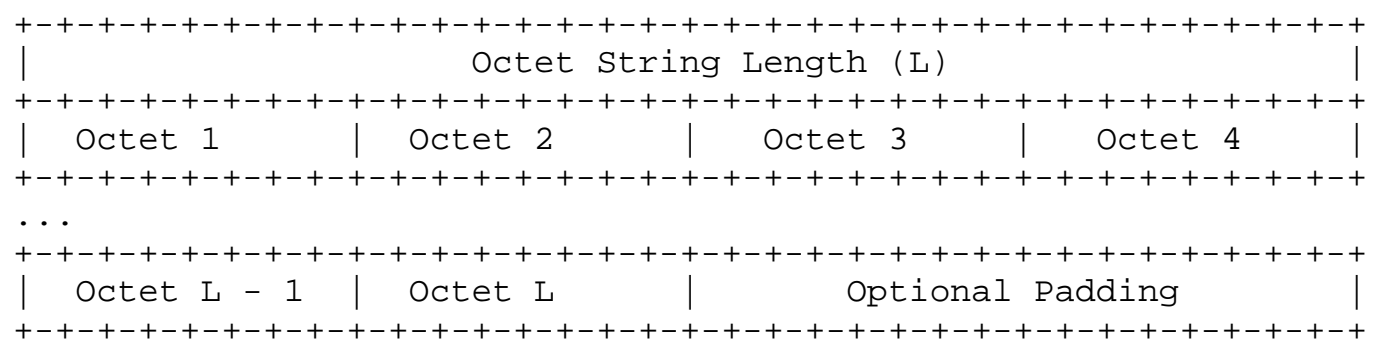

(a.id)

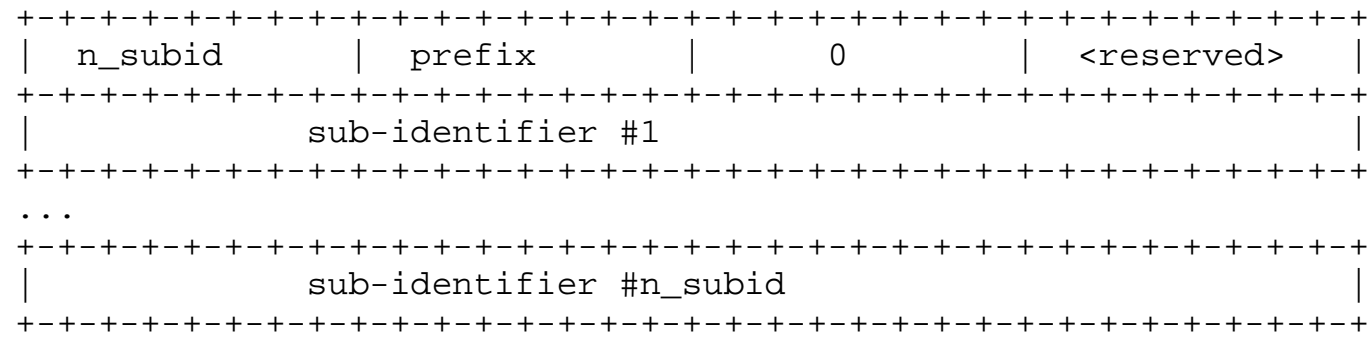

(a.descr)

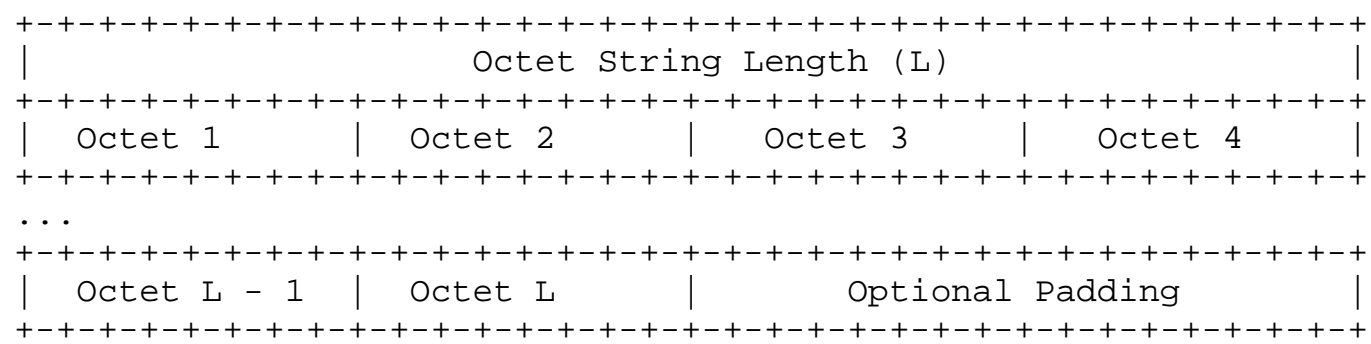


An agentx-AddAgentCaps-PDU contains the following fields:

a. context

An optional non-default context.

a.id

An Object Identifier containing the value of an invocation of the AGENT-CAPABILITIES macro, which the master agent exports as a value of sysORID for the indicated context. (Recall that the value of an invocation of an AGENTCAPABILITIES macro is an object identifier that describes a precise level of support with respect to implemented MIB modules. A more complete discussion of the AGENTCAPABILITIES macro and related sysORID values can be found in section 6 of $\operatorname{STD} 58, \operatorname{RFC} 2580$ [7].)

a.descr

An Octet String containing a Displaystring to be used as the value of sysORDescr corresponding to the sysORID value above.

6.2.15. The agentx-RemoveAgentCaps-PDU

An agentx-RemoveAgentCaps-PDU is generated by a subagent to request that the master agent stop exporting a particular value of sysORID. This value must have previously been advertised by the subagent in an agentx-AddAgentCaps-PDU for this session.

(AgentX header)

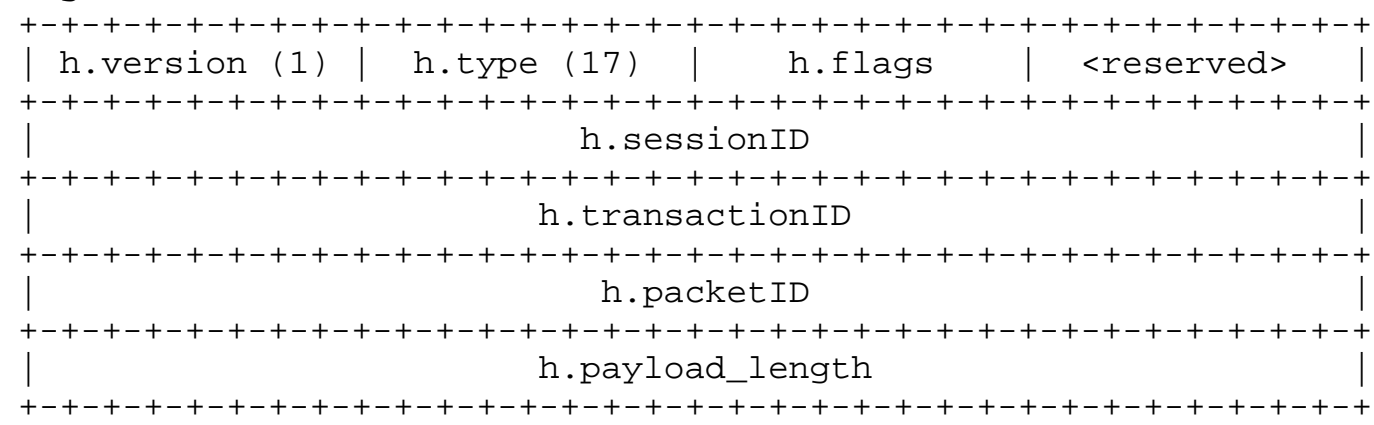

Daniele, et al.

Standards Track

[Page 41] 
(a.context) (OPTIONAL)

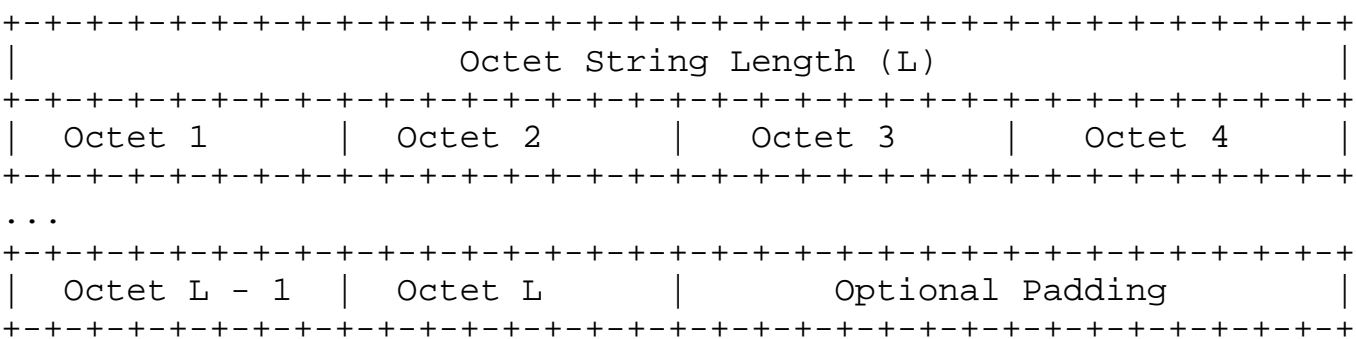

(a.id)

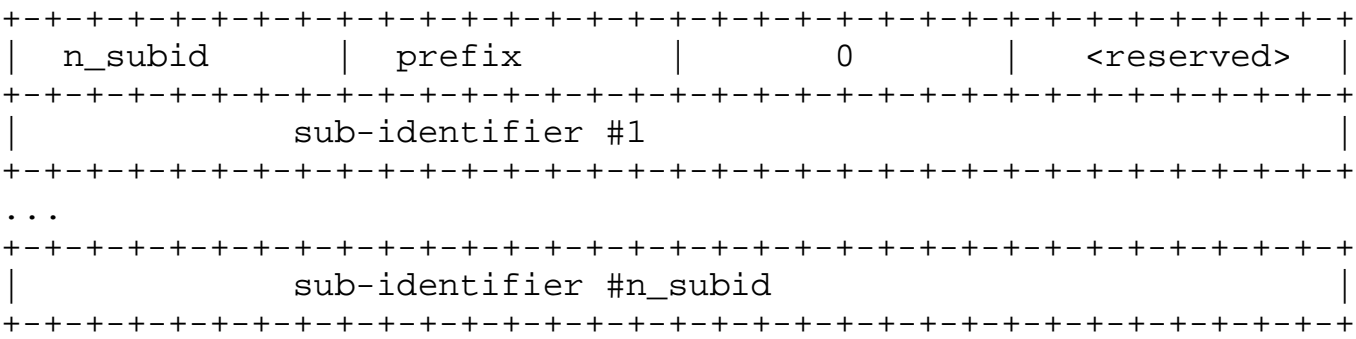

An agentx-RemoveAgentCaps-PDU contains the following fields:

a.context

An optional non-default context.

a.id

An ObjectIdentifier containing the value of sysoRID that should no longer be exported. 
6.2.16. The agentx-Response-PDU

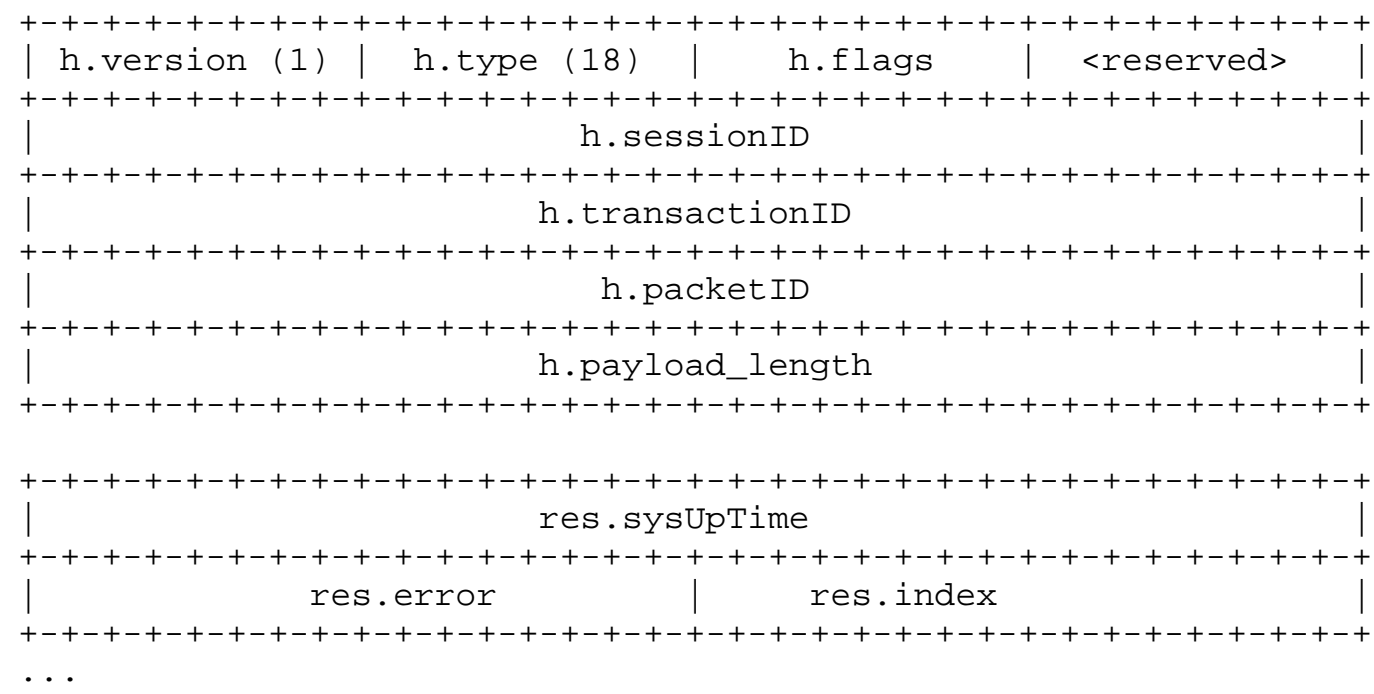

An agentx-Response-PDU contains the following fields:

h. sessionID

If this is a response to an agentx-Open-PDU, then it contains the new and unique sessionID (as assigned by the master agent) for this session.

Otherwise it must be identical to the h.sessionID value in the PDU to which this $\mathrm{PDU}$ is a response.

h.transactionID

Must be identical to the h.transactionID value in the PDU to which this PDU is a response.

In an agentx response PDU from the master agent to the subagent, the value of h.transactionID has no significance and can be ignored by the subagent.

h.packetID

Must be identical to the h.packetID value in the PDU to which this PDU is a response. 
res.sysuptime

This field contains the current value of sysUpTime for the context indicated within the PDU to which this PDU is a response. It is relevant only in agentx response PDUs sent from the master agent to a subagent in response to the set of administrative PDUs listed in section 6.1, "AgentX PDU Header".

In an agentx response PDU from the subagent to the master agent, the value of res.sysupTime has no significance and is ignored by the master agent.

res.error

Indicates error status. Within responses to the set of "administrative" PDU types listed in section 6.1, "AgentX PDU Header", values are limited to the following:

$\begin{array}{ll}\text { noAgentXError } & (0), \\ \text { openFailed } & (256), \\ \text { notopen } & (257), \\ \text { indexWrongType } & (258), \\ \text { indexAlreadyAllocated } & (259), \\ \text { indexNoneAvailable } & (260), \\ \text { indexNotAllocated } & (261), \\ \text { unsupportedContext } & (262), \\ \text { duplicateRegistration } & (263), \\ \text { unknownRegistration } & (264), \\ \text { unknownAgentCaps } & (265), \\ \text { parseError } & (266), \\ \text { requestDenied } & (267), \\ \text { processingError } & (268)\end{array}$

Within responses to the set of "SNMP request processing" PDU types listed in section 6.1, "AgentX PDU Header", values may also include those defined for errors in the SNMPv2 PDU (RFC 1905 [13]).

res.index

In error cases, this is the index of the failed variable binding within a received request PDU. (Note: As explained in section 5.4, "Value Representation", the index values of variable bindings within a variable binding list are 1based.) 
A VarBindList may follow res.index, depending on which Agentx PDU is being responded to. These data are specified in the subsequent elements of procedure.

7. Elements of Procedure

This section describes the actions of protocol entities (master agents and subagents) implementing the Agentx protocol. Note, however, that it is not intended to constrain the internal architecture of any conformant implementation.

The actions of Agentx protocol entities can be broadly categorized under two headings, each of which is described separately:

(1) processing Agentx administrative messages (e.g., registration requests from a subagent to a master agent); and

(2) processing SNMP messages (the coordinated actions of a master agent and one or more subagents in processing, for example, a received SNMP GetRequest-PDU) .

\subsection{Processing Agentx Administrative Messages}

This subsection describes the actions of Agentx protocol entities in processing Agentx administrative messages. Such messages include those involved in establishing and terminating an Agentx session between a subagent and a master agent, those by which a subagent requests allocation of instance index values, and those by which a subagent communicates to a master agent which MIB regions it supports.

Processing is defined specifically for each PDU type in its own section. For the master agent, many of these PDU types require the same initial processing steps. This common processing is defined here, and referenced as needed in the PDU type-specific descriptions.

Common Processing:

The master agent initially processes a received AgentX PDU as follows:

1) An agentx-Response-PDU is created, res.sysUpTime is set to the value of sysUpTime.0 for the default context, res.error is set to 'noAgentxerror', and res.index is set to 0 .

2) If the received PDU cannot be parsed, res.error is set to' parseError'. Examples of a parse error are: 
- PDU length (h.payload) too short to contain current construct (Object Identifier header indicates more subidentifiers, VarBind v.type indicates data follows, etc)

- An unrecognized value is encountered for h.type, v.type, etc.

3) Otherwise, if h.sessionID does not correspond to a currently established session with this subagent, res.error is set to 'notopen'.

4) Otherwise, if the NON_DEFAULT_CONTEXT bit is set and the master agent does not support the indicated context, res.error is set to 'unsupportedContext'. If the master agent does support the indicated context, the value of res.sysupTime is set to the value of sysuptime.0 for that context.

Note: Non-default contexts might be added on the fly by the master agent, or the master agent might require such non-default contexts to be pre-configured. The choice is implementation-specific.

5) If resources cannot be allocated or some other condition prevents processing, res.error is set to 'processingerror'.

6) At this point, if res.error is not 'noAgentXerror', the received PDU is not processed further. If the received PDU's header was successfully parsed, the AgentX-Response-PDU is sent in reply. If the received PDU contained a VarBindList which was successfully parsed, the AgentX-Response-PDU contains the identical VarBindList. If the received PDU's header was not successfully parsed or for some other reason the master agent cannot send a reply, processing is complete.

\subsubsection{Processing the agentx-Open-PDU}

When the master agent receives an agentx-Open-PDU, it processes it as follows:

1) An agentx-Response-PDU is created, res.sysUpTime is set to the value of sysUpTime.0 for the default context, res.error is set to 'noAgentXerror', and res.index is set to 0 .

2) If the received PDU cannot be parsed, res.error is set to 'parseError'.

3) Otherwise, if the master agent is unable to open an Agentx session for any reason, res.error is set to 'openfailed'. 
4) Otherwise: The master agent assigns a sessionID to the new session and puts the value in the h.sessionID field of the agentx-Response-PDU. This value must be unique among all existing open sessions.

The master agent retains session-specific information from the PDU for this session:

- The NETWORK_BYTE_ORDER value in h.flags is retained. All subsequent AgentX protocol operations initiated by the master agent for this session must use this byte ordering and set this bit accordingly.

The subagent typically sets this bit to correspond to its native byte ordering, and typically does not vary byte ordering for an initiated session. The master agent must be able to decode each PDU according to the h.flag NETWORK_BYTE_ORDER bit in the PDU, but does not need to toggle its retained value for the session if the subagent varies its byte ordering.

- The o.timeout value is used in calculating response timeout conditions for this session. This field is also referenced in the AgentX MIB (a work-in-progress) by the agentxSessionTimeout object.

- The o.id and o.descr fields are used for informational purposes. These two fields are also referenced in the Agentx MIB (a work-in-progress) by the agentxSessionobjectID object, and by the agentxisessionDescr object.

5) The agentx-Response-PDU is sent with the res.error field indicating the result of the session initiation.

If processing was successful, an Agentx session is considered established between the master agent and the subagent. An Agentx session is a distinct channel for the exchange of Agentx protocol messages between a master agent and one subagent, qualified by the session-specific attributes listed in 4) above. Agentx session establishment is initiated by the subagent. An Agentx session can be terminated by either the master agent or the subagent.

\subsubsection{Processing the agentx-IndexAllocate-PDU}

When the master agent receives an agentx-IndexAllocate-PDU, it performs the common processing described in section 7.1, "Processing AgentX Administrative Messages". If as a result res.error is 'noAgentXerror', processing continues as follows: 
1) Each VarBind in the VarBindList is processed until either all are successful, or one fails. If any VarBind fails, the agentxResponse-PDU is sent in reply containing the original VarBindList, with res.index set to indicate the failed VarBind, and with res.error set as described subsequently. All other VarBinds are ignored; no index values are allocated.

VarBinds are processed as follows:

- V.name is the OID prefix of the MIB OBJECT-TYPE for which a value is to be allocated.

- v.type is the syntax of the MIB OBJECT-TYPE for which a value is to be allocated.

- v.data indicates the specific index value requested. If the NEW_INDEX or the ANY_INDEX bit is set, the actual value in v.data is ignored and an appropriate index value is generated.

a) If there are no currently allocated index values for $v$.name in the indicated context, and v.type does not correspond to a valid index type value, the VarBind fails and res.error is set to 'indexWrongType'.

b) If there are currently allocated index values for $v$. name in the indicated context, but the syntax of those values does not match v.type, the VarBind fails and res.error is set to 'indexWrongType'.

c) Otherwise, if both the NEW_INDEX and ANY_INDEX bits are clear, allocation of a specific index value is being requested. If the requested index is already allocated for $v$.name in the indicated context, the VarBind fails and res.error is set to 'indexAlreadyAllocated'.

d) Otherwise, if the NEW_INDEX bit is set, the master agent should generate the next available index value for v.name in the indicated context, with the constraint that this value must not have been allocated (even if subsequently released) to any subagent since the last re-initialization of the master agent. If no such value can be generated, the VarBind fails and res.error is set to 'indexNoneAvailable'.

e) Otherwise, if the ANY_INDEX bit is set, the master agent should generate an index value for $v$.name in the indicated context, with the constraint that this value is not currently allocated to any subagent. If no such value can be generated, then the VarBind fails and res.error is set to 'indexNoneAvailable'. 
2) If all VarBinds are processed successfully, the agentx-ResponsePDU is sent in reply with res.error set to 'noAgentXError'. A VarBindList is included that is identical to the one sent in the agentx-IndexAllocate-PDU, except that VarBinds requesting a NEW_INDEX or ANY_INDEX value are generated with an appropriate value.

See section 7.1.4.2, "Registering Stuff" for more information on how subagents should perform index allocations.

\subsubsection{Processing the agentx-IndexDeallocate-PDU}

When the master agent receives an agentx-IndexDeallocate-PDU, it performs the common processing described in section 7.1, "Processing AgentX Administrative Messages". If as a result res.error is 'noAgentXerror', processing continues as follows:

1) Each VarBind in the VarBindList is processed until either all are successful, or one fails. If any VarBind fails, the agentxResponse-PDU is sent in reply, containing the original VarBindList, with res.index set to indicate the failed VarBind, and with res.error set as described subsequently. All other VarBinds are ignored; no index values are released.

VarBinds are processed as follows:

- v.name is the name of the index for which a value is to be released

- v.type is the syntax of the index object

- v.data indicates the specific index value to be released. The NEW_INDEX and ANY_INDEX bits are ignored.

a) If the index value for the named index is not currently allocated to this session, the VarBind fails and res.error is set to 'indexNotAllocated'.

2) If all VarBinds are processed successfully, res.error is set to 'noAgentXError' and the agentx-Response-PDU is sent. A VarBindList is included which is identical to the one sent in the agentx-IndexDeallocate-PDU.

All released index values are now available, and may be used in response to subsequent allocation requests for ANY_INDEX values and in response to subsequent allocation requests for the particular index value. 


\subsubsection{Processing the agentx-Register-PDU}

When the master agent receives an agentx-Register-PDU, it performs the common processing described in section 7.1, "Processing AgentX Administrative Messages". If as a result res.error is 'noAgentXerror', processing continues as follows:

If any of the union of subtrees defined by this MIB region is exactly the same as any subtree defined by a MIB region currently registered within the indicated context, those subtrees are termed "duplicate subtrees".

If any of the union of subtrees defined by this MIB region overlaps, or is itself overlapped by, any subtree defined by a MIB region currently registered within the indicated context, those subtrees are termed "overlapping subtrees".

1) If this registration would result in duplicate subtrees registered with the same value of r.priority, the request fails and an agentx-Response-PDU is returned with res.error set to 'duplicateRegistration'.

2) Otherwise, if the master agent does not wish to permit this registration for implementation-specific reasons, the request fails and an agentx-Response-PDU is returned with res.error set to 'requestDenied'.

3) Otherwise, the agentx-Response-PDU is returned with res.error set to 'noAgentxerror'.

The master agent adds this MIB region to its registration data store for the indicated context, to be considered during the dispatching phase for subsequently received SNMP protocol messages.

7.1.4.1. Handling Duplicate and Overlapping Subtrees

As a result of this registration algorithm there are likely to be duplicate and/or overlapping subtrees within the registration data store of the master agent. Whenever the master agent's dispatching algorithm (see section 7.2.1, "Dispatching AgentX PDUs") determines that there are multiple subtrees that could potentially contain the same MIB object instances, the master agent selects one to use, termed the 'authoritative region', as follows: 
1) Choose the one whose original agentx-Register-PDU r.subtree contained the most subids, i.e., the most specific r.subtree. Note: The presence or absence of a range subid has no bearing on how "specific" one object identifier is compared to another.

2) If still ambiguous, there were duplicate subtrees. Choose the one whose original agentx-Register-PDU specified the smaller value of r.priority.

\subsubsection{Registering Stuff}

This section describes more fully how Agentx subagents use the agentx-IndexAllocate-PDU and agentx-Register-PDU to achieve desired configurations.

\subsection{Registration Priority}

The r.priority field in the agentx-Register-PDU is intended to be manipulated by human administrators to achieve a desired subagent configuration. Typically this would be needed where a legacy application registers a specific subtree, and a different (configurable) application may need to become authoritative for the identical subtree.

The result of this configuration (the same subtree registered on different sessions with different priorities) is that the session using the better priority (see section 7.1.4.1, "Handling Duplicate and Overlapping Subtrees") will be authoritative. The other session will simply never be dispatched to.

This is useful in the case described above, but is NOT useful in other cases, particularly when subagents share tables indexed by arbitrary values (see below). In general, subagents should register using the default priority (127).

7.1.4.2.2. Index Allocation

Index allocation is a service provided by an Agentx master agent. It provides generic support for sharing MIB conceptual tables among subagents who are assumed to have no knowledge of each other.

The master agent maintains a database of index objects (OIDs), and, for each index, the values that have been allocated for it. It is unaware of what MIB variables (if any) the index objects represent. 
By convention, subagents use the MIB variable listed in the INDEX clause as the index object for which values must be allocated. For tables indexed by multiple variables, values may be allocated for each index (although this is frequently unnecessary; see example 2 below). The subagent may request allocation of
a) a specific index value
b) an index value that is not currently allocated
c) an index value that has never been allocated

The last two alternatives reflect the uniqueness and constancy requirements present in many MIB specifications for arbitrary integer indexes (e.g., ifIndex in the IF-MIB (RFC 2233 [19]), snmpFddiSMTIndex in the FDDI MIB (RFC 1285 [20]), or sysApplInstallpkgIndex in the system Application MIB (RFC 2287 [21])). The need for subagents to share tables using such indexes is the main motivation for index allocation in Agentx.

It is important to note that index allocation and MIB region registration are not coupled in the master agent. The current state of index allocations is not considered when processing registration requests, and the current registry is not considered when processing index allocation requests. (This is mainly to accommodate non-Agentx subagents.)

Agentx subagents should follow the model of "first request allocation of an index, then register the corresponding region". Then a successful index allocation request gives a subagent a good hint (but no guarantee) of what it should be able to register. The registration may fail (with 'duplicateRegistration') because some other subagent session has already registered that row of the table.

The recommended mechanism for subagents to register conceptual rows in a shared table is

1) Successfully allocate an index value.

2) Use that value to fully qualify the MIB region(s), and attempt to register using the default priority.

3) If the registration fails with 'duplicateRegistration' deallocate the previously allocated index value(s) for this row and go to step 1). 
Note that index allocation is necessary only when the index in question is an arbitrary value, and hence the subagent has no other reasonable way to determine which index values to use. When index values have intrinsic meaning it is not expected that subagents will allocate their index values.

For example, RFC 1514's table of running software processes (hrSWRunTable) is indexed by the system's native process identifier (pid). A subagent implementing the row of hrSWRunTable corresponding to its own process would simply register the region defining that row's object instances without allocating index values.

\subsection{Examples}

Example 1:

A subagent implements an interface, and wishes to register a single row of the RFC 2233 iftable. It requests an allocation for the index object "ifIndex", for a value that has never been allocated (since ifIndex values must be unique). The master agent returns the value "7".

The subagent now attempts to register row 7 of iftable, by specifying a MIB region in the agentx-Register-PDU of 1.3.6.1.2.1.2.2.1.[1-22].7. If the registration succeeds, no further processing is required. The master agent will dispatch to this subagent correctly.

If the registration failed with 'duplicateRegistration', the subagent should deallocate the failed index, request allocation of a new index $i$, and attempt to register ifTable.[1-22].i, until successful.

Example 2:

This same subagent wishes to register ipNetToMediaTable rows corresponding to its interface (ifIndex i). Due to the structure of this table, no further index allocation need be done. The subagent can register the MIB region ipNetToMediaTable.[1-4].i, It is claiming responsibility for all rows of the table whose value of ipNetToMediaIfIndex is i. 
Example 3:

A network device consists of a set of processors, each of which accepts network connections for a unique set of IP addresses. Further, each processor contains a subagent that implements tcpConnTable. In order to represent tcpconntable for the entire managed device, the subagents need to share tcpconnTable.

In this case, no index allocation need be done at all. Each subagent can register a MIB region of tcpConnTable.[1-5].a.b.c.d, where a.b.c.d represents an unique IP address of the individual processor.

Each subagent is claiming responsibility for the region of tcpconntable where the value of tcpConnLocalAddress is a.b.c.d.

Example 4:

The Application Management MIB (RFC 2564 [22]) uses two objects to index several tables. A partial description of them is:

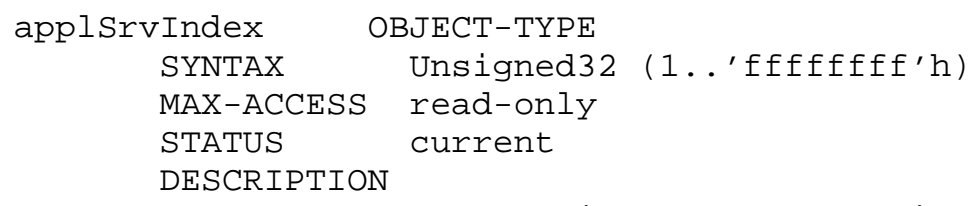

"An applSrvIndex is the system-unique identifier of an instance of a service. The value is unique not only across all instances of a given service, but also across all services in a system."

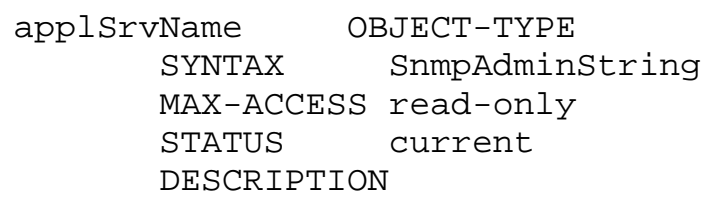


Since applSrvindex is an arbitrary value, it would be reasonable for subagents to allocate values for this index. applsrvName however has intrinsic meaning and any values a subagent would use should be known a priori, hence it is not reasonable for subagents to allocate values of this index.

7.1.5. Processing the agentx-Unregister-PDU

When the master agent receives an agentx-Unregister-PDU, it performs the common processing described in section 7.1, "Processing AgentX Administrative Messages". If as a result res.error is. noAgentXError', processing continues as follows:

1) If u.subtree, u.priority, u.range_subid (and if u.range_subid is not 0 , u.upper_bound), and the indicated context do not match an existing registration made during this session, the agentxResponse-PDU is returned with res.error set to' unknownRegistration'.

2) Otherwise, the agentx-Response-PDU is sent in reply with res.error set to 'noAgentXError', and the previous registration is removed from the registration data store.

7.1.6. Processing the agentx-AddAgentCaps-PDU

When the master agent receives an agentx-AddAgentCaps-PDU, it performs the common processing described in section 7.1, "Processing Agentx Administrative Messages". If as a result res.error is. noAgentXerror', processing continues as follows:

1) The master agent adds this agent capabilities information to the sysorTable for the indicated context. An agentx-Response-PDU is sent in reply with res.error set to 'noAgentxerror'.

7.1.7. Processing the agentx-RemoveAgentCaps-PDU

When the master agent receives an agentx-RemoveAgentCaps-PDU, it performs the common processing described in section 7.1, "Processing Agentx Administrative Messages". If as a result res.error is 'noAgentXerror', processing continues as follows:

1) If the combination of a.id and the optional a.context does not represent a sysORTable entry that was added by this subagent during this session, the agentx-Response-PDU is returned with res.error set to 'unknownAgentCaps'. 
2) Otherwise the master agent deletes the corresponding sysorTable entry and sends in reply the agentx-Response-PDU, with res.error set to 'noAgentXError'.

\subsubsection{Processing the agentx-Close-PDU}

When the master agent receives an agentx-Close-PDU, it performs the common processing described in section 7.1, "Processing AgentX Administrative Messages", with the exception that step 4) is not performed since the agentx-Close-PDU does may not contain a context field. If as a result res.error is 'noAgentXError', processing continues as follows:

1) The master agent closes the AgentX session as described below, and sends in reply the agentx-Response-PDU with res.error set to 'noAgentXerror' :

- All MIB regions that have been registered during this session are unregistered, as described in section 7.1.5, "Processing the agentx-Unregister-PDU" .

- All index values allocated during this session are freed, as described in section 7.1.3, "Processing the agentxIndexDeallocate-PDU".

- All sysORID values that were registered during this session are removed, as described in section 7.1.7, "Processing the agentx-RemoveAgentCaps-PDU" .

The master agent does not maintain state for closed sessions. If a subagent wishes to re-establish a session after it has been closed, it needs to re-register MIB regions, agent capabilities, etc.

\subsubsection{Detecting Connection Loss}

If a master agent is able to detect (from the underlying transport) that a subagent cannot receive AgentX PDUs, it should close all affected AgentX sessions as described in section 7.1.8, "Processing the agentx-Close-PDU", step 1).

7.1.10. Processing the agentx-Notify-PDU

A subagent sending SNMPv1 trap information must map this into (minimally) a value of snmpTrapoID.0, as described in 3.1 .2 of RFC 1908 [24]. 
When the master agent receives an agentx-Notify-PDU, it performs the common processing described in section 7.1, "Processing AgentX Administrative Messages". If as a result res.error is 'noAgentXerror', processing continues as follows:

1) If the first VarBind is sysUpTime.0;

(a) if the second VarBind is not snmpTrapoID.0, res.error is set to 'processingError' and res.index to 2

(b) otherwise these two VarBinds are used as the first two VarBinds within the generated notification.

2) If the first VarBind is not sysUpTime.0;

(a) if the first VarBind is not snmpTrapoID.0, res.error is set to 'processingError' and res.index to 1

(b) otherwise this VarBind is used for snmpTrapoID.0 within the generated notification, and the master agent uses the current value of sysUpTime.0 for the indicated context as sysUpTime.0 within the notification.

3) An agentx-Response-PDU is sent containing the original

VarBindList, and with res.error and res.index set as described above. If res.error is 'noAgentXError', notifications are sent according to the implementation-specific configuration of the master agent. If SNMPV1 Trap PDUs are generated, the recommended mapping is as described in RFC 2089 [25]. If res.error indicates an error in processing, no notifications are generated.

Note that the master agent's successful response indicates the agentx-Notify-PDU was received and validated. It does not indicate that any particular notifications were actually generated or received by notification targets.

7.1.11. Processing the agentx-Ping-PDU

When the master agent receives an agentx-Ping-PDU, it performs the common processing described in section 7.1, "Processing AgentX Administrative Messages". If as a result res.error is ' noAgentXerror', processing continues as follows: 
1) An agentx-Response-PDU is sent in reply.

If a subagent does not receive a response to its pings, or if it is able to detect (from the underlying transport) that the master agent is not able to receive Agentx messages, then it eventually must initiate a new Agentx session, re-register its MIB regions, etc.

\subsection{Processing Received SNMP Protocol Messages}

When an SNMP GetRequest, GetNextRequest, GetBulkRequest, or SetRequest protocol message is received by the master agent, the master agent applies its access control policy.

In particular, for SNMPV1 or SNMPV2c protocol messages, the master agent applies the Elements of Procedure defined in section 4.1 of STD 15, RFC 1157 [8] that apply to receiving entities. For SNMPv3, the master agent applies an Access Control Model, possibly the View-based Access Control Model (see RFC 2575 [15]), as described in section 3.1 .2 and section 4.3 of RFC 2571 [1].

For SNMPV1 and SNMPV2c, the master agent uses the community string as an index into a local repository of configuration information that may include community profiles or more complex context information. For SNMPv3, the master agent uses the SNMP Context (see section 3.3 .1 of RFC 2571 [1]) for these purposes.

If application of the access control policy results in a valid SNMP request $\mathrm{PDU}$, then an SNMP Response-PDU is constructed from information gathered in the exchange of Agentx PDUs between the master agent and one or more subagents. Upon receipt and initial validation of an SNMP request PDU, a master agent uses the procedures described below to dispatch Agentx PDUs to the proper subagents, marshal the subagent responses, and construct an SNMP response PDU.

\subsubsection{Dispatching AgentX PDUs}

Upon receipt and initial validation of an SNMP request PDU, a master agent uses the procedures described below to dispatch AgentX PDUs to the proper subagents.

General Rules of Procedure

While processing a particular SNMP request, the master agent may send one or more Agentx PDUs on one or more subagent sessions. The following rules of procedure apply in general to the Agentx master agent. PDU-specific rules are listed in the applicable sections. 
1) Honoring the registry

Because Agentx supports registration of duplicate and overlapping regions, it is possible for the master agent to obtain a value for a requested varbind from within multiple registered MIB regions.

The master agent must ensure that the value (or exception) actually returned in the SNMP response PDU is taken from the authoritative region (as defined in section 7.1.4.1, "Handling Duplicate and Overlapping Subtrees").

2) GetNext and GetBulk Processing

The master agent may choose to send agentx-Get-PDUs while servicing an SNMP GetNextRequest-PDU. The master agent may choose to send agentx-Get-PDUs or agentx-GetNext-PDUs while servicing an SNMP GetBulkRequest-PDU. One possible reason for this would be if the current iteration has targeted instance-level registrations.

The master agent may choose to "scope" the possible instances returned by a subagent by specifying an ending OID in the SearchRange. If such scoping is used, typically the ending OID would be the first lexicographical successor to the target region that was registered on a session other than the target session. Regardless of this choice, rule (1) must be obeyed.

The master agent may require multiple request-response iterations on the same subagent session, to determine the final value of all requested variables.

All AgentX PDUs sent on the session while processing a given SNMP request must contain identical values of transactionID. Each different SNMP request processed by the master agent must present a unique value of transactionID (within the limits of the 32-bit field) to the session.

3) Number and order of variables sent per AgentX PDU

For Get/GetNext/GetBulk operations, at any stage of the possibly iterative process, the master agent may need to dispatch several SearchRanges to a particular subagent session. The master agent may send one, some, or all of the SearchRanges in a single Agentx PDU .

The master agent must ensure that the correct contents and ordering of the VarBindList in the SNMP Response-PDU are maintained. 
The following rules govern the number of VarBinds in a given AgentX PDU:

a) The subagent must support processing of AgentX PDUs with multiple VarBinds.

b) When processing an SNMP set request, the master agent must send all of the VarBinds applicable to a particular subagent session in a single agentx-TestSet-PDU.

c) When processing an SNMP Get, GetNext, or GetBulk request, the master agent may send a single AgentX PDU on the session with all applicable VarBinds, or multiple PDUs with single VarBinds, or something in between those extremes. The determination of which method to use in a particular case is implementation-specific.

4) Timeout Values

The master agent chooses a timeout value for each MIB region being queried, which is

a) the value specified during registration of the MIB region, if it was non-zero

b) otherwise, the value specified during establishment of the session in which this region was subsequently registered, if that value was non-zero

c) otherwise, or, if the specified value is not practical, the master agent's implementaton-specific default value

When an AgentX PDU that references multiple MIB regions is dispatched, the timeout value used for the PDU is the maximum value of the timeouts so determined for each of the referenced MIB regions.

5) Context

If the master agent has determined that a specific non-default context is associated with the SNMP request PDU, that context is encoded into the Agentx PDU's context field and the NON_DEFAULT_CONTEXT bit is set in h.flags.

Otherwise, no context Octet String is added to the PDU, and the NON_DEFAULT_CONTEXT bit is cleared. 


\subsubsection{1. agentx-Get-PDU}

Each variable binding in the SNMP request PDU is processed as follows :

(1) Identify the target MIB region.

Within a lexicographically ordered set of registered MIB regions, valid for the indicated context, locate the authoritative region (according to section 7.1.4.1, "Handling Duplicate and Overlapping subtrees") that contains the binding's name.

(2) If no such region exists, the variable binding is not processed further, and its value is set to 'nosuchobject'.

(3) Identify the subagent session in which this region was registered, termed the target session.

(4) If this is the first variable binding to be dispatched over the target session in a request-response exchange entailed in the processing of this management request:

- Create an agentx-Get-PDU for this session, with the header fields initialized as described above (see section 6.1, "AgentX PDU Header").

(5) Add a SearchRange to the end of the target session's PDU for this variable binding.

- The variable binding's name is encoded into the starting OID.

- The ending OID is encoded as null.

7.2.1.2. agentx-GetNext-PDU

Each variable binding in the SNMP request PDU is processed as follows:

(1) Identify the target MIB region.

Within a lexicographically ordered set of registered MIB regions, valid for the indicated context, locate the authoritative region (according to section 7.1.4.1, "Handling Duplicate and Overlapping subtrees") that

a) contains the variable binding's name and is not a fully qualified instance, or 
b) is the first lexicographical successor to the variable binding's name.

(2) If no such region exists, the variable binding is not processed further, and its value is set to 'endofMibView'.

(3) Identify the subagent session in which this region was registered, termed the target session.

(4) If this is the first variable binding to be dispatched over the target session in a request-response exchange entailed in the processing of this management request:

- Create an agentx-GetNext-PDU for the session, with the header fields initialized as described above (see section 6.1, "AgentX PDU Header").

(5) Add a SearchRange to the end of the target session's agentxGetNext-PDU for this variable binding.

- if (1a) applies, the variable binding's name is encoded into the starting OID, and the OID's "include" field is set to 0 .

- if (1b) applies, the target region's r.subtree is encoded into the starting OID, and its "include" field is set to 1 . (This is the recommended method. An implementation may choose to use a starting OID value that precedes r.subtree, in which case the include bit must be 0 . A starting OID value that succeeds r.subtree is not permitted.)

- the Ending OID for the SearchRange is encoded to be either NULL, or a value that lexicographically succeeds the starting OID. This is an implementation-specific choice depending on how the master agent wishes to "scope" the possible returned instances.

\subsubsection{3. agentx-GetBulk-PDU}

(Note: The outline of the following procedure is based closely on section 4.2.3, "The GetBulkRequest-PDU" of RFC 1905 [13]. Please refer to it for details on the format of the SNMP GetBulkRequest-PDU itself.) 
Each variable binding in the request PDU is processed as follows:

(1) Identify the authoritative target region and target session, exactly as described for the agentx-GetNext-PDU (see section 7.2.1.2, "agentx-GetNext-PDU") .

(2) If this is the first variable binding to be dispatched over the target session in a request-response exchange entailed in the processing of this management request:

- Create an agentx-GetBulk-PDU for the session, with the header fields initialized as described above (see section 6.1, "AgentX PDU Header").

(3) Add a SearchRange to the end of the target session's agentxGetBulk-PDU for this variable binding, as described for the agentx-GetNext-PDU. If the variable binding was a non-repeater in the original request $\mathrm{PDU}$, it must be a non-repeater in the agentx-GetBulk-PDU.

The value of $g . m a x$ repetitions in the agentx-GetBulk-PDU may be less than (but not greater than) the value in the original request PDU.

The master agent may make such alterations due to simple sanity checking, optimizations for the current iteration based on the registry, the maximum possible size of a potential Response-PDU, known constraints of the Agentx transport, or any other

implementation-specific constraint.

\subsubsection{4. agentx-TestSet-PDU}

Agentx employs test-commit-undo-cleanup phases to achieve "as if simultaneous" semantics of the SNMP setRequest-PDU within the extensible agent. The initial phase involves the agentx-TestSet-PDU.

Each variable binding in the SNMP request PDU is processed in order, as follows:

(1) Identify the target MIB region and target session exactly as described in section 7.2.1.1, "agentx-Get-PDU", step 1).

Within a lexicographically ordered set of OID ranges, valid for the indicated context, locate the authoritative range that contains the variable binding's name.

(2) If no such target region exists, this variable binding fails with an error of 'notWritable'. Processing is complete for this request. 
(3) If this is the first variable binding to be dispatched over the target session in a request-response exchange entailed in the processing of this management request:

- create an agentx-TestSet-PDU for the session, with the header fields initialized as described above (see section 6.1, "AgentX PDU Header").

(4) Add a VarBind to the end of the target session's PDU for this variable binding, as described in section 5.4, "Value Representation".

Note that all VarBinds applicable to a given session must be sent in a single agentx-Testset-PDU.

\subsubsection{Dispatch}

A timeout value is calculated for each PDU to be sent, which is the maximum value of the timeouts determined for each of the PDU' $s$ SearchRanges (as described above in section 7.2.1, "Dispatching AgentX PDUs", item 4). Each pending PDU is mapped (via its h.sessionID value) to a particular transport domain/endpoint, as described in section 8 (Transport Mappings).

\subsubsection{Subagent Processing}

A subagent initially processes a received AgentX PDU as follows:

- If the received PDU is an agentx-Response-PDU:

1) If there are any errors parsing or interpreting the PDU, it is silently dropped.

2) Otherwise the response is matched to the original request via h.packetID, and handled in an implementation-specific manner. For example, if this response indicates an error attempting to register a MIB region, the subagent may wish to register a different region, or log an error and halt, etc.

- If the received PDU is any other type:

1) an agentx-Response-PDU is created whose header fields are identical to the received request $\mathrm{PDU}$ except that $\mathrm{h}$.type is set to Response, res.error to 'noError', res.index to 0 , and the VarBindList to null.

2) If the received PDU cannot be parsed, res.error is set to 'parseError'. 
3) Otherwise, if h.sessionID does not correspond to a currently established session, res.error is set to 'notopen'.

4) At this point, if res.error is not 'noError', the received PDU is not processed further. If the received PDU's header was successfully parsed, the AgentX-Response-PDU is sent in reply. If the received PDU's header was not successfully parsed or for some other reason the subagent cannot send a reply, processing is complete.

7.2.3. Subagent Processing of agentx-Get, GetNext, GetBulk-PDUs

A conformant Agentx subagent must support the agentx-Get, -GetNext, and -GetBulk PDUs, and must support multiple variables being supplied in each PDU.

When a subagent receives an agentx-Get-, GetNext-, or GetBulk-PDU, it performs the indicated management operations and returns an agentxResponse-PDU.

Each SearchRange in the request PDU's SearchRangelist is processed as described below, and a VarBind is added in the corresponding location of the agentx-Response-PDU's VarbindList. If processing should fail for any reason not described below, res.error is set to 'genErr', res.index to the index of the failed SearchRange, the VarBindList is reset to null, and this agentx-Response-PDU is returned to the master agent.

7.2.3.1. Subagent Processing of the agentx-Get-PDU

Upon the subagent's receipt of an agentx-Get-PDU, each SearchRange in the request is processed as follows:

(1) The starting OID is copied to v.name.

(2) If the starting OID exactly matches the name of a variable instantiated by this subagent within the indicated context and session, v.type and v.data are encoded to represent the variable's syntax and value, as described in section 5.4, "Value Representation".

(3) Otherwise, if the starting OID does not match the object identifier prefix of any variable instantiated within the indicated context and session, the VarBind is set to 'noSuchobject', in the manner described in section 5.4, "Value Representation". 
(4) Otherwise, the VarBind is set to 'noSuchInstance' in the manner described in section 5.4, "Value Representation".

7.2.3.2. Subagent Processing of the agentx-GetNext-PDU

Upon the subagent's receipt of an agentx-GetNext-PDU, each SearchRange in the request is processed as follows:

(1) The subagent searches for a variable within the lexicographically ordered list of variable names for all variables it instantiates (without regard to registration of regions) within the indicated context and session, as follows:

- if the "include" field of the starting OID is 0 , the variable's name is the closest lexicographical successor to the starting OID.

- if the "include" field of the starting OID is 1 , the variable's name is either equal to, or the closest lexicographical successor to, the starting OID.

- If the ending OID is not null, the variable's name lexicographically precedes the ending OID.

If a variable is successfully located, v.name is set to that variable's name. v.type and v.data are encoded to represent the variable's syntax and value, as described in section 5.4, "Value Representation".

(2) If the subagent cannot locate an appropriate variable, v.name is set to the starting OID, and the VarBind is set to. endOfMibView', in the manner described in section 5.4, "Value Representation".

7.2.3.3. Subagent Processing of the agentx-GetBulk-PDU

A maximum of $\mathrm{N}+(\mathrm{M} * \mathrm{R})$ VarBinds are returned, where

$\mathrm{N}$ equals g.non_repeaters,

$M$ equals g.max_repetitions, and

$\mathrm{R}$ is (number of SearchRanges in the GetBulk request) - $\mathrm{N}$.

The first $N$ SearchRanges are processed exactly as for the agentxGetNext-PDU.

If $M$ and $R$ are both non-zero, the remaining $R$ SearchRanges are processed iteratively to produce potentially many VarBinds. For each iteration $i$, such that $i$ is greater than zero and less than or equal 
to $\mathrm{M}$, and for each repeated SearchRange $\mathrm{s}$, such that $\mathrm{s}$ is greater than zero and less than or equal to $R$, the $(N+((i-1) * R)+s)-$ th $\operatorname{VarBind}$ is added to the agentx-Response-PDU as follows:

1) The subagent searches for a variable within the lexicographically ordered list of variable names for all variables it instantiates (without regard to registration of regions) within the indicated context and session, for which the following are all true:

- The variable's name is the (i)-th lexicographical successor to the $(\mathrm{N}+\mathrm{s})-\mathrm{th}$ requested OID.

(Note that if $i$ is 0 and the "include" field is 1 , the variable's name may be equivalent to, or the first lexicographical successor to, the $(\mathrm{N}+\mathrm{s})-\mathrm{th}$ requested OID.)

- If the ending OID is not null, the variable's name lexicographically precedes the ending OID.

If all of these conditions are met, $v$.name is set to the located variable's name. v.type and v.data are encoded to represent the variable's syntax and value, as described in section 5.4, "Value Representation".

2) If no such variable exists, the VarBind is set to' endOfMibView" as described in section 5.4, "Value Representation". V.name is set to V.name of the (N+( ( $\mathrm{i}-$ $2) * R)+s$ ) -th VarBind unless i is currently 1, in which case it is set to the value of the starting OID in the $(\mathrm{N}+\mathrm{s})$-th SearchRange.

Note that further iterative processing should stop if

- For any iteration i, all s values of v.type are ' endofMibView'.

- An Agentx transport constraint or other implementationspecific constraint is reached.

7.2.4. Subagent Processing of agentx-Testset, -Commitset, -UndoSet, -Cleanupset-PDUs

A conformant Agentx subagent must support the agentx-Test Set, -Commitset, -Undoset, and-Cleanupset PDUs, and must support multiple variables being supplied in the agentx-Testset-PDU. 
These four PDUs are used to collectively perform the indicated management operation. An agentx-Response-PDU is sent in reply to each of the PDUs (except-Cleanupset), to inform the master agent of the state of the operation.

The master agent must serialize set transactions for each session. That is, a session need not handle multiple concurrent set transactions.

These Response-PDUs do not contain a VarBindList.

\subsubsection{Subagent Processing of the agentx-TestSet-PDU}

Upon the subagent's receipt of an agentx-TestSet-PDU, each VarBind in the PDU is validated until they are all successful, or until one fails, as described in section 4.2.5 of RFC 1905 [13]. The subagent validates variables with respect to the context and session indicated in the testset-PDU.

If each VarBind is successful, the subagent has a further responsibility to ensure the availability of all resources (memory, write access, etc.) required for successfully carrying out a subsequent agentx-Commitset operation. If this cannot be guaranteed, the subagent should set res.error to 'resourceUnavailable'. As a result of this validation step, an agentx-Response-PDU is sent in reply whose res.error field is set to one of the following SNMPV2 PDU error-status values (see section 3, "Definitions", in RFC 1905 [13]):

$\begin{array}{lr}\text { noError } & (0), \\ \text { genErr } & (5), \\ \text { noAccess } & (6), \\ \text { wrongType } & (7), \\ \text { wrongLength } & (8), \\ \text { wrongEncoding } & (9), \\ \text { wrongValue } & (10), \\ \text { noCreation } & (11), \\ \text { inconsistentValue } & (12), \\ \text { resourceUnavailable } & (13), \\ \text { notWritable } & (17), \\ \text { inconsistentName } & (18)\end{array}$

If this value is not 'noError', the res.index field must be set to the index of the VarBind for which validation failed. 
Implementation of rigorous validation code may be one of the most demanding aspects of subagent development. Implementors are strongly encouraged to do this right, so as to avoid if at all possible the extensible agent's having to return 'commitFailed' or 'undoFailed' during subsequent processing.

7.2.4.2. Subagent Processing of the agentx-Commitset-PDU

The agentx-CommitSet-PDU indicates that the subagent should actually perform (as described in the post-validation sections of 4.2 .5 of RFC 1905 [13]) the management operation indicated by the previous TestSet-PDU. After carrying out the management operation, the subagent sends in reply an agentx-Response-PDU whose res.error field is set to one of the following SNMPV2 PDU error-status values (see section 3, "Definitions", in RFC 1905 [13]):

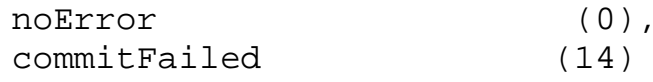

If this value is 'commitfailed', the res.index field must be set to the index of the VarBind (as it occurred in the agentx-TestSet-PDU) for which the operation failed. Otherwise res.index is set to 0 .

\subsubsection{Subagent Processing of the agentx-UndoSet-PDU}

The agentx-UndoSet-PDU indicates that the subagent should undo the management operation requested in a preceding Commitset-PDU. The undo process is as described in section 4.2.5 of RFC 1905 [13].

After carrying out the undo process, the subagent sends in reply an agentx-Response-PDU whose res.error field is set to one of the following SNMPv2 PDU error-status values (see section 3, "Definitions", in RFC 1905 [13]):
noError
undoFailed

If this value is 'undoFailed', the res.index field must be set to the index of the VarBind (as it occurred in the agentx-Testset-PDU) for which the operation failed. Otherwise res.index is set to 0 .

This PDU also signals the end of processing of the management operation initiated by the previous Testset-PDU. The subagent should release resources, etc. as described in section 7.2.4.4, "Subagent Processing of the agentx-Cleanupset-PDU". 
7.2.4.4. Subagent Processing of the agentx-Cleanupset-PDU

The agentx-Cleanupset-PDU signals the end of processing of the management operation requested in the previous Testset-PDU. This is an indication to the subagent that it may now release any resources it may have reserved in order to carry out the management request. No response is sent by the subagent.

\subsubsection{Master Agent Processing of Agentx Responses}

The master agent now marshals all subagent AgentX response PDUs and builds an SNMP response PDU. In the next several subsections, the initial processing of all subagent AgentX response PDUs is described, followed by descriptions of subsequent processing for each specific subagent Response.

\subsubsection{Common Processing of All AgentX Response PDUs}

1) If a response is not received on a session within the timeout interval for this dispatch, it is treated as if the subagent had returned 'genErr' and processed as described below.

A timeout may be due to a variety of reasons, and does not necessarily denote a failed or malfunctioning subagent. As such, the master agent's response to a subagent timeout is implementation-specific, but with the following constraint:

A session that times out on three consecutive Agentx requests is considered unable to respond, and the master agent must close the AgentX session as described in section 7.1.8, "Processing the agentx-Close-PDU", step (2).

2) Otherwise, the h.packetID, h.sessionID, and h.transactionID fields of the AgentX response PDU are used to correlate subagent responses. If the response does not pertain to this SNMP operation, it is ignored.

3) Otherwise, the responses are processed jointly to form the SNMP response PDU.

\subsubsection{Processing of Responses to agentx-Get-PDUs}

After common processing of the subagent's response to an agentx-GetPDU (see section 7.2.5.1, "Common Processing of All AgentX Response PDUs", above), processing continues with the following steps: 
1) For any received AgentX response PDU, if res.error is not 'noError', the SNMP response PDU's error code is set to this value. If res.error contains an Agentx specific value (e.g. 'parseError'), the SNMP response PDU's error code is set to a value of genErr instead. Also, the SNMP response PDU's error index is set to the index of the variable binding corresponding to the failed VarBind in the subagent's AgentX response PDU.

All other AgentX response PDUs received due to processing this SNMP request are ignored. Processing is complete; the SNMP Response PDU is ready to be sent (see section 7.2.6, "Sending the SNMP Response-PDU") .

2) Otherwise, the content of each VarBind in the Agentx response PDU is used to update the corresponding variable binding in the SNMP Response-PDU.

7.2.5.3. Processing of Responses to agentx-GetNext-PDU and agentx-GetBulk-PDU

After common processing of the subagent's response to an agentxGetNext-PDU or agentx-GetBulk-PDU (see section 7.2.5.1, "Common Processing of All AgentX Response PDUs", above), processing continues with the following steps:

1) For any received AgentX response PDU, if res.error is not 'noError', the SNMP response PDU's error code is set to this value. If res.error contains an Agentx specific value (e.g. 'parseError'), the SNMP response PDU's error code is set to a value of genErr instead. Also, the SNMP response PDU's error index is set to the index of the variable binding corresponding to the failed VarBind in the subagent's AgentX response PDU.

All other AgentX response PDUs received due to processing this SNMP request are ignored. Processing is complete; the SNMP response $\mathrm{PDU}$ is ready to be sent (see section 7.2.6, "Sending the SNMP Response-PDU") .

2) Otherwise, the content of each VarBind in the AgentX response PDU is used to update the corresponding VarBind in the SNMP response PDU.

After all expected Agentx response PDUs have been processed, if any VarBinds still contain the value 'endOfMibView' in their v.type fields, processing must continue: 
3) A new iteration of Agentx request dispatching is initiated (as described in section 7.2.1.2, "agentx-GetNext-PDU"), in which only those VarBinds whose v.type is 'endOfMibView' are processed.

4) For each such VarBind, an authoritative target MIB region is identified in which the master agent expects to find suitable MIB variables. The target session is the one on which this new target region was registered.

The starting OID in each SearchRange is set to the value of v.name for the corresponding VarBind, and its "include" field is set to 0 .

5) The value of transactionID must be identical to the value used during the previous iteration.

6) The AgentX PDUs are sent on the target session(s), and the responses are received and processed according to the steps described in section 7.2.5, "Master Agent Processing of AgentX Responses".

7) This process continues iteratively until a complete SNMP Response-PDU has been built, or until there remain no authoritative MIB regions to query.

Note that r.subtree for the new target region identified in step 4) may not lexicographically succeed r.subtree for the region that has returned 'endofMibView'. For example, consider the following registry:

$\begin{array}{llll}\text { session A } & \text { 'mib-2' } & (1.3 .6 .1 .2 .1) \\ \text { session B } & \text { 'ip' } & (1.3 .6 .1 .2 .1 .4) \\ \text { session C } & \text { 'tcp' } & (1.3 .6 .1 .2 .1 .6)\end{array}$

If while processing a GetNext-Request-PDU session B returns 'endOfMibView' for a variable name within 1.3.6.1.2.1.4, the target MIB region identified in step 4) would be 1.3.6.1.2.1 (since it may contain variables whose names precede 1.3.6.1.2.1.6).

Note also that if session A returned variables from within 1.3.6.1.2.1.6, they must be discarded since session A is NOT authoritative for that region.

7.2.5.4. Processing of Responses to agentx-Testset-PDUs

After common processing of the subagent's response to an agentxTestSet-PDU (see section 7.2.5.1, "Common Processing of All AgentX Response PDUs", above), processing continues with the further 
exchange of Agentx PDUs. The value of h.transactionid in the agentx-CommitSet, -UndoSet, and-CleanupSet-PDUs must be identical to the value sent in the testSet-PDU.

The state transitions and PDU sequences are depicted in section 7.3, "State Transitions".

The set of all sessions who have been sent an agentx-TestSet-PDU for this particular transaction are referred to below as "involved sessions".

1) If any target session's response is not 'noError', all other agentx-Response-PDUs received due to processing this SNMP request are ignored.

An agentx-CleanupSet-PDU is sent to all involved sessions. Processing is complete; the SNMP response PDU is constructed as described below in 7.2.6, "Sending the SNMP Response-PDU".

2) Otherwise an agentx-CommitSet-PDU is sent to all involved sessions.

7.2.5.5. Processing of Responses to agentx-Commitset-PDUs

After common processing of the subagent's response to an agentxCommitset-PDU (see section 7.2.5.1, "Common Processing of All AgentX Response PDUs", above), processing continues with the following steps:

1) If any response is not 'noError', the SNMP response PDU's error code is set to this value. If res.error contains an Agentx specific value (e.g. 'parseError'), the SNMP response PDU's error code is set to a value of genErr instead. Also, the SNMP response PDU's error index is set to the index of the VarBind corresponding to the failed VarBind in the agentx-Testset-PDU.

An agentx-UndoSet-PDU is sent to each target session that has been sent an agentx-CommitSet-PDU. An agentx-CleanupSet-PDU is sent to the remainder of the involved sessions.

2) Otherwise an agentx-Cleanupset-PDU is sent to all involved sessions. Processing is complete; the SNMP response PDU is constructed as described below in section 7.2.6, "Sending the SNMP Response-PDU". 
7.2.5.6. Processing of Responses to agentx-UndoSet-PDUs

After common processing of the subagent's response to an agentxUndoSet-PDU (see section 7.2.5.1, "Common Processing of All AgentX Response PDUs", above), processing continues with the following steps:

1) If any response is 'undoFailed' the SNMP response PDU's error code is set to this value. Also, the SNMP response PDU's error index is set to 0 .

2) Otherwise, if any response is not 'noError' the SNMP response PDU's error code is set to this value. Also, the SNMP response PDU's error index is set to the index of the VarBind corresponding to the failed VarBind in the agentx-Testset-PDU. If res.error is an AgentX specific value (e.g. 'parseError'), the SNMP response PDU's error code is set to a value of genErr instead.

3) Otherwise the SNMP response PDU's error code and error index were set in section 7.2 .5 .5 step 1)

\subsubsection{Sending the SNMP Response-PDU}

Once the processing described in section 7.2.5, "Master Agent Processing of Agentx Responses" is complete, there is an SNMP response PDU available. The master agent now implements the Elements of Procedure for the applicable version of the SNMP protocol in order to encapsulate the PDU into a message, and transmit it to the originator of the SNMP management request. Note that this may involve altering the PDU contents (for instance, to replace the original VarBinds if an error condition is to be returned).

The response PDU may also be altered in order to support the SNMPV1 PDU. In such cases the required PDU mapping is that defined in $R F C$ 2089 [25]. (Note in particular that the rules for handling Counter64 syntax may require re-sending Agentx GetBulk or GetNext PDUs until a VarBind of suitable syntax is returned.)

\subsubsection{MIB Views}

AgentX subagents are not aware of MIB views, since view information is not contained in AgentX PDUs.

As stated above, the descriptions of procedures in section 7, "Elements of Procedure", of this memo are not intended to constrain the internal architecture of any conformant implementation. In particular, the master agent procedures described in section 7.2.1, 
"Dispatching AgentX PDUs" and in section 7.2.5, "Master Agent Processing of Agentx Responses" may be altered so as to optimize AgentX exchanges when implementing MIB views.

Such optimizations are beyond the scope of this memo. But note that section 7.2.3, "Subagent Processing of agentx-Get, GetNext, GetBulkPDUs", defines subagent behavior in such a way that alteration of SearchRanges may be used in such optimizations.

7.3. State Transitions

State diagrams are presented from the master agent's perspective for transport connection and session establishment, and from the subagent's perspective for set transaction processing.

7.3.1. Set Transaction States

The following table presents, from the subagent's perspective, the state transitions involved in set transaction processing: 


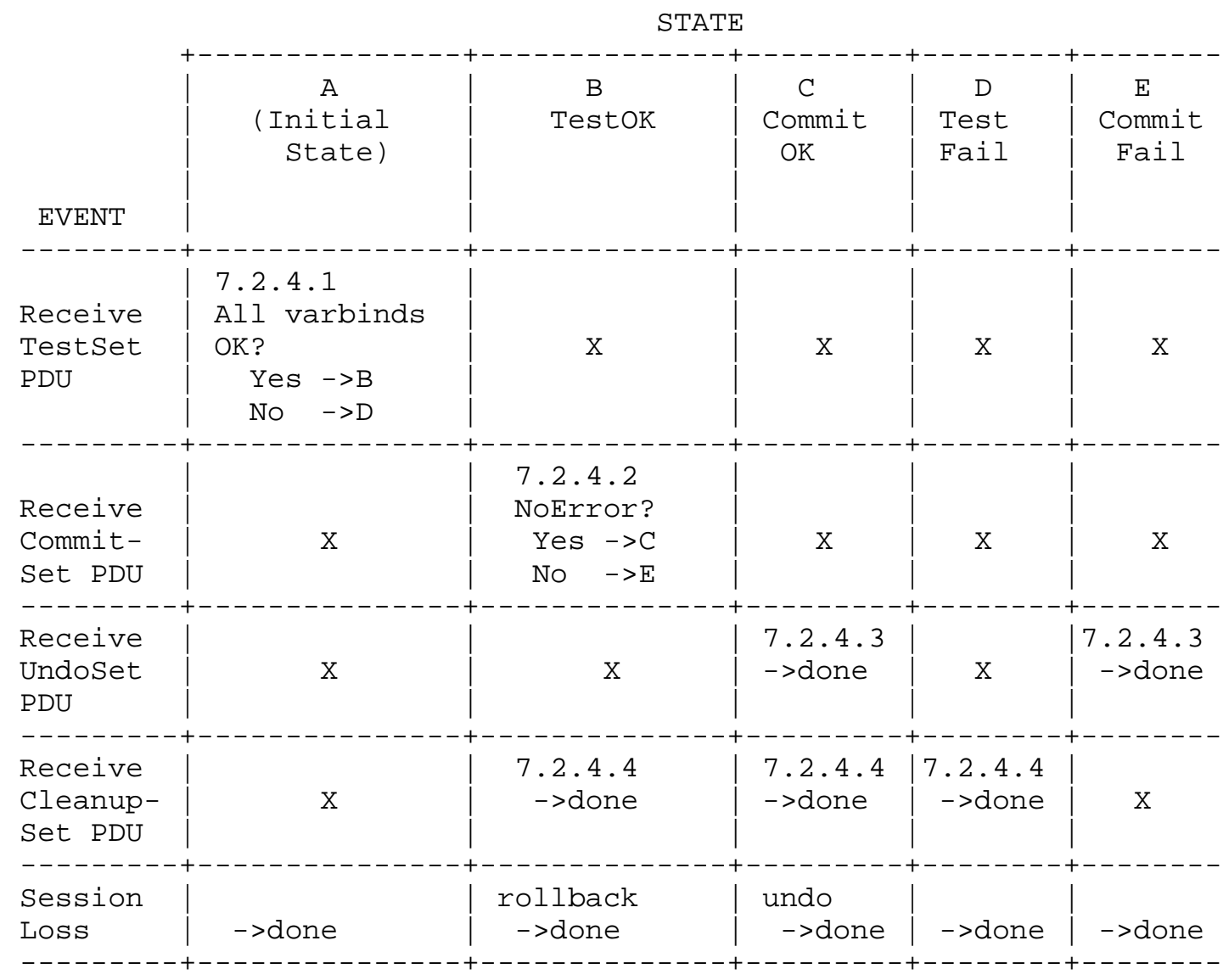

There are three possible sequences that a subagent may follow for a particular set transaction:

1) Test Set Commitset Cleanupset

2) Test Set Commitset UndoSet

3) Testset Cleanupset

Note that a single PDU sequence may result in multiple paths through the finite state machine (FSM). For example, the sequence

Test Set Commitset Undoset

may walk through either of these two state sequences:

(initial) TestoK CommitoK (done)

(initial) TestoK CommitFail (done) 


\subsubsection{Transport Connection States}

The following table presents, from the master agent's perspective, the state transitions involved in transport connection setup and teardown:

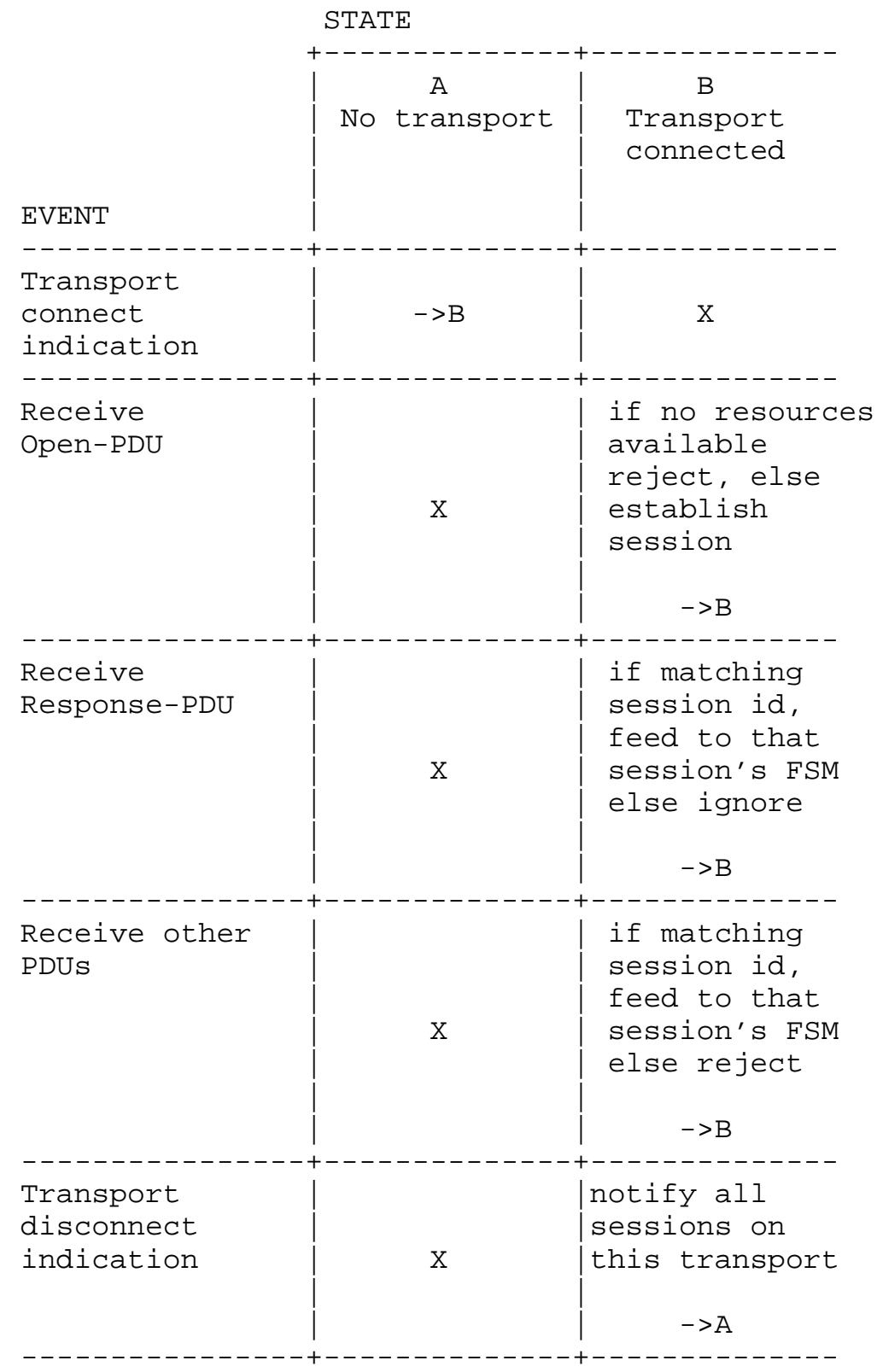




\subsubsection{Session States}

The following table presents, from the master agent's perspective, the state transitions involved in session setup and teardown:

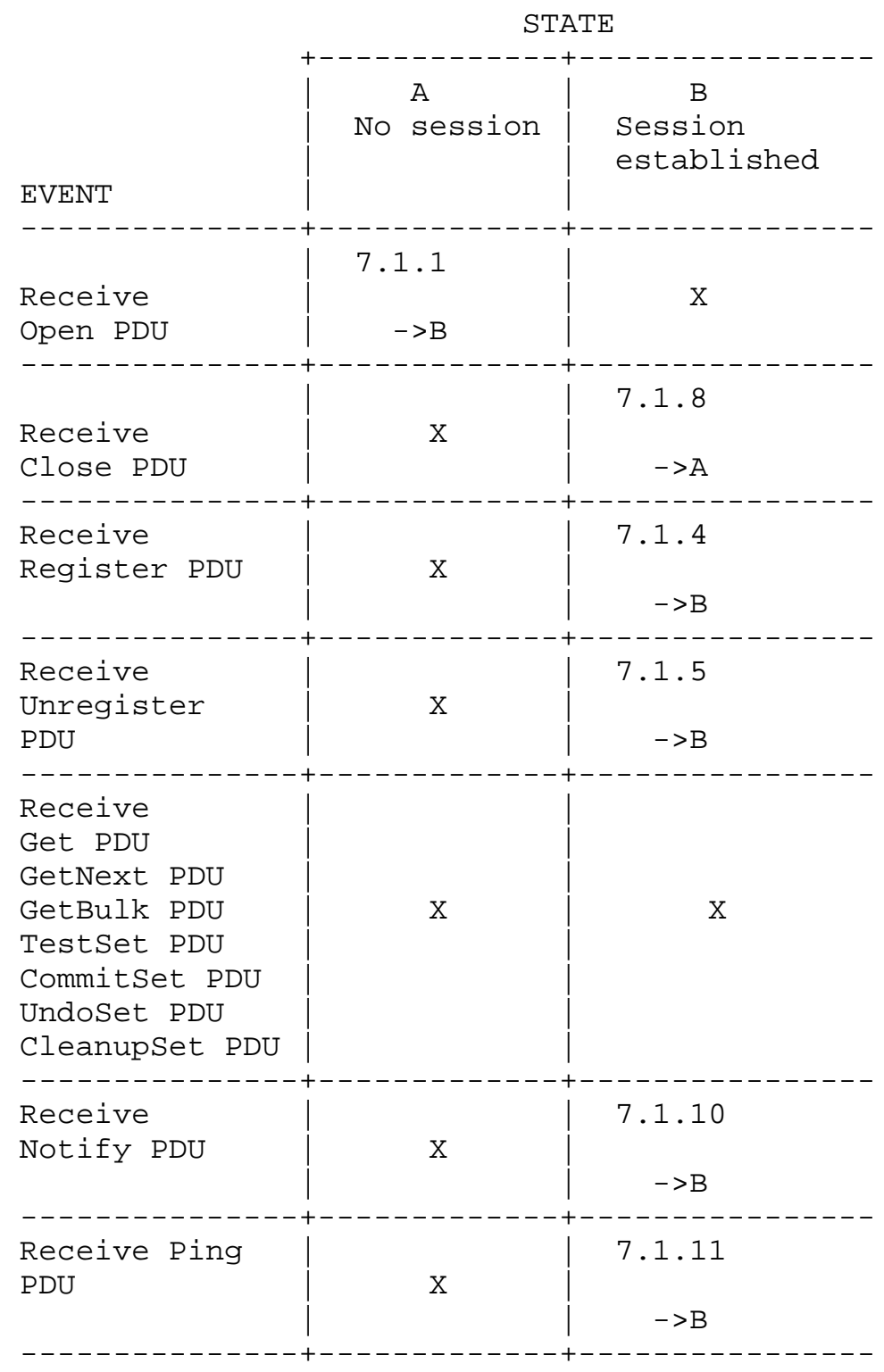

(continued next page) 


\begin{tabular}{|c|c|c|}
\hline $\begin{array}{l}\text { Receive } \\
\text { IndexAllocate } \\
\text { PDU }\end{array}$ & $\mathrm{X}$ & $\begin{array}{r}7.1 .2 \\
->B\end{array}$ \\
\hline $\begin{array}{l}\text { Receive } \\
\text { IndexDeallocate } \\
\text { PDU }\end{array}$ & $X$ & $\begin{array}{r}7.1 .3 \\
->B\end{array}$ \\
\hline $\begin{array}{l}\text { Receive } \\
\text { AddAgentxCaps } \\
\text { PDU }\end{array}$ & $X$ & $\begin{array}{r}7.1 .6 \\
->B\end{array}$ \\
\hline $\begin{array}{l}\text { Receive } \\
\text { RemoveAgentxCap } \\
\text { PDU }\end{array}$ & $X$ & $\begin{array}{r}7.1 .7 \\
->B\end{array}$ \\
\hline $\begin{array}{l}\text { Receive } \\
\text { Response PDU }\end{array}$ & $X$ & $\begin{array}{r}7.2 .5 \\
->B\end{array}$ \\
\hline $\begin{array}{l}\text { Receive } \\
\text { Other PDU }\end{array}$ & X & $\mathrm{X}$ \\
\hline
\end{tabular}

8. Transport Mappings

The same Agentx PDU formats, encodings, and elements of procedure are used regardless of the underlying transport.

\subsection{AgentX over TCP}

\subsubsection{Well-known Values}

The master agent accepts TCP connection requests for the well-known port 705. Subagents connect to the master agent using this port number.

\subsubsection{Operation}

Once a TCP connection has been established, the Agentx peers use this connection to carry all Agentx PDUs. Multiple Agentx sessions may be established using the same TCP connection. AgentX PDUs are sent within an Agentx session. Agentx peers are responsible for mapping the h.sessionID to a particular TCP connection. 
The Agentx entity must not "interleave" AgentX PDUs within the TCP byte stream. All the bytes of one PDU must be sent before any bytes of a different PDU. The receiving entity must be prepared for TCP to deliver byte sequences that do not coincide with Agentx PDU boundaries.

\subsection{AgentX over UNIX-domain Sockets}

Many (BSD-derived) implementations of the UNIX operating system support the UNIX pathname address family (AF_UNIX) for socket communications. This provides a convenient method of sending and receiving data between processes on the same host.

Mapping Agentx to this transport is useful for environments that

- wish to guarantee subagents are running on the same managed node as the master agent, and where

- sockets provide better performance than TCP or UDP, especially in the presence of heavy network I/O

\subsubsection{Well-known Values}

The master agent creates a well-known UNIX-domain socket endpoint called "/var/agentx/master". (It may create other, implementationspecific endpoints.)

This endpoint name uses the character set encoding native to the managed node, and represents a UNIX-domain stream (SOCK_STREAM) socket.

\subsubsection{Operation}

Once a connection has been established, the Agentx peers use this connection to carry all Agentx PDUs.

Multiple Agentx sessions may be established using the same connection. AgentX PDUs are sent within an AgentX session. AgentX peers are responsible for mapping the h.sessionID to a particular connection.

The Agentx entity must not "interleave" Agentx PDUs within the socket byte stream. All the bytes of one PDU must be sent before any bytes of a different PDU. The receiving entity must be prepared for the socket to deliver byte sequences that do not coincide with AgentX PDU boundaries. 


\section{Security Considerations}

This memo defines a protocol between two processing entities, one of which (the master agent) is assumed to perform authentication of received SNMP requests and to control access to management information. The master agent performs these security operations independently of the other processing entity (the subagent).

Security considerations require three questions to be answered:

1. Is a particular subagent allowed to initiate a session with a particular master agent?

2. During an Agentx session, is any SNMP security-related information (for example, community names) passed from the master agent to the subagent?

3. During an AgentX session, what part of the MIB tree is this subagent allowed to register?

The answer to the third question is: A subagent can register any subtree (subject to Agentx elements of procedure, section 7.1.4, "Processing the agentx-Register-PDU"). Currently there is no access control mechanism defined in Agentx. A concern here is that a malicious subagent that registers an unauthorized "sensitive" subtree, could see modification requests to those objects, or by giving its own clever answer to NMS queries, could cause the NMS to do something that leads to information disclosure or other damage.

The answer to the second question is: No.

Now we can answer the first question. Agentx does not contain a mechanism for authorizing/refusing session initiations. Thus, controlling subagent access to the master agent may only be done at a lower layer (e.g., transport).

An Agentx subagent can connect to a master agent using either a network transport mechanism (e.g., TCP), or a "local" mechanism (e.g., shared memory, named pipes).

In the case where a local transport mechanism is used and both subagent and master agent are running on the same host, connection authorization can be delegated to the operating system features. The answer to the first security question then becomes: "If and only if the subagent has sufficient privileges, then the operating system will allow the connection". 
If a network transport is used, currently there is no inherent security. Transport Layer Security, SSL, or IPsec SHOULD be used to control and protect subagent connections in this mode of operation.

However, we RECOMMEND that subagents always run on the same host as the master agent and that operating system features be used to ensure that only properly authorized subagents can establish connections to the master agent.

10. Acknowledgements

The initial development of this memo was heavily influenced by the DPI 2.0 specification RFC 1592 [26].

This document was produced by the IETF Agent Extensibility (AgentX) Working Group, and benefited especially from the contributions of the following working group members:

David Battle, Uri Blumenthal, Jeff Case, Maria Greene, Lauren Heintz, Dave Keeney, Harmen van der Linde, Bob Natale, Aleksey Romanov, Don Ryan, and Juergen Schoenwaelder.

An honorable mention is extended to Randy Presuhn in recognition for his numerous technical contributions to this specification; for his many answers provided on (and hosting of) the Agentx e-mail list and ftp site, and, for the valued support and guidance Randy provided to the Working Group chair.

The Agentx Working Group is chaired by:

Bob Natale

$\mathrm{ACE} * \mathrm{COMM}$ Corporation

704 Quince Orchard Road

Gaithersburg, MD 20878

Phone: +1-301-721-3000

Fax: +1-301-721-3001

EMail: bnatale@acecomm.com 
11. Authors' and Editor's Addresses

Mike Daniele

Compaq Computer Corporation

110 Spit Brook Rd

Nashua, NH 03062

Phone: +1-603-881-1423

EMail: daniele@zk3.dec.com

Bert Wijnen

IBM T.J.Watson Research

Schagen 33

3461 GL Linschoten

Netherlands

Phone: +31-348-432-794

EMail: wijnen@vnet.ibm.com

Mark Ellison (WG editor)

Ellison Software Consulting, Inc.

38 Salem Road

Atkinson, $\mathrm{NH} 03811$

Phone: +1-603-362-9270

EMail: ellison@world.std.com

Dale Francisco (editor)

Cisco systems

150 Castilian Dr

Goleta CA 93117

Phone: +1-805-961-3642

Fax: $\quad+1-805-961-3600$

EMail: dfrancisdcisco.com 


\section{References}

[1] Harrington, D., Presuhn, R. and B. Wijnen, "An Architecture for Describing SNMP Management Frameworks", RFC 2571, April 1999.

[2] Rose, M. and K. McCloghrie, "Structure and Identification of Management Information for TCP/IP-based Internets", STD 16, RFC 1155, May 1990 .

[3] Rose, M. and K. McCloghrie, "Concise MIB Definitions", STD 16, RFC 1212, March 1991.

[4] Rose, M., "A Convention for Defining Traps for use with the SNMP", RFC 1215, March 1991.

[5] McCloghrie, K., Perkins, D., Schoenwaelder, J., Case, J., Rose, M. and S. Waldbusser, "Structure of Management Information Version 2 (SMIv2)", STD 58, RFC 2578, April 1999.

[6] McCloghrie, K., Perkins, D., Schoenwaelder, J., Case, J., Rose, M. and S. Waldbusser, "Textual Conventions for SMIv2", STD 58, RFC 2579, April 1999.

[7] McCloghrie, K., Perkins, D., Schoenwaelder, J., Case, J., Rose, M. and S. Waldbusser, "Conformance Statements for SMIv2", STD 58, RFC 2580, April 1999.

[8] Case, J., Fedor, M., Schoffstall, M. and J. Davin, "Simple Network Management Protocol", STD 15, RFC 1157, May 1990.

[9] Case, J., McCloghrie, K., Rose, M. and S. Waldbusser, "Introduction to Community-based SNMPv2", RFC 1901, January 1996.

[10] Case, J., McCloghrie, K., Rose, M. and S. Waldbusser, "Transport Mappings for Version 2 of the Simple Network Management Protocol (SNMPv2)", RFC 1906, January 1996.

[11] Case, J., Harrington D., Presuhn R. and B. Wijnen, "Message Processing and Dispatching for the Simple Network Management Protocol (SNMP)", RFC 2572, April 1999.

[12] Blumenthal, U. and B. Wijnen, "User-based Security Model (USM) for version 3 of the Simple Network Management Protocol (SNMPv3)", RFC 2574, April 1999. 
[13] Case, J., McCloghrie, K., Rose, M. and S. Waldbusser, "Protocol Operations for Version 2 of the Simple Network Management Protocol (SNMPv2)", RFC 1905, January 1996.

[14] Levi, D., Meyer, P. and B. Stewart, "SNMPv3 Applications", RFC 2573, April 1999.

[15] Wijnen, B., Presuhn, R. and K. McCloghrie, "View-based Access Control Model (VACM) for the Simple Network Management Protocol (SNMP) ", RFC 2575, April 1999.

[16] Case, J., Mundy, R., Partain, D. and B. Stewart, "Introduction to Version 3 of the Internet-standard Network Management Framework", RFC 2570, April 1999.

[17] Case, J., McCloghrie, K., Rose, M. and S. Waldbusser, "Management Information Base for Version 2 of the Simple Network Management Protocol (SNMPv2)", RFC 1907, January 1996.

[18] Information processing systems - Open systems Interconnection Specification of Abstract Syntax Notation One (ASN.1), International Organization for Standardization. International Standard 8824, (December, 1987).

[19] McCloghrie, K. and F. Kastenholz, "The Interfaces Group MIB using SMIv2", RFC 2233, November 1997.

[20] Case, J., "FDDI Management Information Base", RFC 1285, January 1992 .

[21] Krupczak, C. and J. Saperia, "Definitions of System-Level Managed Objects for Applications", RFC 2287, April 1997.

[22] Kalbfleisch, C., Krupczak, C., Presuhn, R. and J. Saperia, "Application Management MIB", RFC 2564, May 1999.

[23] Reynolds, J. and J. Postel, "Assigned Numbers", STD 2, RFC 1700, October 1994 .

[24] Case, J., McCloghrie, K., Rose, M. and S. Waldbusser, "Coexistence between Version 1 and Version 2 of the Internetstandard Network Management Framework", RFC 1908, January 1996.

[25] Wijnen, B. and D. Levi, "V2ToV1: Mapping SNMPv2 onto SNMPv1 Within a Bilingual SNMP Agent", RFC 2089, January 1997. 
[26] Wijnen, B., Carpenter, G., Curran, K., Sehgal, A. and G. Waters, "Simple Network Management Protocol: Distributed Protocol Interface, Version 2.0", RFC 1592, March 1994.

[27] Bradner, S., "Key words for use in RFCs to Indicate Requirement Levels", BCP 14, RFC 2119, March 1997.

13. Notices

The IETF takes no position regarding the validity or scope of any intellectual property or other rights that might be claimed to pertain to the implementation or use of the technology described in this document or the extent to which any license under such rights might or might not be available; neither does it represent that it has made any effort to identify any such rights. Information on the IETF's procedures with respect to rights in standards-track and standards-related documentation can be found in BCP-11. Copies of claims of rights made available for publication and any assurances of licenses to be made available, or the result of an attempt made to obtain a general license or permission for the use of such proprietary rights by implementors or users of this specification can be obtained from the IETF Secretariat.

The IETF invites any interested party to bring to its attention any copyrights, patents or patent applications, or other proprietary rights which may cover technology that may be required to practice this standard. Please address the information to the IETF Executive Director. 
A. Changes relative to RFC 2257

Changes on the wire:

- The agentx-Notify-PDU and agentx-Close-PDU now generate an agentx-Response-PDU.

- The res.error field may contain three new error codes: parseFailed(266), requestDenied(267), and processingError(268).

Clarifications to the text of the memo:

- Modified the text of step (4) in section 4.2, "Applicability" to separate the two concerns of row creation, and counters that count rows.

- The use of the r.range_subid field is more clearly defined in section 6.2.3, "The agentx-Register-PDU".

- Default priority (127) for registration added to the description of r.priority in section 6.2.3, "The agentxRegister-PDU" .

- Made the distinction of "administrative processing" PDUs and "SNMP request processing" PDUs in section 6.1, "AgentX PDU Header" description of h.type. This distinction is used in the Elements of Procedure relative to the res.sysuptime and res.error fields.

- Rewrote portions of text in section 6.2.3, "The agentxRegister-PDU" to be more explicit about the following points:

- There is a default registration priority of 127 .

- Improved the description of r.range_subid, independent of the prefix in r.region.

- Improved description and examples of how to use the registration mechanism.

- Added a description for r.upper_bound.

- changed r.region to r.subtree (because the text used the terms "region", "range", and "OID range" in too loose a fashion. r.subtree can not represent anything more by itself than a simple subtree. In conjunction with r.range_subid and r.upper_bound, it can represent a "region", that is, a union of subtrees)

- Modified the text in section 6.2.4, "The agentx-Unregister-PDU" to include a description of u.range_subid and u.upper_bound 
- Added use of the 'requestDenied' error code in section 7.1.4, "Processing the agentx-Register-PDU".

- Removed text in section 7, "Elements of Procedure" on parse errors and protocol errors.

- Added a new section, 7.1, "Processing Agentx Administrative Messages" which defines common processing and how to use the 'parseError' and 'processingError' instead of closing a session, and how to handle context.

- Removed the common processing text from the other administrative processing Elements of Procedure sections, and added a reference to section 7.1, "Processing AgentX Administrative Messages". The affected sections are:

$$
\begin{aligned}
& \text { - 7.1.2, "Processing the agentx-IndexAllocate-PDU" } \\
& \text { - 7.1.3, "Processing the agentx-IndexDeallocate-PDU" } \\
& \text { - 7.1.4, "Processing the agentx-Register-PDU" } \\
& \text { - 7.1.5, "Processing the agentx-Unregister-PDU" } \\
& \text { - 7.1.6, "Processing the agentx-AddAgentCaps-PDU" } \\
& \text { - 7.1.7, "Processing the agentx-RemoveAgentCaps-PDU" } \\
& \text { - 7.1.8, "Processing the agentx-Close-PDU" } \\
& \text { - 7.1.10, "Processing the agentx-Notify-PDU" } \\
& \text { - 7.1.11, "Processing the agentx-Ping-PDU" }
\end{aligned}
$$

- Reworked the text in section 7.1.1, "Processing the agentx-Open-PDU" to include new error codes, and, to eliminate reference to an indicated context.

- Modified the text in Section 7.1.10, "Processing the agentx-Notify-PDU" to state that context checking is performed.

- Substantially modified the text in section 7.1.4.1, "Handling Duplicate and Overlapping Subtrees".

- Removed the section on "Using the agentx-IndexAllocate-PDU" and added section 7.1.4.2, "Registering Stuff". This change is intended to provide a more concise and a more cohesive description of how things are supposed to work.

- Modified the test in section 7.1.5, "Processing the agentx-Unregister-PDU" to require a match on u.range_subid and on u.upper_bound when these fields were applicable in the corresponding agentx-Register-PDU. 
- Removed all references to "splitting", and all uses of the term "OID range". The text now refers to regions or subtrees directly, and relies on rule (1), "Honoring the Registry", in section 7.2.1, "Dispatching AgentX PDUs".

- Modified text in clause 4(c) of section 7.2.1, "Dispatching AgentX PDUs", clarifying that the master agent can use its implementation-specific default timeout value when the timeout value registered by the subagent is impractical.

- Added text in section 7.2.2, "Subagent Processing" describing common processing.

- Added an example to the text in section 7.2.5.3, "Processing of Responses to agentx-GetNext-PDU and agentx-GetBulk-PDU", and, removed the definition of "contains" from this section.

- Modified text in step (1) of section 7.2.5.5, "Processing of Responses to agentx-Commitset-PDUs", eliminating directive for master agent to ignore additional responses to agentx-Commitset-PDUs after the first error response.

- Modified text in section 7.2.5.6, "Processing of Responses to agentx-Undoset-PDUs", cleaning up commit/undo elements of procedure per feedback received on the Agentx email list.

- Modified the text in section 8.1.2, "Operation" to explicitly prohibit interleaved sends, and, added a caution about exchanging AgentX messages via TCP.

- Modified text to be more explicit that the OID in the agentx-Allocate-PDU is an OBJECT-TYPE and does not contain any instance sub-identifiers.

- Replaced the term "subagent" with the term "session" in many places throughout the text.

- Modified the text relative to master agent processing of the agentx-TestSet-PDU, agentx-CommitSet-PDU, and the agentx-UndoSet-PDU to explicitly state that only "involved" sessions receive an agentx-Commitset-PDU, and possibly, an agentx-Undoset-PDU.

- Modified the text to use the term "transaction", instead of "packet" (and others), where appropriate. This helps distinguish the overall transaction from a particular sequence of packets or PDUs. 
- Modified the text to explicitly state that a session is not required to support concurrent sets.

- Added section 13, "Notices".

- Added text to section 1, Introduction, relative to BCP 14 key words.

- Modified text to section 9, Security Considerations, to include use of BCP 14 key words.

- Modified text to section 9, Security Considerations, to include IPSEC as a suggested Transport Layer Security. 
Full Copyright statement

Copyright (C) The Internet Society (2000). All Rights Reserved.

This document and translations of it may be copied and furnished to others, and derivative works that comment on or otherwise explain it or assist in its implementation may be prepared, copied, published and distributed, in whole or in part, without restriction of any kind, provided that the above copyright notice and this paragraph are included on all such copies and derivative works. However, this document itself may not be modified in any way, such as by removing the copyright notice or references to the Internet society or other Internet organizations, except as needed for the purpose of developing Internet standards in which case the procedures for copyrights defined in the Internet Standards process must be followed, or as required to translate it into languages other than English.

The limited permissions granted above are perpetual and will not be revoked by the Internet society or its successors or assigns.

This document and the information contained herein is provided on an "AS IS" basis and THE INTERNET SOCIETY AND THE INTERNET ENGINEERING TASK FORCE DISCLAIMS ALL WARRANTIES, EXPRESS OR IMPLIED, INCLUDING BUT NOT LIMITED TO ANY WARRANTY THAT THE USE OF THE INFORMATION HEREIN WILL NOT INFRINGE ANY RIGHTS OR ANY IMPLIED WARRANTIES OF MERCHANTABILITY OR FITNESS FOR A PARTICULAR PURPOSE.

Acknowledgement

Funding for the RFC Editor function is currently provided by the Internet society. 